\title{
Analysis on Lie groups
}

Nick Th. Varopoulos

Dedicated to the memory of my mother

\section{Introduction.}

\subsection{Statement of the theorems.}

In what follows $G$ will denote a real connected Lie group and $\Delta=$ $-\sum_{j=1}^{n} X_{j}^{2}+X_{0}$ will denote some subelliptic left invariant Laplacian (cf. [1]). This, for us here, will mean that $X_{0}, X_{1}, \ldots, X_{n}$ are left invariant fields on $G$ (i.e. $X f_{g}=(X f)_{g}, f_{g}(x)=f(g x)$ ) and that $X_{1}, \ldots, X_{n}$ are generators of the Lie algebra of $G$ (i.e. together with all their successive brackets they span the Lie algebra of $G(c f .[2]))$. I shall denote by $d g=d^{\ell} g$ the left Haar measure of $G$ and by $d^{r} g=$ $d^{\ell}\left(g^{-1}\right)=m(g) d^{\ell} g$ the right Haar measure and by $m(g)=m_{G}(g)$ the modular function.

We can then construct $T_{t}=e^{-t \Delta}(t>0)$ the Heat diffusion semigroup and $\phi_{t}(g)$ the corresponding Heat diffusion kernel that is defined by

$$
T_{t} f(x)=\int_{G} f(y) \phi_{t}\left(y^{-1} x\right) d y, \quad t>0, x \in G, f \in C_{0}^{\infty}(G) .
$$

When $X_{0}=0$ we say that $\Delta=\Delta_{0}$ is driftless. A driftless Laplacian $\Delta_{0}$ is formally self adjoint with respect to $d^{r} g$. It follows that the modified Laplacian $\tilde{\Delta}=m^{1 / 2} \Delta_{0} m^{-1 / 2}$ is formally self adjoint with respect to $d g$. It is then more convenient to consider the modified semigroup $\tilde{T}_{t}=m^{1 / 2} e^{-t \Delta_{0}} m^{-1 / 2}$ and it is very easy to see (cf. [3]) that the 
$L^{2}\left(G ; d^{r} g\right) \rightarrow L^{2}\left(G ; d^{r} g\right)$ norm of the operator $T_{t}=e^{-t \Delta_{0}}$ (which is the same as the $L^{2}(G ; d g) \rightarrow L^{2}(G ; d g)$ norm of $\left.\tilde{T}_{t}\right)$ satisfies

$$
\left\|T_{t}\right\|_{2 \rightarrow 2}=e^{-\lambda t}
$$

where $\lambda$ is the spectral gap of $\Delta_{0}$ defined by:

$$
\lambda=\inf \left\{\int_{G}|\nabla f|^{2} d^{r} g: \int_{G} f^{2} d^{r} g=1\right\},
$$

where $|\nabla f|^{2}=\sum_{j=1}^{n}\left|X_{j} f\right|^{2}$.

In Chapter 1 of this paper we shall give an algebraic classification of $\mathfrak{g}$, the Lie algebra of $\mathrm{G}$, into two classes: the B-algebras and the NB-algebras. We say of course that $G$ is a B- (respectively NB-) group if $\mathfrak{g}$ is a B-(respectively NB-) algebra. We shall refer the reader to Chapter 1 for the precise definition that is algebraically very natural but fairly long to explain. In general terms one considers the minimal parabolic subgroups $P$ (cf. [26] for the definition of these subgroups when $G$ is semisimple. Here we extend the notion to general Lie groups by considering "maximal amenable subgroups" or rather a special class of such subgroups). One then considers the corresponding dynamical system $\operatorname{Ad}(P)$ and the classification amounts to the "hyperbolicity" or not of that system. If we denote, here and throughout, by $e \in G$ the neutral element of $G$ we have

Theorem $A$. Let $G$ be a Lie group as above and let $\Delta_{0}$ be a driftless Laplacian, let further $\phi_{t} \in C^{\infty}(G), \lambda \geq 0$ be the corresponding heat diffusion kernel and spectral gap respectively. Then

$\mathrm{A}_{1}$ ) If we assume that $G$ is a B-group then there exists $C, c_{1}$, $c_{2}>0$ such that

$$
\begin{aligned}
C^{-1} \exp \left(-\lambda t-c_{2} t^{1 / 3}\right) & \leq \phi_{t}(e) \\
& \leq C \exp \left(-\lambda t-c_{1} t^{1 / 3}\right), \quad t \geq 1 .
\end{aligned}
$$

$\mathrm{A}_{2}$ ) If we assume that $G$ is an NB-group then there exists $C>0$, $\nu \geq 0$ such that

$$
C^{-1} t^{-\nu} e^{-\lambda t} \leq \phi_{t}(e) \leq C t^{-\nu} e^{-\lambda t}, \quad t \geq 1
$$


By the standard local Harnack estimate (cf. [1], [4]) we can of course replace $\phi_{t}(e)$ by $\phi_{t}(g)(g \in G)$ but then the constant $C=C(g)>$ 0 depends on $g$.

Observe that the upper estimate $(0.2)$ with $\nu=0$ is very easy ( $c f$. [3]). The proof that the same index $\nu \geq 0$ can be used for both the upper and the lower estimate in $A_{2}$ ) is very technical. This will be done elsewhere. Here we shall show that some $\nu \geq 0$ exists for which the lower estimate in $(0.2)$ holds.

Another way to write the Heat diffusion semigroup is $T_{t} f=f * \mu_{t}$ where $d \mu_{t}(g)=\phi_{t}(g) d^{r} g$ is a probability measure on $G$ that in addition has a number of properties that qualifies it to be a "Gaussian" (Gs in short!) measure on $G$ in a sense that we shall make precise in Chapter 3. For any bounded measure $\mu$ on $G$, I shall denote by $\|\mu\|_{2 \rightarrow 2}$ the $L^{2}\left(G ; d^{r} g\right) \rightarrow L^{2}\left(G ; d^{r} g\right)$ norm of the operator $f \rightarrow f * \mu$. We have then

Theorem B. Let $G$ be a B-group as above and let $\mu \in \mathbb{P}(G)$ be a Gs-probability measure on $G$. Then there exists $c>0$ such that for all $\varphi \in C_{0}^{\infty}(G)$ we have

$$
\left\langle\varphi, \mu^{* n}\right\rangle=O\left(\left\|\mu^{n}\right\|_{2 \rightarrow 2} e^{-c n^{1 / 3}}\right) .
$$

The above clearly ( $c f .[1])$ contains the upper estimates of $(0.1)$. It is easy to see that in the estimate (0.3) we can replace $\left\|\mu^{n}\right\|_{2 \rightarrow 2}$ by $\sigma(\mu)^{n}$ where $\sigma(\mu) \geq 0$, the spectral radious of $\mu$ (i.e. the spectral radious of the operator $f \rightarrow f * \mu)$. The above theorem also holds if we replace the Gs-measure $\mu \in \mathbb{P}(G)$ by some measure that is compactly supported and has continuous (or even just $L^{2}$ ) density. The proof is but an easy modification of the one given in this paper and is if anything easier. The details will however not be given here. Observe finally that for symmetric measures we can easily adapt our methods to give lower estimates in Theorem $B$ that are in the same spirit as Theorem $A$.

\subsection{Guide to the paper.}

Chapter 1 is pure algebra and it presents some independent interest. Chapter 2 analyzes the geometry of Lie groups and it shows how the spectral gap can be "isolated" from the rest of the decay of the 
Heat kernel. Both the above sections are basic and are likely to play an important role in further developments. Chapter 3 is technical and is only one among many possible approachs to carry out the details of the proof of the upper estimates. The proof of the upper estimate is completed in chapter 4 where a fair amount of global structure theory of Lie group is needed. Observe however that for these upper estimates one needs chapter 2 only up to Section 2.4 and one needs very little algebra (essentially only the definitions of B-groups). A good way for the reader to start with this paper seems to me therefore to go streight for that upper estimate in chapter 4 and refer back to chapter 1, chapter 2 as needed. For the upper estimate one also needs Section B in the appendix.

Almost all of the algebra and the more intricate parts of chapter 2 are only needed for the lower estimates. In the proof of the lower estimate of (0.2) one more (rather unexpected) difficulty arises. The proof as I give it here is considerably easier if $\Delta$ is elliptic. The complications that arise when $\Delta$ is only subelliptic are quite formidable. This distinction disappears in the alternative, much more sophisticate (at the potential theoretic level), approach that will be used to show that the same $\nu$ can be used for the upper and lower estimate at (0.2). This approach will be presented elsewhere. My advise to the reader is therefore to ignore that difficulty and pretend, at least in a first reading, that $\Delta$ is elliptic.

The role of the appendix is crucial since it contains all the probability and potential theory that is needed in the rest of the paper. The appendix can (and should) be studied independently, and it has its own independent "guide to the reader" where I explain in particular what is needed for what. Whether it was a good idea to separate the material in this way is of course debatable. One thing is certain, this paper is very long and putting the appendix apart made my life a little easier.

\section{Chapter OV. An overview.}

The aim of this chapter (which properly speaking is not part of the paper since it was written after the rest of the writting was completed) is to give to the reader an overview of the subject that is developed in this paper as well as in some of my previous work in the area.

The material is presented here in general terms and with an emphasis on ideas and on the "intuitive picture". The price that one pays 
for this is in the precision or even in the accuracy in the presentation. I warn the reader that many assertions made in this chapter are, as such, incorrect. The "deviation" from what is actually correct however can be controled and one can say that the aim of this work is to make these ideas into real mathematics.

The only thing that a non expert needs for the reading of this chapter is the definition of a semidirect product $A<B$ of two groups (cf. [6]) and to have some idea of what a random walk and brownian motion is (cf. [36]). In reading the first two sections of this chapter the reader could also profit from [37], [38] (in [37] some explanation is offered for the missing page in [38]).

\section{OV.1. The $a x+b$ group.}

Let $G$ be the (only non abelian) two dimensional Lie group of affine transformations on $\mathbb{R}, \sigma: x \mapsto a x+b(x \in \mathbb{R})$ with $0<a=e^{\alpha} \in \mathbb{R}_{+}^{*}$ and $\alpha, b \in \mathbb{R}$. This group is the semidirect product $\mathbb{R}<\mathbb{R}_{+}^{*}$ since

$$
\sigma_{1} \sigma_{2}: x \longmapsto a_{1} a_{2} x+b_{2} a_{1}+b_{1}
$$

where the action of $\mathbb{R}_{+}^{*}$ on $\mathbb{R}$ is $b \mapsto b a$.

Let us now consider two probability distributions $\mu^{*} \in \mathbb{P}\left(\mathbb{R}_{+}^{*}\right)$, $\mu \in \mathbb{P}(\mathbb{R})$ and let $\nu=\mu \times \mu^{*}$ be the "product" measure that we obtain on $G$ by putting $\mu$ on $\mathbb{R}$ and $\mu^{*}$ on $\mathbb{R}_{+}^{*}$.

The beginning of the present work was when several years ago I observed that one could represent the random walk on $G$ generated by $\nu$ (alternatively the convolution powers $\nu^{* n}$ ) in a very simple and managable way. This idea I shall explain in this section.

Let $\left[g_{n}=\left(x_{n}, s_{n}^{*}\right) \in G: n \geq 1\right]$ be the paths of that random walks which formally is defined by $\mathbb{P}\left[g_{n+1} \in d x: g_{n}=y\right]=d \nu\left(y^{-1} x\right)$. By projecting $G \rightarrow \mathbb{R}_{+}^{*}$ we see that $s_{n}^{*}=x_{1}^{*} \cdots x_{n}^{*}$ performs a (multiplicative) random walk on $\mathbb{R}_{+}^{*}(\cong \mathbb{R})$ with transition probability $\mu^{*}$. The motion of $x_{n} \in \mathbb{R}$ does not, on the other hand, obey a simple stochastic law and there lies the difficulty of the problem.

The key observation is that once we "fix" (i.e. condition in formal probabilistic terms) the path $\omega=\left(s_{1}^{*}, s_{2}^{*}, \ldots\right)$ of the random walk on $\mathbb{R}_{+}^{*}$ then the motion $x_{1}, x_{2}, \cdots \in \mathbb{R}$ also becomes Markovian. The Markov process that we thus obtain is time inhomogeneous and we have

$$
\mathbb{P}\left[x_{n+1} \in d x / / x_{n}=y ; \omega\right]=d \mu_{n}(x-y),
$$


where $\mu_{n}$ is the measure on $\mathbb{R}$ that is obtained from $\mu$ after the dilatation $x \mapsto s_{n}^{*} x(x \in \mathbb{R})$.

This idea, simple though it is, goes a long way. Let us for simplicity make the assumption that $\mu \in \mathcal{N}(0,1)$ is a normal (i.e. Gaussian) variable (mean zero and covariance 1 ). Then $\mu_{n} \in \mathcal{N}\left(0, s_{n}^{*}\right)$. From this we can easily estimate the return probability of our random walk

$$
p(n)=\mathbb{P}\left[g_{n} \in\left[-b_{0}, b_{0}\right]<\left[a_{0}^{-1}, a_{0}\right] \subset G\right]
$$

for some fixed $0<b_{0} \in \mathbb{R}, a_{0} \in \mathbb{R}_{+}^{*}$. The first step is to estimate the return probability of the conditioned random walk (i.e. fixed $\omega$ ) and this is clearly

$$
\left(s_{1}^{*}+s_{2}^{*}+\cdots+s_{n}^{*}\right)^{-1 / 2} \sim\left(\int_{0}^{n} e^{b(s)} d s\right)^{-1 / 2}
$$

where $(b(s) \in \mathbb{R} ; s>0)$ denotes standard Brownian motion. The reason why we take $e^{b(s)}$ is that $\alpha \mapsto e^{\alpha}$ is the standard homomorphism between the additive $\mathbb{R}$ and $\mathbb{R}_{+}^{*}$. We then clearly have to take the expectation of the expression (OV.1) demanding in addition that $b(n)$ returns to 0 i.e. we have the estimate

$$
p(t) \sim \mathbb{E}\left[\left(\int_{0}^{t} e^{b(s)} d s\right)^{-1 / 2} ; b(t) \in[-1,1]\right] .
$$

Brownian functionals as this, have become a big industry these days and are being considered by several authors under the glamorous and sexy label of "Financial Mathematics". This is as good a name as any for the flavour of the month, I am sure, but all we need is

$$
p(t) \sim t^{-3 / 2}, \quad t \rightarrow \infty
$$

Instead of considering a random walk on $G$ we can take an analytic point of view. We should consider then $X, X_{*}$ two invariant unit fields along the one parameters subgroups $\mathbb{R}$ and $\mathbb{R}_{+}^{*}$ of $G$ and $\Delta=-X^{2}-X_{*}^{2}$ the corresponding invariant Laplacian. If $\phi_{t}(g)$ denotes the kernel on $G$ of the heat semigroup $e^{-t \Delta}$ generated by $\Delta$ we have

$$
\phi_{t}(g) \sim p(t) \sim t^{-3 / 2}, \quad t \rightarrow \infty .
$$

The task that lies ahead is twofold: 
1) to show that the above geometric construction generalizes to an arbitrary Lie group,

2) to estimate the corresponding Brownian functionals thus obtained.

Both the above tasks are quite formidable at the technical level. In the rest of this chapter, I shall try as much as possible to clarify the general picture.

\section{OV.2. The barrier problem for random walks.}

In this section I shall swich back to standard random walk

$$
S_{n}=X_{1}+\cdots+X_{n} \in \mathbb{R}
$$

where $X_{j}$ are i.i.d. Bernoulli (i.e. $\mathbb{P}\left[X_{j}= \pm 1\right]=1 / 2$ ) variables. The issue in (OV.2) is of course to estimate

$$
p^{*}(n)=\mathbb{E}\left[\left(\sum_{j=1}^{n} e^{S_{j}}\right)^{-A} ; S_{n}=0\right],
$$

So that

$$
p^{*}(n) \leq \mathbb{E}\left(e^{-A M_{n}}, S_{n}=0\right) \sim n^{-3 / 2},
$$

where $M_{n}=\sup _{1 \leq j \leq n} S_{j}$ (recall that $S_{j} \cong-S_{j}$ ). The above asymptotic is obtained because the expectation can be explicitely computed. Indeed there exist standard formulas for the probabilities ( $c f$. [36])

$$
\begin{aligned}
\mathbb{P}\left[M_{n} \leq \lambda ;\right. & \left.S_{n}=0\right] \\
& =\mathbb{P}\left[S_{j}, 1 \leq j \leq n \text { lies below the barrier } \lambda ; S_{n}=0\right] .
\end{aligned}
$$

We can now use the "maximal oscilation"

$$
\operatorname{osc}(t)=\sup _{0<t_{1}, t_{2} \leq t}\left|b\left(t_{1}\right)-b\left(t_{2}\right)\right| ; \quad\left|t_{1}-t_{2}\right| \leq 1,
$$

which is a very "small" variable (and in particular $\|\exp (\operatorname{osc}(t))\|_{L^{1}}=$ $O(t)$ ), and Hölder inequality and we obtain at once that the actual brownian functional (OV.2) (and not just the random walk functional) satisfies

$$
p(n)=O\left(n^{-3 / 2+\varepsilon}\right)
$$


for all $\varepsilon>0$.

We see, in particular, that it is easy to estimate our (OV.2) functional "up to an $\varepsilon$ ". This phenomenon recurs all the time during the whole theory, i.e. the functionals that occur are straight forward to estimate "up to an $\varepsilon$ ". On the other hand to obtain the exact asymptotics becomes rather involved.

\section{OV.3. A generalization.}

The next obvious generalization of the $a x+b$ group is the group $\mathbb{R}^{n} \wedge \mathbb{R}$ where the action of $\mathbb{R}$ on $\mathbb{R}^{n}$ is given by $x \rightarrow e^{\alpha} x(\alpha \in \mathbb{R}$, $x \in \mathbb{R}^{n}$ ). The analysis that we made for $a x+b$ extends "verbatum" to this group. What we have to estimate is the functional

$$
\mathbb{E}\left[\left(\int_{0}^{t} e^{b(s)} d s\right)^{-n / 2} ; b(t) \in[-1,1]\right],
$$

which, as we already saw is also $\sim t^{-3 / 2}$ (or at least $O\left(t^{-3 / 2}\right)$ ). We can push this generalization a step further and consider the group

$$
G=\mathbb{R}^{n}<\mathbb{R}^{a}=V \curlywedge A,
$$

where for simplicity (and since the essential aspects of the problem do not change by this assumption) we shall assume that the action of $A$ on $V$ is semisimple with real roots, i.e. that it is given by $\theta: A \rightarrow G L(V)$ where there exists $L_{1}, \ldots, L_{n} \in A^{*}$ (the dual space) linear functions, that are normally referred to as "roots", such that

$$
\theta(\xi)=\left(\begin{array}{ccc}
e^{L_{1}(\xi)} & & \mathbf{0} \\
& \ddots & \\
\mathbf{0} & & e^{L_{n}(\xi)}
\end{array}\right), \quad \xi \in A=\mathbb{R}^{a} .
$$

By the same analysis it is then very easy to see that the return probability of the corresponding random walk can be estimated by

(OV.4)

$$
\begin{aligned}
p(t)=\mathbb{E}\left[\left(\int_{0}^{t} e^{L_{1}[b(s)]} d s\right)^{-1 / 2}\right. & \\
& \left.\cdots\left(\int_{0}^{t} e^{L_{n}[b(s)]} d s\right)^{-1 / 2} ;|b(s)| \leq 1\right],
\end{aligned}
$$


where now $\left(b(s)=b_{a}(s) \in A=\mathbb{R}^{a} ; s>0\right)$ is the $a$-dimensional standard brownian motion. I do not know whether the functional (OV.4) will help any one make a lot of money with Asian options at the Chicago Exchange. What I do know is that estimates of functionals like this are not easy to get. What we obtain is that (as $t \rightarrow \infty$ )

$$
p(t) \sim t^{-\alpha} \quad \text { or } \quad p(t) \sim e^{-c t^{1 / 3}}
$$

and that it all depends on the geometry of the roots $L_{1}, \ldots, L_{n} \in A^{*}$. There are two types of geometries that we need to consider in this context. The first is the linear geometry i.e. the invariants under $G L(A)$. This allows us to make the following basic classification. We distinguish first the case when the origin $\left(0 \in A^{*}\right)$ lies in the convex combination of the roots $L_{1}, \ldots, L_{n}$. We say then that the roots satisfy the C-condition. And then the NC-case (non-C) which is the opposite situation when all the roots lie strictly on one side of a hyperplane in $A^{*}$. In the C-case we have $p(t) \sim e^{-c t^{1 / 3}}$.

To give a glims of what is happening, let us consider the functional (OV.4) under the C-condition for Brownian motion and for the Bernoulli random walk of Section OV.2 (i.e. $n=2, a=1$; and the roots are: $\left.L_{1}=+1, L_{2}=-1\right)$. We have then

$$
\begin{aligned}
\mathbb{E}\left[\left(\int_{0}^{t} e^{b(s)} d s\right)^{-1 / 2}\right. & \left.\left(\int_{0}^{t} e^{-b(s)} d s\right)^{-1 / 2}\right] \\
& \sim \mathbb{E}\left[\left(\sum_{j=1}^{n} e^{S_{j}}\right)^{-1 / 2}\left(\sum_{j=1}^{n} e^{-S_{j}}\right)^{-1 / 2}\right] \\
& \leq \mathbb{E}\left(\sum_{j=1}^{n} e^{\left|S_{j}\right|}\right)^{-1 / 2} \\
& \leq \mathbb{E}\left[\exp \left(-\frac{1}{2} \sup _{1 \leq j \leq n}\left|S_{j}\right|\right)\right] \\
& =\mathbb{E}\left(e^{-m_{n}}\right),
\end{aligned}
$$

and it is well known and easy to see that with $m(t)=\left(\sup _{0<s<t}|b(s)|\right) / 2$ we have

$$
\mathbb{E}\left(e^{-m(t)}\right) \sim e^{-c t^{1 / 3}} .
$$

In the NC-situation the Euclidean geometry (i.e. the $O(a ; \mathbb{R})=O(A)$ invariants) of $A^{*}$ becomes relevant and we have $p(t) \sim t^{-\alpha}$. The exponent $\alpha$ depends on the "geometry" of the cone

$$
\Omega=\left[x \in \mathbb{R}^{a} ; L_{j}(x)>0, j=1, \ldots, k\right] \subset A \cong \mathbb{R}^{a},
$$


where $L_{1}, \ldots, L_{k}$ are the non zero roots. Observe incidentally that the $\mathrm{NC}$-condition is equivalent to the fact that $\Omega \neq \varnothing$. When all the roots are 0 one should set $\Omega=A$. This geometry, of course, is considered with respect to the Euclidean structure on $A$. At this point we should go back to the definitions of (OV.4) and observe that a Euclidean structure has to be given on $A=\mathbb{R}^{a}$, if brownian motion is to be defined. The question arises what that Euclidean structure is and how is it determined from the group $G$ in (OV.3). The answer to this question is simple: we project the measure $\mu \in \mathbb{P}(G)$ that controls our random walk on $A=\mathbb{R}^{a}$ and take its covariance matrix. This determines the Euclidean structure.

This Euclidean structure is, with hindsight, very natural. It came to me however as a big surprise. First of all this Euclidean structure depends on the random walk on $G$ and not just on $G$. Therefore $t^{-\alpha}$ depends on the measure $\mu$ and, in general, the $\alpha$ varies continously with $\mu$ and can be any large enough real value (e.g. $\left.\alpha=10^{\sqrt{3}} \pi+\sqrt{2}\right)$. This contradicts the intuition that we all had in the subject ( $c f$. [1], [41]) that lead us to believe that $\alpha$ had to be a $1 / 2$-integer. It is worth noting that in $\mathrm{Ph}$. Bougerol's work [41], a natural scalar product does exist in $A$, where $G=N A K$ is the Iwasawa decomposition of some semisimple group $G$. It is given by the Killing form and it gives rise to corresponding $\alpha$ 's that are $1 / 2$-integer. Contrary to what was said, that scalar product and the corresponding $\alpha$ is then independent of the particular measure. This contradiction with what was said above is, however, only apparent. Indeed, for a semisimple group $G$ we have $\mu \in \mathbb{P}(G)$ and not $\mu \in \mathbb{P}(N A)$. The role of the Killing scalar product is important in our theory also. The cone $\Omega$ in (OV.5) should be thought as a generalization of the Weyl chamber of the semisimple theory and the $\alpha \in 1 / 2 \mathbb{Z}$ is related with the symmetries of the Weyl group. This aspect of the theory will not be examined in this paper. The other case when $\alpha \in 1 / 2 \mathbb{Z}$ is, of course, when $G$ is a unimodular NC-group. For these groups we have $\left(L_{1}, L_{2}, \ldots, L_{k}\right)=\varnothing$ since unimodularity amounts to $\sum_{j=1}^{k} L_{j}=0$. It follows that $\Omega=A$ and therefore $\alpha$ is independent of $\mu$. This unimodular theory was developed with different methods in [1].

For the same reasons as in Section OV.2, the functional (OV.4) is intimately connected with the following "conical barrier" problem. Let $x \in \Omega$ (cf. (OV.5)) be fixed, the problem is then to obtain the asymptotics as $t \rightarrow \infty$ of

$$
p_{\Omega}(t)=\mathbb{P}_{x}\left[b_{a}(s) \in \Omega, 0<s<t\right] .
$$


The answer is that there exists some $\lambda=\lambda(\Omega)>0$ such that $p_{\Omega}(t) \sim$ $t^{-\lambda},(t>1)$. The proof of this already takes some doing (cf. [13]).

REMARK. We can bring out the qualitative difference between the Cand NC-geometry at the probabilistic level in the following manner. Consider the region

$$
\Omega^{*}=\left[x \in \mathbb{R}^{a} ; L_{i}(x) \geq-1 ; i=1, \ldots\right],
$$

which is a "polyhedron". This polyhedron is bounded (respectively unbounded) under the C- (respectively NC-) condition and what is relevant in both cases is

$$
p^{*}(t)=\mathbb{P}_{0}\left[b(s) \in \Omega^{*} ; 0<s<t\right] .
$$

The point is that while $p^{*}(t)$ behaves polynomially when $\Omega^{*}$ is unbounded, in the case when $\Omega^{*}$ is bounded we have

$$
p^{*}(t) \sim \mathbb{P}[|b(s)| \leq 1 ; 0<s<t]
$$

which, by the scaling properties of brownian motion $b(s) \in \mathbb{R}^{a}$, is easily seen to have an exponential behaviour (as $t \rightarrow \infty$ ). This is the underlying reason for the difference in behaviour of the Heat kernel under the two geometries.

\section{OV.4. The amenability of the group.}

The analysis that we gave in the previous section extends to all amenable groups. Indeed the model for such a group is a soluble group $P=N \wedge A=N \wedge \mathbb{R}^{a}$ where now $N$ is a general nilpotent group and not just a vector space $V=\mathbb{R}^{n}$. The root analysis of the action of $A$ on $N$ can be carried out as before and the corresponding brownian functionals can be estimated. The details give rise to considerable technical difficulties ( $c f . ~[13],[40]$ ) but not fundamentally new ideas are involved.

New ideas are needed to deal with non amenable (e.g. semisimple) groups. It is these ideas that are developed in this paper. The first hint of how to go about this is supplied by what was already done. The point is that it is not quite exact that we can model a general amenable group by $P=N \wedge A$ as above. The correct model is more like $G=P \wedge K$ where $P$ is the soluble radical and $K$ is a compact semisimple 
Levi factor. These groups can thus be thought as a $P$-principal bundle with a compact base space $G / P=K$. This model generalizes to any connected Lie group $G$ : we can find $P \subset G$ a soluble subgroup such that the homogeneous space $G / P$ is compact. If $G$ is semisimple and $G=N A K$ is the Isawawa decomposition we take $P=N A$. In general we take for $P$ any "Borel" subgroup (here I deviate slightly from the standard terminology). It thus turns out that the correct setting for our theory is to view $G$ as the total space of a $P$-principal bundle with $P$ soluble and $G / P$ compact. There exists then $N \subset P$ a nilpotent normal subgroup such that $P / N \cong \mathbb{R}^{a}=A$, and if we quotient $G$ by the action of $N$ we obtain $X$ an $\mathbb{R}^{a}$-principal bundle. Such a bundle is, topologicaly, trivial, i.e. $X \cong \mathbb{R}^{a} \times K$ (cf. [42]). Observe also that $X$ is a genuine fiber bundle and that it does not admit, in general, a natural group structure.

In this fiber bundle representation of $G$ the Laplacian $\Delta$ on $G$ is identified with a $P$-invariant differential operator on the total space of the bundle. It is in this identification that the factor $e^{-\lambda t}$, where $\lambda$ is the spectral gap of $\Delta$, appears explicitely in the heat diffusion semigroup $e^{-t \Delta}$. It is futile to try to give an intuitive and yet convincing description of how this comes about. But "grosso modo" what happens is that on the fiber bundle $G=P \times K$ (the product is a Borelian trivialization of the bundle) we have to consider both the measures $d^{l} r \otimes d k$ and $d^{r} r \otimes d k$ for left and right Haar measure $d^{l}, d^{r}$. This brings out the modular function $m(x)=d^{r} x / d^{l} x$ and then, somehow, the action of $\Delta$ on $m$ brings out the spectral gap. A similar phenomenom occurs in the construction of the principal series in the representation theory of the semisimple group $G=N A K$. The fact that $P$ is amenable also plays a role here. In the present formalism one should think of $G=N X$ where $X=\mathbb{R}^{a} \times K$ is the generalization of $A=\mathbb{R}^{a}$. As for the brownian motion on $A$ it is replaced by the $\mathbb{R}^{a}$-invariant diffusion on $X$ that is generated on $X$ by $D$, the image of $\Delta$ by $G \rightarrow G / N=X$.

The root analysis of the "action of $X$ on $N$ " can be carried out and the region $\Omega=\left[L_{i}>0, i=1, \ldots\right] \subset X$ can be defined as in (OV.5). One can introduce the analogous brownian functionals and use these functionals to estimate the heat diffusion kernel as before.

The final step that remains is to analyze the second order differential operator $D$ on $X$ and the corresponding "brownian motion" that it generates. 


\section{OV.5. The Laplacian of an $\mathbb{R}^{a}$-principal bundle.}

Let $X=\mathbb{R}^{a} \times K$ or $X=\mathbb{Z}^{a} \times K$ some (trivial) principal bundle with compact base $K$, and let $T$ be some Markovian operator on $X$ that is invariant by the action of $\mathbb{R}^{a}$ or $\mathbb{Z}^{a}$. For instance, we could be looking at the markovian semigroup $T_{t}=e^{-t D}$ on $X=\mathbb{R}^{a} \times K$ as in Section OV.4. Observe that $D$ can be expressed in local coordinates as follows

$$
\begin{aligned}
D=\sum a_{i j}(k) \frac{\partial^{2}}{\partial x_{i} \partial x_{j}} & +\sum b_{i}(k) \frac{\partial}{\partial x_{i}} \\
& +\sum c_{i \alpha}(k) \frac{\partial^{2}}{\partial x_{i} \partial k_{\alpha}}+D\left(k, \frac{\partial}{\partial k}\right),
\end{aligned}
$$

where $D(k, \partial / \partial k)$ only involves the local coordinates $\left(k_{1}, \ldots, k_{j}, \ldots\right)$ of $k \in K$ and $\left(x_{1}, \ldots, x_{a}\right)$ are the (global) coordinates of $\mathbb{R}^{a}$.

I shall denote by

$$
z(n)=\left(z_{R}(n), z_{K}(n)\right) \in \mathbb{R}^{a} \times K=X, \quad n=1,2, \ldots,
$$

the paths of the Markov process generated by $T$.

The simplest non trivial example of the above set up is clearly $X=\mathbb{Z} \times\{0,1\}$ (i.e. when $K=\{0,1\}$ ) is the two point space). $T$ is the determined by $L=(L(i, j) ; i, j=0,1)$ some $2 \times 2$ markovian matrix and by four probability measures $\mu_{i, j} \in \mathbb{P}(\mathbb{Z})(i, j=1,2)$. The Markov chain (OV.7) can then be determined as follows. First the motion of the $K$-coordinate $z_{K}(n)$ is a time homogeneous Markov chain with transition matrix $L$. As for the fiber coordinate $z_{R}(n)$ it moves accordingly to the law

$$
\mathbb{P}\left[z_{R}(n+1)=\zeta^{\prime} / / z(n)=(\zeta, i), z_{K}(n+1)=j\right]=\mu_{i, j}\left(\zeta-\zeta^{\prime}\right),
$$

for $\zeta, \zeta^{\prime} \in \mathbb{Z}, i, j=0,1$. In other words, if we condition the base point at time $n$ to be $i$ and at time $n+1$ to be $j$, then the $n^{\text {th }}$ step on $\mathbb{Z}$ is the same as for a random walk with measure $\mu_{i, j}$. Just as in Section OV.1, therefore, if we condition on the path $z_{K}(0), z_{K}(1), \cdots \in K$, the motion $z_{R}(n)$ becomes a time inhomogeneous random walk on $\mathbb{Z}$. It is clear, of course, that the above description of the process generalizes when $K=\{0,1, \ldots, n-1\}$ has $n$-points or when $K$ is an arbitrary compact space. 
A typical problem that we shall consider for the above process is the following barrier problem: Find the asymptotic behaviour (as $n \rightarrow \infty)$ of

$$
\mathbb{P}_{z(0)=(0,0)}\left[z_{R}(j) \geq-1,1 \leq j \leq n\right],
$$

or more generally when $X=\mathbb{R}^{a} \times K$ and when $\Omega \subset X$ is a connical domain as in (OV.5) and $x \in \Omega$, find the correct asymptotics of

$$
p_{\Omega}(n)=\mathbb{P}_{x}\left[z_{R}(j) \in \Omega ; 1 \leq j \leq n\right] .
$$

The above connical (and "twisted" in the bundle) barrier problem is difficult. Not surprisingly the first step consists in finding $\bar{T}$ the "limit operator" on $\mathbb{R}^{a}$. That operator determines a Markov chain on $\mathbb{R}^{a}$ $\left(\bar{z}(n) \in \mathbb{R}^{a}, n=1,2, \ldots\right)$ that suitably approximates the motion of $z_{R}(n)$ of our process for large times.

The construction of $\bar{T}$ is not trivial. For instance, when $T$ is given by $T_{t}=e^{-t D}$ with $D$ as in (OV.7) then the approximating semigroup is $\bar{T}_{t}=e^{-t \bar{D}}$ with

$$
\bar{D}=\sum \bar{a}_{i j} \frac{\partial^{2}}{\partial x_{i} \partial x_{j}}+\sum \bar{b}_{i} \frac{\partial}{\partial x_{i}}
$$

but it is not, in general, true that the coefficients $\bar{a}_{i j}=\int_{K} a_{i j}(k) d \mu(k)$ are the average of the corresponding coefficients of $D$ with respect to, say, the equilibrium measure $\mu \in \mathbb{P}(K)$ of the $z_{K}(n)$. Finding the above limit operator is a problem from Homogenization Theory ( $c f$. [39]). Once we have determined the limit operator we proceed to show that, when we are in the $\mathrm{NC}$-case (and $\Omega \neq \varnothing$ ), the correct assymptotics in (OV.8) are

$$
p_{\Omega}(t) \sim t^{-\bar{\alpha}}
$$

where $\bar{\alpha}=\bar{\alpha}_{\Omega}$ is the index that corresponds to the cone $\Omega$ and the Euclidean structure determined by $\bar{D}$ as in Section OV.3. If we are in the C-case (i.e. if $\Omega=\varnothing$ ) we obtain, as expected, that $p_{\Omega}(t) \sim e^{-c t^{1 / 3}}$.

The details of the above procedure will not be given in this paper. Only a crude first approximation is given in the Appendix. The full solution will be given in a second instalment of this work.

Solving the above problems is interesting and rewarding because, among other things, they throw new light to classical homogenization theory. 


\section{Algebraic considerations.}

\subsection{Complex soluble algebras and their roots.}

In this section we shall denote by $\mathfrak{q}$ a finite dimensional complex soluble Lie algebra (cf. [5], [6], [7]) and by $\mathfrak{n} \subset \mathfrak{q}$ its nilradical. We shall denote by $\left.\mathfrak{n}^{p}=[\ldots[\mathfrak{n}, \mathfrak{n}] \mathfrak{n}] \ldots \mathfrak{n}\right]$ the $p^{\text {th }}$ commutator, $p=1,2, \ldots$, and by $\mathfrak{k}_{p}=\mathfrak{n}^{p} / \mathfrak{n}^{p+1}$ the corresponding factors. I shall further denote by $W=\mathfrak{k}_{1}, V=\mathfrak{q} / \mathfrak{n}$ and by $\mathfrak{k}=\mathfrak{k}_{1} \oplus \mathfrak{k}_{2} \oplus \cdots, \mathfrak{k}$ is the corresponding graded Lie algebra where, for the canonical multiplication, we have of course $\left[\mathfrak{k}_{j}, \mathfrak{k}_{i}\right] \subset \mathfrak{k}_{i+j}$.

The adjoint action of $\mathfrak{q}$

$$
\operatorname{ad} x: \mathfrak{q} \rightarrow \mathfrak{q}, \quad \text { ad }(x) y=[x, y], \quad x, y \in \mathfrak{q},
$$

induces canonically the following actions

$$
\begin{gathered}
\operatorname{ad} x: \mathfrak{n}^{p} \rightarrow \mathfrak{n}^{p}, \quad p \geq 1, x \in \mathfrak{q}, \\
\operatorname{ad} x: \mathfrak{k}_{p} \rightarrow \mathfrak{k}_{p}, \quad p \geq 1 ; \quad \text { ad } x: \mathfrak{k} \rightarrow \mathfrak{k}, \quad x \in \mathfrak{q} .
\end{gathered}
$$

It is also clear that the action (1.1.2) vanishes if $x \in \mathfrak{n}$. It follows therefore that we also have the following natural actions

$$
\operatorname{ad} v: \mathfrak{k}_{p} \rightarrow \mathfrak{k}_{p}, \quad \text { ad } v: \mathfrak{k} \rightarrow \mathfrak{k}, \quad v \in V=\mathfrak{q} / \mathfrak{n},
$$

and in particular

$$
\text { ad } v: W \rightarrow W, \quad v \in V
$$

$V$ is an abelian Lie algebra, therefore the action (1.1.4) admits the standard root space decomposition

$$
W=W_{1} \oplus \cdots \oplus W_{s},
$$

$$
W_{j}=\left\{x \in W ;\left(\operatorname{ad} v-\lambda_{j}(v)\right)^{N} x=0 ; v \in V\right\}, \quad j=1,2, \ldots, s,
$$

where $\lambda_{j} \in V_{\mathbb{C}}^{*}=\operatorname{Hom}_{\mathbb{C}}[V ; \mathbb{C}]$ are the distinct roots of the action $\left(\lambda_{i} \neq\right.$ $\lambda_{j}, i \neq j, c f$. [5]) and the integer $N$ in (1.1.5) is large enough, say $N=\operatorname{dim} W+10$. 
The actual roots $\lambda_{1}, \ldots \lambda_{s}$ can of course also be defined by the property that for every $j=1, \ldots, s$ there exists $0 \neq \omega \in W$ such that

$$
\left(\lambda_{j}(v)-\operatorname{ad} v\right) \omega=0, \quad v \in V
$$

Analogous root space decompositions exist for the action (1.1.3) so that we have for instance

$$
\mathfrak{k}_{j}=W_{1}^{(j)} \oplus \cdots \oplus W_{s_{j}}^{(j)}, \quad j=1,2, \ldots,
$$

where the root space $W_{r}^{(j)}$ has the root $\lambda_{r}^{(j)}$ and

$$
W_{r}^{(j)}=\sum\left[W_{i_{1}}, W_{i_{2}}, \ldots, W_{i_{j}}\right]
$$

where the summation extends over all indices for which $\lambda_{r}^{(j)}=\lambda_{i_{1}}+$ $\cdots+\lambda_{i_{j}}$ and where in this paper I shall adopt once and for all the following notation

$$
[X, Y, \ldots, Z]=[\ldots[[X, Y] \ldots], Z]
$$

for a higher commutator.

Because of the above situation, as is customary, we shall sometimes say that

$$
\lambda_{1}, \lambda_{2}, \ldots, \lambda_{s}
$$

are the simple roots of the adjoint action and

$$
\lambda_{r}^{(j)}=\lambda_{i_{1}}+\cdots+\lambda_{i_{j}}, \quad j=1, \ldots, r=1, \ldots, s_{j},
$$

are the multiple roots. It is important in what follows to examine more closely the above roots and to give what amounts to alternative definitions of the above notions.

Since $\mathfrak{q}$ is soluble, the action (1.1.1) (for $p=1$ ) can be simultaneously triangulated ( $c f .[5],[6])$. In other words we can choose a basis of $\mathfrak{n}$ with respect to which the action (1.1.1) takes the form

$$
\operatorname{ad} x=\left(\begin{array}{ccc}
\nu_{1}(x) & & * \\
& \ddots & \\
\mathbf{0} & & \nu_{t}(x)
\end{array}\right) \text {. }
$$


In (1.1.8) $\nu_{j} \in \operatorname{Hom}_{\mathbb{C}}[\mathfrak{q} ; \mathbb{C}]=\mathfrak{q}_{\mathbb{C}}^{*}$ are complex linear functionals on $\mathfrak{q}$ that vanish identically on $\mathfrak{n}$ and can thus be identified with elements of $V_{\mathbb{C}}^{*}$.

By the standard Jordan-Hölder theorem on composition series [8] we see then that up to a different order the $\nu_{1}, \ldots, \nu_{t} \in V_{\mathbb{C}}^{*}$ are exactly the roots $\lambda_{r}^{(j)}$ in (1.1.7).

The third definition of the roots is less elementary. Let $\mathfrak{h} \subset \mathfrak{q}$ be some Cartan subalgebra of $\mathfrak{q}(c f . \quad[5],[7])$ or more generally just some nilpotent subalgebra of $\mathfrak{q}$ that has the additional property (Cartan subalgebras have that property $(c f .[7]))$,

$$
\mathfrak{n}+\mathfrak{h}=\mathfrak{q}
$$

We can then consider the root space decomposition

$$
\mathfrak{n}=\mathfrak{n}_{1} \oplus \mathfrak{n}_{2} \oplus \cdots \oplus \mathfrak{n}_{\ell}
$$

of the ad-action of $\mathfrak{h}$ on $\mathfrak{n}$ where as before

$$
\mathfrak{n}_{j}=\left\{y \in \mathfrak{n}:\left(\operatorname{ad} x-\mu_{j}(x)\right)^{N} y=0, \text { for all } x \in \mathfrak{h}\right\}
$$

(cf. [5]) with $\mu_{j} \in \mathfrak{h}_{\mathbb{C}}^{*}=\operatorname{Hom}_{\mathbb{C}}[\mathfrak{h}, \mathbb{C}]$ as before. The important thing here is that

$$
\left[\mathfrak{n}_{i}, \mathfrak{n}_{j}\right] \subset \mathfrak{n}_{k},
$$

where $\mu_{i}+\mu_{j}=\mu_{k}$ (cf. [5]) and that, since the $\mu_{j}$ 's vanish identically on $\mathfrak{h} \cap \mathfrak{n}$, we can identify these $\mu$ 's to elements of $(\mathfrak{h} / \mathfrak{h} \cap \mathfrak{n})_{\mathbb{C}}^{*}=V_{\mathbb{C}}^{*}$ because of (1.1.9).

Therefore $\mu_{1}, \ldots, \mu_{\ell}$ can be identified with elements of $V_{\mathbb{C}}^{*}$, and by the same composition series arguments (applied to the action of $\mathfrak{h}$ on n) we can identify the $\mu_{1}, \ldots, \mu_{\ell}$ (up to a new order) with the roots (1.1.7).

\subsection{Real soluble algebras and their roots.}

In this section I shall denote by $\mathfrak{q}$ a finite dimensional real soluble Lie algebra and by $\mathfrak{n} \subset \mathfrak{q}$ its nilradical. I shall fix $\mathfrak{h}$ some Cartan subalgebra (or more generally some nilpotent subalgebra that satisfies (1.1.9)) and I shall denote by $\mathfrak{q}_{c}=\mathfrak{q} \otimes \mathbb{C}, \mathfrak{n}_{c}=\mathfrak{n} \otimes \mathbb{C}$ and $\mathfrak{h}_{c}=\mathfrak{h} \otimes \mathbb{C}$ the corresponding complexified algebras. It is then well known that $\mathfrak{n}_{c}$ 
is the nilradical of $\mathfrak{q}_{c}$ (it is also true, but irrelevant for us here, that $\mathfrak{h}_{c}$ is a Cartan subalgebra of $\mathfrak{q}_{c}$ if $\mathfrak{h}$ is a Cartan subalgebra of $\mathfrak{q}$ ). Let us also follow the same notations as in Section 1.1 and denote by

$$
V=\mathfrak{q} / \mathfrak{n}, \quad W=\mathfrak{n} /[\mathfrak{n}, \mathfrak{n}]
$$

which are real vector spaces. We then have canonical identifications

$$
V_{c}=V \otimes \mathbb{C}=\mathfrak{q}_{c} / \mathfrak{n}_{c}, \quad W \otimes \mathbb{C}=\mathfrak{n}_{c} /\left[\mathfrak{n}_{c}, \mathfrak{n}_{c}\right]
$$

Relative to the complex algebra $\mathfrak{q}_{c}$ the roots (1.1.6) can then be identified with (I denote by $t$ and not by $s$ the number of these roots here)

$$
\lambda_{1}, \ldots, \lambda_{t} \in \operatorname{Hom}_{\mathbb{R}}[V ; \mathbb{C}]
$$

that are defined by the property that there exists $0 \neq \omega \in W \otimes \mathbb{C}$ such that

$$
\left(\lambda_{j}(x)-\operatorname{ad} x\right) \omega=0, \quad \text { for all } x \in V .
$$

At this stage it is important to introduce a notation that we shall adopt throughout. The real algebra $\mathfrak{q}$ induces a "real structure" (i.e. a "complex conjugation", $c f .[9],[10])$ in the complex algebra $\mathfrak{q}_{c}=\mathfrak{q} \otimes \mathbb{C}$. I shall consider the complex subalgebras (or even complex subspaces) of $\mathfrak{q}_{c}$ that respect to the above real structure (i.e. are stable by the above complex conjugation). I shall reserve the suffix $c$ to indicate by $\mathfrak{a}_{c} \subset \mathfrak{q}_{c}$ these subalgebras. This means that $\mathfrak{a}_{c}=\mathfrak{a} \otimes \mathbb{C}$ for some subalgebra $\mathfrak{a} \subset \mathfrak{q}$. Such subspaces will be called real.

The considerations of Section 1.1 apply to $\mathfrak{q}_{c}$ and the subalgebra $\mathfrak{h}_{c}$. The important thing is to "build" in the definition of the roots the above real structure. The key definition needed to do that is that of the real simple roots or simply the real roots when confusion does not arise.

These are $L_{1}, \ldots, L_{s} \in V^{*}$ (= the real dual of $V$ ) which are the distinct non zero real parts of the roots $\lambda_{1}, \ldots, \lambda_{t}$ of (1.2.1) i.e.

$$
L(v)=\operatorname{Re} \lambda(v), \quad v \in V .
$$

We can of course consider the graded algebra

$$
\mathfrak{k}_{c}=\bigoplus_{p}\left(\mathfrak{n}_{c}\right)^{p} /\left(\mathfrak{n}_{c}\right)^{p+1}
$$


and the corresponding action of $V_{c}$ on $\mathfrak{k}_{c}$. The "multiple" real roots can thus be defined in the obvious way and these are finite linear combinations with positive integer coefficients of the $L_{j}$ 's

$$
L_{r}^{(j)}=L_{i_{1}}+L_{i_{2}}+\cdots+L_{i_{\alpha}} \neq 0
$$

(with the $i$ 's not necessarily all distinct).

Let us denote by

$$
\mathcal{L}=\left\{\sum_{j=1}^{s} \alpha_{j} L_{j}: \alpha_{j} \geq 0, \sum_{j=1}^{s} \alpha_{j}=1\right\} \subset V^{*}
$$

the convex hull in $V^{*}$ of the real roots with the understanding that $\mathcal{L}=\varnothing$ if

$$
\left\{L_{1}, L_{2}, \ldots, L_{s}\right\}=\varnothing
$$

Definition. We shall say that the algebra $\mathfrak{q}$ is a $\mathrm{C}$-algebra if $0 \in \mathcal{L}$, otherwise (if $0 \notin \mathcal{L}$ ) we shall say that $\mathfrak{q}$ is an $\mathrm{NC}$-algebra.

Algebras for which (1.2.3) holds are called R-algebras ( $c f$. [11]). $\mathrm{R}$-algebras are in particular NC-algebras. It should finally be observed that in the above definition nothing changes (i.e. we obtain the same classification of C-, NC-algebras) if we replace $\mathcal{L}$ by $\overline{\mathcal{L}}$ the corresponding convex hull in $V^{*}$ of the "multiple" real roots (1.2.2) which are just the non zero real parts $\operatorname{Re} \lambda_{r}^{(j)}$ of the (multiple) roots $\lambda_{r}^{(j)}$ in (1.1.7).

Let us recall that quite generally we say that the Lie algebra $\mathfrak{g}$ is unimodular if

$$
\operatorname{trace}(\operatorname{ad} x)=0, \quad x \in \mathfrak{g} .
$$

It follows at once that if $\mathfrak{q}$ as above is unimodular and satisfies the NC-condition then (1.2.3) holds and $\mathfrak{q}$ is an R-algebra.

\subsection{The structure of soluble NC-algebras.}

In this section $\mathfrak{n}, \mathfrak{h} \subset \mathfrak{q}$ will be as in Section 1.2. All the notations of Section 1.2 will be preserved and we shall consider the root space decomposition

$$
\mathfrak{n}_{c}=\mathfrak{n}_{0}^{(c)} \oplus \cdots \oplus \mathfrak{n}_{\ell}^{(c)}
$$


of $\mathfrak{n}_{c}$ under the ad-action of $\mathfrak{h}_{c}$. In this decomposition (1.3.1) if $\mu=0$ is a root as in (1.1.10) we denote by $\mathfrak{n}_{0}^{(c)}$ the root space corresponding to that root. When $\mu=0$ is a root then the space $\mathfrak{n}_{0}^{(c)}$ is a real space and we have

$$
\mathfrak{n}_{0}^{(c)}=\left(\tilde{\mathfrak{n}}_{0}\right)_{c},
$$

where $\tilde{\mathfrak{n}}_{0} \subset \mathfrak{n}$. Observe also that $\mathfrak{h}_{c} \cap \mathfrak{n}_{c} \subset \mathfrak{n}_{0}^{(c)}$ and therefore that

$$
\mathfrak{n} \cap \mathfrak{h} \subset \tilde{\mathfrak{n}}_{0}
$$

when $\mu=0$ is not a root we shall abusively set $\tilde{\mathfrak{n}}_{0}=0$.

The other root spaces $\mathfrak{n}_{j}^{(c)}$ are of course not necessarily real. We shall therefore partition all the roots $\mu_{1}, \ldots, \mu_{\ell}$ into disjoint subsets by the equivalence relation

$$
\mu_{i} \sim \mu_{j} \quad \text { if and only if } \quad \operatorname{Re} \mu_{i}=\operatorname{Re} \mu_{j}
$$

and block together the corresponding subspaces. We obtain thus a direct decomposition

$$
\mathfrak{n}=\mathfrak{n}_{0} \oplus \mathfrak{n}_{1} \oplus \cdots \oplus \mathfrak{n}_{k}
$$

where $\left(\mathfrak{n}_{i}\right)_{c}=\mathfrak{n}_{i_{1}}^{(c)} \oplus \cdots \oplus \mathfrak{n}_{i_{\alpha}}^{(c)}$ with $\mu_{i_{1}}, \ldots, \mu_{i_{\alpha}}$ the roots in the equivalence class $\operatorname{Re} \mu_{i_{1}}=\operatorname{Re} \mu_{i_{2}}=\cdots=\operatorname{Re} \mu_{i_{\alpha}}=L_{i}$.

In the notations of (1.3.4) we shall (abusively) assume that $\mathfrak{n}_{0}$ may be $=\{0\}$ and will always correspond to the equivalence class $\operatorname{Re} \mu=0$. We have of course

$$
\tilde{\mathfrak{n}}_{0} \subset \mathfrak{n}_{0}, \quad\left[\mathfrak{n}_{i}, \mathfrak{h}\right] \subset \mathfrak{n}_{i}, \quad i=0,1, \ldots, k
$$

Finally for any two $i, j=0,1, \ldots, k$ we have

$$
\left[\mathfrak{n}_{i}, \mathfrak{n}_{j}\right] \subset \mathfrak{n}_{p}
$$

where in the equivalence class of the roots of $\mathfrak{n}_{p}$ the real part is the sum of the two corresponding real parts $(c f$. [5]). The following important proposition immediately follows from (1.3.2), (1.3.5), (1.3.6) and (1.1.9).

Proposition. If we assume that $\mathfrak{q}$ is an NC-algebra then $\mathfrak{n}_{R}=\mathfrak{n}_{1} \oplus$ $\cdots \oplus \mathfrak{n}_{k}$ is an ideal in $\mathfrak{q}$ and $\mathfrak{q}_{R}=\mathfrak{n}_{0}+\mathfrak{h}$ is a subalgebra such that 
$\mathfrak{n}_{R} \cap \mathfrak{q}_{R}=\{0\}$ and $\mathfrak{q}=\mathfrak{n}_{R}+\mathfrak{q}_{R}$. In other words we have a semidirect product decomposition

$$
\mathfrak{q}=\mathfrak{n}_{R} \curlywedge \mathfrak{q}_{R}
$$

Furthermore, we have

$$
\left[\mathfrak{n}_{j}, \mathfrak{q}_{R}\right] \subset \mathfrak{n}_{j}, \quad j=1, \ldots, k
$$

It is clear that $\mathfrak{q}_{R}$ is a soluble R-algebra (i.e. it satisfies (1.2.3)). Observe that quite generally if two ideals $\mathfrak{j}_{1}, \mathfrak{j}_{2} \subset \mathfrak{q}$ have the property

$$
\mathfrak{q} / \mathfrak{j}_{i} \quad \text { is an R-algebra }
$$

then the ideal $\mathfrak{j}_{1} \cap \mathfrak{j}_{2}$ has the same property. Indeed $\mathfrak{q} / \mathfrak{j}_{1} \cap \mathfrak{j}_{2}$ can be identified to a subalgebra of $\mathfrak{q} / \mathfrak{j}_{1} \times \mathfrak{q} / \mathfrak{j}_{2}$ which is an R-algebra. It follows in particular that the ideal $\mathfrak{n}_{R} \subset \mathfrak{q}$ can be given an intrinsic characterization and is the smallest ideal $\mathfrak{j}$ that has the property (1.3.9). It is in particular independent of the choice of $\mathfrak{h}$.

We shall finally need to examine more closely the action (1.3.7). The algebra $\mathfrak{q}_{R}$ is soluble. For every fixed $j$ we can therefore chose a basis over $\mathbb{C}$ on $\left(\mathfrak{n}_{j}\right)_{c}=\mathfrak{n}_{j} \otimes_{\mathbb{R}} \mathbb{C}$ in such a way that with respect to that basis we have

$$
\operatorname{ad} x=\left(\begin{array}{ccc}
\rho_{1}(x) & & * \\
& \ddots & \\
\mathbf{0} & & \rho_{t_{j}}(x)
\end{array}\right), \quad x \in \mathfrak{q}_{R},
$$

where the $\rho_{k}$ 's vanish identically on $\mathfrak{n} \cap \mathfrak{h}$ since ad $x$ is a nilpotent tranformation for $x \in \mathfrak{n} \cap \mathfrak{h}$. The $\rho_{k}$ 's can thus be identified with elements of $(\mathfrak{h} / \mathfrak{h} \cap \mathfrak{n})_{\mathbb{C}}^{*}=\operatorname{Hom}_{\mathbb{R}}[\mathfrak{h} / \mathfrak{h} \cap \mathfrak{n} ; \mathbb{C}]=(\mathfrak{q} / \mathfrak{n})_{\mathbb{C}}^{*}$ and can thus be identified with the elements of the equivalence class of the roots $\mu$ of (1.3.3) that have a fixed non zero real part.

\subsection{A general Lie algebra and the Levi decomposition.}

In this section I shall consider a general finite dimensional real Lie algebra $\mathfrak{g}$ and I shall denote by $\mathfrak{n} \subset \mathfrak{q} \subset \mathfrak{g}$ its radical and its nilradical 
(cf. [5], [6]). We can then find $\mathfrak{s} \subset \mathfrak{g}$ a semisimple subalgebra (with the convention that $\mathfrak{s}$ could be $=\{0\}$ ) such that

$$
\mathfrak{g}=\mathfrak{q} \wedge \mathfrak{s}
$$

This is of course the standard Levi decomposition and $\mathfrak{s}$ is called a Levi subalgebra of $\mathfrak{g}(c f .[5],[6],[7])$. The following lemma was first proved and successfully exploited by G. Alexopoulos [12]. The proof I give below is different

Lemma (Alexopoulos [12]). We can find $\mathfrak{h}_{0} \subset \mathfrak{q}$ a nilpotent subalgebra such that

$$
\mathfrak{q}=\mathfrak{n}+\mathfrak{h}_{0}, \quad\left[\mathfrak{s}, \mathfrak{h}_{0}\right]=\{0\}
$$

Proof. By H. Weyl's theorem (cf. [5], [6]) on the semisimplicity of a representation of any semisimple algebra, we can find $\mathfrak{l} \subset \mathfrak{q}$ a subspace such that $\mathfrak{q}=\mathfrak{n} \oplus \mathfrak{l}$ and such that $[\mathfrak{s}, \mathfrak{l}] \subset \mathfrak{l}$. But since $[\mathfrak{g}, \mathfrak{q}] \subset \mathfrak{n}(c f .[6])$ we have $[\mathfrak{s}, \mathfrak{l}]=0$. This means that

$$
\mathfrak{l} \subset \mathfrak{q}_{0}=\{x \in \mathfrak{q}:[\mathfrak{s}, x]=0\},
$$

where $\mathfrak{q}_{0}$ is a subalgebra of $\mathfrak{q}$. If follows in particular that $\mathfrak{t}_{0}=\mathfrak{q}_{0} / \mathfrak{q}_{0} \cap$ $\mathfrak{n}=\mathfrak{q} / \mathfrak{q} \cap \mathfrak{n}$. If we set $\mathfrak{h}_{0} \subset \mathfrak{q}_{0}$ to be some Cartan subalgebra of $\mathfrak{q}_{0}$ we see therefore that all the conditions of the lemma are verified because the canonical image of $\mathfrak{h}_{0}$ in $\mathfrak{t}_{0}$ is $\mathfrak{t}_{0}(c f .[7])$.

The subalgebra $\mathfrak{h}_{0}$ is not in general a Cartan subalgebra of $\mathfrak{q}$ but what the lemma says is that it satisfies the condition (1.1.9). It follows therefore that we can make all the constructions of Section 1.3 starting from the algebra $\mathfrak{h}_{0}$. Using this we shall extend our previous definition to general algebras.

Definition. Let $\mathfrak{q} \subset \mathfrak{g}$ be as above. We shall say that $\mathfrak{g}$ is a C(respectively $\mathrm{NC}-$ ) algebra if $\mathfrak{q}$ is.

It follows that if with the above definition $\mathfrak{g}$ is an NC-algebra then we can define the ideal $\mathfrak{n}_{R} \subset \mathfrak{q}$ and decompose $\mathfrak{q}=\mathfrak{n}_{R} \wedge \mathfrak{q}_{R}$ where $\mathfrak{q}_{R}$ is defined as in proposition of Section 1.3 and depends on the choice 
of $\mathfrak{h}_{0}$ (as we already pointed out $\mathfrak{n}_{R}$ does not depend on that choice). The fact that $\left[\mathfrak{h}_{0}, \mathfrak{s}\right]=\{0\}$ implies that in the decomposition (1.3.1) all the subspaces $\mathfrak{n}_{j}^{(c)}$ are stable by the ad-action of $\mathfrak{s}$. Therefore it follows (with the notations of Section 1.3) that $\tilde{\mathfrak{n}}_{0}$ and all the subspaces $\mathfrak{n}_{j}$ in (1.3.4) are stable by the ad-action of $\mathfrak{s}$

$$
\left[\tilde{\mathfrak{n}}_{0}, \mathfrak{s}\right] \subset \tilde{\mathfrak{n}}_{0}, \quad\left[\mathfrak{n}_{j}, \mathfrak{s}\right] \subset \mathfrak{n}_{j}, \quad j=0,1, \ldots, s .
$$

We obtain thus the semidirect product decomposition

$$
\mathfrak{g}=\mathfrak{n}_{R} \curlywedge\left(\mathfrak{q}_{R} \curlywedge \mathfrak{s}\right) .
$$

Observe finally that when $\mathfrak{s}$ is of compact type and therefore $\mathfrak{q}_{R}<\mathfrak{s}$ is an R-algebra then $\mathfrak{n}_{R}$ can be characterized as before as the smallest ideal $\mathfrak{j} \subset \mathfrak{g}$ for which $\mathfrak{g} / \mathfrak{j}$ is an $R$-algebra. A final observation is in order. We have

$$
\mathfrak{q}_{R}<\mathfrak{s}=\left(\mathfrak{n}_{0}+\mathfrak{h}_{0}\right)<\mathfrak{s}
$$

and $\mathfrak{n}_{0} \subset \mathfrak{q}_{R} \curlywedge \mathfrak{s}$ is an ideal by (1.4.3) and we can consider the projection

$$
\pi: \mathfrak{q}_{R} \curlywedge \mathfrak{s} \rightarrow\left(\mathfrak{q}_{R} \curlywedge \mathfrak{s}\right) / \mathfrak{n}_{0}=\left(\mathfrak{h}_{0} / \mathfrak{h}_{0} \cap \mathfrak{n}_{0}\right) \oplus \mathfrak{s}=\pi\left(\mathfrak{q}_{R} \curlywedge \mathfrak{s}\right)
$$

i.e. $\mathfrak{s}$ and $\mathfrak{h}_{0} / \mathfrak{h}_{0} \cap \mathfrak{n}_{0}$ commute in $\pi(\mathfrak{q} R / \mathfrak{s})$. This is because $[\mathfrak{g}, \mathfrak{q}] \subset \mathfrak{n}$ (cf. [6]) and (1.4.2), (1.4.4).

\subsection{A lemma from linear algebra.}

In this section I shall consider

$$
M_{j}=D_{j}+T_{j} \in M_{n \times n}(\mathbb{C}), \quad j=1, \ldots, s .
$$

A finite number of complex invertible matrices where $D_{j}=\operatorname{Diag}\left(d_{1}^{(j)}\right.$, $\left.\ldots, d_{n}^{(j)}\right)$ is assumed diagonal with diagonal entrees $d_{i}^{(j)} \neq 0$ and $T_{j}=$ $\left(t_{\alpha, \beta}^{(j)}\right)_{\alpha, \beta=1}^{n}$ is assumed upper triangular i.e. we assume that $t_{\alpha, \beta}^{(j)}=0$ $(j=1, \ldots, s, \alpha \geq \beta)$. I shall set

$$
\max _{1 \leq j \leq s}\left\{\left\|M_{j}\right\|,\left\|M_{j}^{-1}\right\|\right\}=e^{u}, \quad u \geq 0
$$


where $\|\cdot\|$ indicates the operator norm of the matrix (with respect to the canonical hermitian scalar product $\sum z_{i} \bar{u}_{i}$ on $\mathbb{C}^{n}$ ). I shall also set

$$
\delta_{j}=\max _{1 \leq i \leq n}\left|d_{i}^{(j)}\right|, \quad \delta_{1} \delta_{2} \cdots \delta_{s}=e^{\rho}, \quad \rho \in \mathbb{R} .
$$

(For our applications $(c f .(1.6 .5),(1.6 .7))$ we are in fact going to have $\left.\delta_{j}=\left|d_{i}^{(j)}\right|, i=1,2, \ldots\right)$

What will be proved in this section is that there exists $C$ some numerical constant such that

$$
\left\|M_{1} \cdots M_{s}\right\| \leq C^{n} s^{n} \exp (C n u+\rho)
$$

First of all we shall reduce the proof of (1.5.1) to the special case $\delta_{j}=1$, $j=1, \ldots, s$ where $(1.5 .1)$ reduces to

$$
\left\|M_{1} \cdots M_{s}\right\| \leq C^{n} s^{n} e^{C n u} .
$$

Indeed we clearly have $\delta_{j}^{-n} \leq\left|d_{1}^{(j)} \cdots d_{n}^{(j)}\right|^{-1}=\operatorname{det}\left(M_{j}^{-1}\right) \leq e^{n u}$. Therefore $\delta_{j}^{-1} \leq e^{u}$ and since trivially $\delta_{j} \leq e^{u}$ the new matrix $\tilde{M}_{j}=$ $\delta_{j}^{-1} M_{j}$ satisfy $\left\|\tilde{M}_{j}\right\|,\left\|\tilde{M}_{j}^{-1}\right\| \leq e^{2 u}$. The $\tilde{\delta}_{j}$ that correspond to these new matrices clearly satisfy $\tilde{\delta}_{j}=1$ and we are in the special case. The estimate (1.5.2) for these new matrices immediately implies the general result (1.5.1). It remains to give a proof of (1.5.2). Let us develop the product

$$
\prod_{j=1}^{s}\left(D_{j}+T_{j}\right)=\sum_{\substack{\varepsilon_{k}= \pm 1 \\ k=1, \ldots, s}} A_{1}^{\left(\varepsilon_{1}\right)} \cdots A_{s}^{\left(\varepsilon_{s}\right)}
$$

where $A_{j}^{(+1)}=D_{j}, A_{j}^{(-1)}=T_{j}, j=1,2, \ldots, s$.

It is clear furthermore that every term of the form $A_{1}^{\left(\varepsilon_{1}\right)} \cdots A_{s}^{\left(\varepsilon_{s}\right)}$ is 0 if among the $\varepsilon_{j}$ 's we can find at least $n+1(-1)$ 's. It follows that in the summation of the right hand side of (1.5.3) there are at most $s^{n}$ non zero terms and since we clearly have $\left\|A_{j}^{(\varepsilon)}\right\| \leq 2 e^{C u}$ our estimate (1.5.2) follows.

In words what the estimate (1.5.1) says is the following: the norm of $\left\|M_{1} \cdots M_{s}\right\|$ which has the obvious exponential bound $e^{s u}$ can in fact be estimated by $\delta_{1} \cdots \delta_{s}$ (this in general does grow exponentially in $s$ but it does so in a special way!) multiplied by a polynomial in $s$. 


\subsection{The geometric interpretation of the lemma for soluble Lie groups.}

In this section we shall consider $Q$ a real soluble connected Lie group (that is not assumed to be simply connected) and let

$$
\pi: Q \rightarrow G L_{n}(\mathbb{R})
$$

a $n$-dimensional real representation of $Q$. I shall denote by $\mathfrak{q}$ the Lie algebra of $Q$ and by

$$
d \pi: \mathfrak{q} \rightarrow \mathfrak{g} \ell_{n}(\mathbb{R})=\operatorname{End}_{\mathbb{R}}\left(\mathbb{R}^{n}\right)
$$

the corresponding representation. The above representations can then be complexified and a basis over $\mathbb{C}$ can be chosen on $\mathbb{C}^{n}$ in such a way that $d \pi(x)(x \in \mathfrak{q})$ is upper triangular

$$
d \pi(x)=\left(\begin{array}{ccc}
\xi_{1}(x) & & * \\
& \ddots & \\
\mathbf{0} & & \xi_{n}(x)
\end{array}\right)=m \in M_{n \times n}(\mathbb{C}), x \in \mathfrak{q}
$$

The $\xi_{i}$ 's are of course elements of $\operatorname{Hom}_{\mathbb{R}}(\mathfrak{q} ; \mathbb{C})$. If $g=\operatorname{Exp}(x) \in Q$ where

$$
\operatorname{Exp}: \mathfrak{q} \rightarrow Q
$$

is the standard exponential mapping from the Lie algebra $\mathfrak{q}$ in the group $Q$ (this exponential mapping is not in general "onto") we have

$$
\begin{aligned}
\pi(g) & =\exp m=M \\
& =\left(\begin{array}{ccc}
\Xi_{1}(g) & & * \\
& \ddots & \\
\mathbf{0} & & \Xi_{n}(g)
\end{array}\right) \in G L_{n}(\mathbb{C}), \quad g \in \operatorname{Exp}(\mathfrak{q}),
\end{aligned}
$$

where $\Xi_{j}(g)=e^{\xi_{j}(x)}$ and

$$
\left|\Xi_{j}(g)\right|=e^{L_{j}(g)}=e^{\operatorname{Re} \xi_{j}(x)}, \quad g=\operatorname{Exp}(x), x \in \mathfrak{q} .
$$

It follows (since $\operatorname{Exp}(\mathfrak{q})$ generates $Q$ ) that $\pi(g)$ can be simultaneously triangulated for all $g \in Q$ and that $g \rightarrow \Xi_{j}(g)$ is a global homomorphism 
$Q \rightarrow \mathbb{C}^{*}=\mathbb{C} \backslash\{0\}$ (with the multiplicative structure). This also defines a group homomorphism

$$
Q \rightarrow \mathbb{R}, \quad g \mapsto L_{j}(g)
$$

What the estimate (1.5.1) says in this context is that if $g_{1}, \ldots, g_{s} \in Q$ are such that $\left|g_{j}\right| \leq u, j=1, \ldots, s$ (cf. [1] and Section 3.1 for the definition of $\left.|g|=\left|g^{-1}\right|\right)$ and if $L_{1}=L_{2}=\cdots=L$ and

$$
L\left(g_{1} \cdots g_{s}\right) \leq \rho, \quad \rho \in \mathbb{R}
$$

then

$$
\left\|\pi\left(g_{1} \cdots g_{s}\right)\right\| \leq C^{n} s^{n} \exp (C n u+\rho) .
$$

Let us illustrate the above considerations further in terms of NC-algebras. Let us assume that $\mathfrak{q}$ is a real $\mathrm{NC}$-algebra and let

$$
\mathfrak{q}=\mathfrak{n}_{R} \curlywedge \mathfrak{q}_{R}
$$

be the decomposition (1.3.7) that corresponds to some choice of $\mathfrak{h} \subset \mathfrak{q}$. I shall denote by $Q$ the simply connected real Lie group that corresponds to the algebra $q$. The analytic subgroup $N_{R} \subset Q$ that corresponds to the ideal $\mathfrak{n}_{R}$ is clearly closed and simply connected ( $c f$. [6]). We can also construct "ad hoc" $Q_{R}$ the simply connected Lie group whose algebra is $\mathfrak{q}_{R}$. The group $Q_{R}$ acts canonically (as a group of automorphisms) on $N_{R}$. Indeed for $\Omega \subset Q_{R}$ a small enough neighbourhood of the identity we define that action by the obvious inner automorphism. The simple connectedness of $Q_{R}$ does the rest. We can define thus the semidirect product $N_{R} \wedge Q_{R}$ and the simple connectedness of $G$ implies that we can identify

$$
Q=N_{R} \curlywedge Q_{R}
$$

and that $Q_{R}$ can be identified to the analytic subgroup of $Q$ that corresponds to the subalgebra $\mathfrak{q}_{R} . Q_{R}$ is thus a closed subgroup.

We can apply our previous considerations to the representations $(1.6 .1),(1.6 .2)$

$$
\left\{\begin{array}{l}
\pi=\operatorname{Ad}: Q_{R} \rightarrow G L\left(\mathfrak{n}_{R}\right), \\
d \pi=\operatorname{ad}: \mathfrak{q}_{R} \rightarrow \mathfrak{g} \ell\left(\mathfrak{n}_{R}\right),
\end{array}\right.
$$

(recall that $\operatorname{Ad} g=\left.d I_{g}\right|_{e}, I_{g} x=g^{-1} x g, g, x \in G, c f .[6]$ ). 
Let us now consider $N_{0} \subset Q_{R}$ the closed subgroup that corresponds to the ideal $\mathfrak{n}_{0} \subset \mathfrak{q}_{R}=\mathfrak{n}_{0}+\mathfrak{h}$. For the above example (1.6.6) the elements $d \pi(x)\left(x \in \mathfrak{n}_{0}\right)$ are nilpotent transformations and therefore all the $\xi_{j}$ 's of (1.6.3) vanish identically on $\mathfrak{n}_{0}$. It follows that the $L_{j}$ 's define in (1.6.4) factor through $\kappa: Q_{R} \rightarrow Q_{R} / N_{0}$ and can be considered as group homomorphisms

$$
L_{j}: Q / N \cong Q_{R} / N_{0} \rightarrow \mathbb{R}
$$

where now $N \subset Q$ is the nilradical of $Q$. The results in Section 1.5 give then here the following estimate:

Let $g_{1}, \ldots, g_{s} \in Q_{R} \subset Q$ and let assume that $\left|g_{j}\right| \leq u(j=1, \ldots, s)$ (observe that ||$_{Q}$ and ||$_{Q_{R}}$ are equivalent $c f$. Chapter 3 ) and that $L_{k}\left[\kappa\left(g_{1} \cdots g_{s}\right)\right] \leq \rho, k=1, \ldots, n$. Then

$$
\left\|\left.\operatorname{Ad}\left(g_{1} \cdots g_{s}\right)\right|_{\mathfrak{n}_{R}}\right\| \leq C^{n} s^{n} \exp (C n u+\rho) .
$$

The condition $L_{1}=L_{2}=\cdots$, that was needed for the validity of (1.6.5), is here guaranteed by (1.3.8). Indeed it is on each $\mathfrak{n}_{j},(j=1, \ldots, k)$ separately, that we apply our Lemma.

Let now $q_{1}, \ldots, q_{s} \in Q_{R}$ be as before and let us assume that: $\left|q_{j}\right| \leq u, j=1, \ldots, s ; L_{k}\left(\kappa\left(q_{1} \cdots q_{i}\right)\right) \leq \rho, i=1, \ldots, s-1, k=1, \ldots, n$.

Let further

$$
B(r)=\left\{n \in N:|n|_{N} \leq r\right\}
$$

denote the $r$-ball in $N$. We then clearly have

$$
\begin{aligned}
\mathcal{B} & =B(r) q_{1} B(r) q_{2} \cdots B(r) q_{s} \\
& =\left(B(r) B(r)^{q_{1}} B(r)^{q_{1} q_{2}} \cdots B(r)^{q_{1} \cdots q_{s-1}}\right) q_{1} \cdots q_{s},
\end{aligned}
$$

where as usual for any group $G$ we set $g^{h}=h g h^{-1}(g, h \in G)$. It follows therefore from (1.6.7) and (1.6.8) (cf. [13]) that

$$
\mathcal{B} \subseteq B(R) q_{1} \cdots q_{s}
$$

where $R=r C^{n} s^{n+1} \exp (C n u+\rho)$. The estimate (1.6.9) implies in particular that

$$
|n|_{N} \leq C \exp \left(C|n|_{G}\right), \quad n \in N \subset Q
$$

A fact that as we shall point out in Section 3.1 holds in general. 
In the spirit of Section 1.4 the above considerations extend to a general Lie group $G$ that it is not necessarily soluble. No use of this will be made in this paper but since this construction is important in an other related problem ( $c f$. [13]) I shall briefly outline this generalization. If we denote by $\mathfrak{g}$ the Lie algebra of the simply connected group $G$ and if $\mathfrak{g}$ is assumed to be an NC-algebra then we can decompose as in Section $1.4 \mathfrak{g}=\mathfrak{n}_{R} \curlywedge\left(\mathfrak{q}_{R} \curlywedge \mathfrak{s}\right)$ and this gives the obvious semidirect product decomposition of the simply connected group $G$ associated to $\mathfrak{g}$

$$
G=N_{R} \curlywedge\left(Q_{R}<S\right),
$$

where $S$ is semisimple and simply connected. The lemma of Section 1.5 gives then the following:

Let $g_{1}, \ldots, g_{s} \in Q_{R} \curlywedge S$ and let us assume that $S$ is compact (in other words we are assuming that $G$ is amenable which was the hypothesis in [13]) let further

$$
\begin{gathered}
\quad\left|g_{j}\right| \leq u, \quad j=1, \ldots, s, \\
L_{k} \circ \kappa\left(g_{1} \cdots g_{i}\right) \leq \rho, \quad k=1, \ldots, \quad i=1,2, \ldots, s-1,
\end{gathered}
$$

where now $\kappa$ is the composition $(c f .[6])$

$$
Q_{R} \wedge S \rightarrow\left(Q_{R}<S\right) / N_{0} \cong\left(Q_{R} / N_{0}\right) \times S \rightarrow Q_{R} / N_{0} \cong Q / N \cong \mathbb{R}^{d} .
$$

The conclusion of the above hypothesis is then that the estimate (1.6.7) holds. The details will be left to the reader.

REMARK. Implicit in the considerations of this section is the definition of the "roots" for a general (not necessarily simply connected) soluble Lie group $G$. Indeed we have as above

$$
\text { Ad }: Q \rightarrow G L\left(\mathfrak{n}_{c}\right)
$$

where $\mathfrak{n} \subset \mathfrak{q}$ is the nilradical of the Lie algebra $\mathfrak{q}$ of $Q$. From the above we see that we can simultaneously triangulate Ad so that

$$
\operatorname{Ad}(q)=\left(\begin{array}{ccc}
\Xi_{1}(q) & & * \\
& \ddots & \\
\mathbf{0} & & \Xi_{n}(q)
\end{array}\right), \quad q \in Q
$$

where $\Xi_{j}: Q \rightarrow \mathbb{C}_{*}=\mathbb{C} \backslash\{0\}$ is a group homomorphism $\left(\mathbb{C}_{*}\right.$ has of course the multiplicative group structure). The above defines uniquely 
$\rho_{j}: Q \rightarrow \mathbb{R}$ and $\theta_{j}: Q \rightarrow \mathbb{T}=\mathbb{R}(\bmod 2 \pi),(j=1, \ldots, n)$ two group homomorphisms such that $\Xi_{j}(q)=\exp \left(\rho_{j}(q)+i \theta_{j}(q)\right)$ where clearly

$$
\rho_{j}(\operatorname{Exp}(x))=L_{j}(x), \quad x \in \mathfrak{q} .
$$

Using easy standard considerations (involving determinants) we can express the modular function

$$
m(q)=\frac{d^{r} q}{d^{\ell} q}=\exp \left(\sum_{j=1}^{n} \rho_{j}(q)\right) .
$$

One sees in particular that $G$ is unimodular if and only if $\operatorname{tr}\left(\operatorname{ad}_{\mathfrak{q}}(x)\right)=0$ $(x \in \mathfrak{q})$.

Finally just as before if $Q / N \cong V \times T$ where $N$ is the nilradical of $Q$ with $V \cong \mathbb{R}^{m}$ and $T \cong \mathbb{T}^{k}$ then the "roots" $\rho_{j}$ are defined on $V$ (i.e. $\left.\left.\rho_{j}\right|_{N} \equiv 1\right)$.

\subsection{Non amenable Lie algebras.}

In this section I shall consider $\mathfrak{g}$ a finite dimensional real Lie algebra and I shall denote by $\mathfrak{n} \subset \mathfrak{q} \subset \mathfrak{g}$ its radical and nilradical. The algebra $\mathfrak{g} / \mathfrak{q}$ is then semisimple or zero. Let us recall the following standard

Definition. We say that $\mathfrak{g}$ is amenable if $\mathfrak{g} / \mathfrak{q}$ is of compact type or zero. Otherwise we say that $\mathfrak{g}$ is non amenable.

Quite generally the Lie algebra $\mathfrak{g}$ can be written

$$
\mathfrak{g}=\mathfrak{q} \wedge \mathfrak{s},
$$

where $\mathfrak{s}$ is some Levi subalgebra $(c f .[6])$ and where of course $\mathfrak{s} \cong \mathfrak{g} / \mathfrak{q}$. When $\mathfrak{s} \neq 0$ we shall apply the Iwasawa decomposition on $\mathfrak{s}$ (cf. [9], [10], [14])

$$
\mathfrak{s}=\mathfrak{n}_{S}+\mathfrak{a}+\mathfrak{k},
$$

where $\mathfrak{n}_{S}$ is nilpotent and $\mathfrak{a}$ is abelian and normalizes $\mathfrak{n}_{S}$ so that $\mathfrak{n}_{S}+\mathfrak{a}$ is a soluble algebra. As for $\mathfrak{k}$ it is never 0 and it is the Lie algebra of some compact group. If $\mathfrak{g}$ is amenable we have $\mathfrak{n}_{S}=\mathfrak{a}=0$. Since $\mathfrak{s}$ normalizes $\mathfrak{q}$ it is clear that

$$
\mathfrak{r}=\mathfrak{q}+\mathfrak{n}_{S}+\mathfrak{a} \subset \mathfrak{g}
$$


is a soluble subalgebra of $\mathfrak{g}$ which I shall call an Iwasawa radical of $\mathfrak{g}$. The definition of $\mathfrak{r}$ is not "unique". In general several Iwasawa radicals exist in $\mathfrak{g}$. When $\mathfrak{g}$ is amenable $\mathfrak{q}$ is the only Iwasawa radical. Finally when $\mathfrak{s}=0$ and $\mathfrak{g}=\mathfrak{q}$ is soluble we shall agree to say that the Iwasawa radical of $\mathfrak{g}$ is $\mathfrak{r}=\mathfrak{q}=\mathfrak{g}$.

We can give now the following basic

Definition. We shall say that $\mathfrak{g}$ as above is a B-algebra (respectively $\mathrm{NB}$-algebra) if some Iwasawa radical of $\mathfrak{g}$ is a $\mathrm{C}$-algebra (respectively NC-algebra).

It is not obvious that a non amenable algebra cannot be both a $\mathrm{B}$ and an NB-algebra at the same time. But of course as we shall see this cannot be the case and the above definition gives a genuine classification on Lie algebras.

What is well known (but anything but trivial) is that if $\mathfrak{g}$ is semisimple of non compact type then it is an NB-algebra. This follows from the classification theorems that give the complete description of the reduced roots (i.e. the roots of the action of $\mathfrak{a}$ on $\mathfrak{n}_{S}$ ).

Let now $\mathfrak{q}=\mathfrak{q}_{1} \times \mathfrak{q}_{2}$ be the direct product two soluble algebras, and let $\mathfrak{n}=\mathfrak{n}_{1} \times \mathfrak{n}_{2}$ be the nilradical. It is clear that the set of real roots $L$ of $\mathfrak{q}$ can be identified with the set $\left(L_{1} \times\{0\}\right) \cup\left(\{0\} \times L_{2}\right) \subset V^{*}$ where $L_{i} \subset V_{i}^{*}=\left(\mathfrak{q}_{i} / \mathfrak{n}_{i}\right)^{*}, i=1,2$, are the real roots of $\mathfrak{q}_{i}(i=1,2)$ and $V=V_{1} \times V_{2}=\mathfrak{q} / \mathfrak{n}$. From this it follows that $\mathfrak{q}$ is an NC-algebra if and only if both $\mathfrak{q}_{1}$ and $\mathfrak{q}_{2}$ are.

Let now $\mathfrak{g}_{i}, i=1,2$, be two general Lie algebras and let $\mathfrak{g}=\mathfrak{g}_{1} \times \mathfrak{g}_{2}$. It is then clear that $\mathfrak{r} \subset \mathfrak{g}$ is an Iwasawa radical of $\mathfrak{g}$ if and only if $\mathfrak{r}=\mathfrak{r}_{1} \times \mathfrak{r}_{2}$ where $\mathfrak{r}_{i}$ is an Iwasawa radical of $\mathfrak{g}_{i}(i=1,2)$. From this it follows that (even without knowing that the above definition gives a classification) that $\mathfrak{g}$ is an NB-algebra if and only if $\mathfrak{g}_{1}$ and $\mathfrak{g}_{2}$ are both NB-algebras.

By the above definition, if $\mathfrak{g}$ is amenable then $\mathfrak{g}$ is a B-algebra (respectively NB-algebra) if and only if its radical $\mathfrak{q} \subset \mathfrak{g}$ is a C-algebra (respectively $\mathrm{NC}$-algebra). We also have

Proposition. Let $\mathfrak{g}$ be an arbitrary real Lie algebra, let $\mathfrak{q} \subset \mathfrak{g}$ be its radical. Let us assume that $\mathfrak{q}$ is a $\mathrm{C}$-algebra. Then $\mathfrak{g}$ is a $\mathrm{B}$-algebra.

Let us also state formally the classifying property of our definition. 
Classification. Let $\mathfrak{g}$ be an arbitrary real Lie algebra, then $\mathfrak{g}$ cannot be simultaneously $a \mathrm{~B}$ - and an NB-algebra.

The above classification is indirectly an automatic consequence of the main theorem of this paper. A direct algebraic proof can also be given. That algebraic proof does not seem to be very relevant for the rest of this paper and will therefore be deferred until the end of this chapter. The rest of this section will be devoted to the proof of the proposition.

Before I give the proof of the proposition, I shall have to examine more closely the Iwasawa radicals of the Lie algebra $\mathfrak{g}$. Let

$$
\mathfrak{r}=\mathfrak{q}+\mathfrak{n}_{S}+\mathfrak{a} \subset \mathfrak{g}
$$

be such an Iwasawa radical where I shall assume throughout in this section that $\mathfrak{g} / \mathfrak{q} \neq 0$ and let us denote by $\overline{\mathfrak{n}}=\mathfrak{n}+\mathfrak{n}_{S}$. We have then

Lemma. $\overline{\mathfrak{n}}$ is the nilradical of $\mathfrak{r}$.

Proof. I shall denote by $\mathfrak{n}_{r} \subset \mathfrak{r}$ the nilradical of $\mathfrak{r}$ and I shall prove first that

$$
\overline{\mathfrak{n}} \subset \mathfrak{n}_{r}
$$

To prove (1.7.1) observe first that $\mathfrak{n}$ is the nilradical of $\mathfrak{g}$ and therefore clearly $\mathfrak{n} \subset \mathfrak{n}_{r}$. We have on the other hand

$$
\mathfrak{n}_{S}=\left[\mathfrak{n}_{S}+\mathfrak{a}, \mathfrak{n}_{S}+\mathfrak{a}\right]
$$

This holds by the structure theory of semisimple algebras and the construction of the Iwasawa decompositions (cf. [9], [14, Proposition 5.10]). The conclusion is that

$$
\mathfrak{n}_{S}=\left[\mathfrak{n}_{S}+\mathfrak{a}, \mathfrak{n}_{S}+\mathfrak{a}\right] \subset[\mathfrak{r}, \mathfrak{r}] \subset \mathfrak{n}_{r}
$$

where the last inclusion holds because $\mathfrak{r}$ is soluble ( $f$. [6]). (1.7.1) follows.

Now $\mathfrak{n}_{r} \cap \mathfrak{q}$ is a nil-ideal of $\mathfrak{q}$ therefore $\mathfrak{n}_{r} \cap \mathfrak{q} \subset \mathfrak{n}$ (= the nilradical of $\mathfrak{q})$. It follows therefore from (1.7.1) that

$$
\mathfrak{n}_{r} \cap \mathfrak{q}=\mathfrak{n} .
$$


Let us now consider the projection

$$
\pi: \mathfrak{r} \rightarrow \mathfrak{r} / \mathfrak{q}=\mathfrak{n}_{S}+\mathfrak{a} .
$$

From general considerations it follows that $\pi\left(\mathfrak{n}_{r}\right)$ is a nilpotent ideal of $\mathfrak{r} / \mathfrak{q}$ and that therefore it lies inside the nilradical of $\mathfrak{r} / \mathfrak{q}=\mathfrak{n}_{S}+\mathfrak{a}$. That nilradical is exactly $\mathfrak{n}_{S}$ by (1.7.2) and the fact that, for all $0 \neq x \in$ $\left(\mathfrak{n}_{S}+\mathfrak{a}\right) \backslash \mathfrak{n}_{S},\left[x,\left[x,\left[\ldots\left[x, \mathfrak{n}_{S}\right] \ldots\right] \neq 0\right.\right.$. The conclusion is that

$$
\pi\left(\mathfrak{n}_{r}\right) \subset \mathfrak{n}_{S}
$$

and if we combine this with (1.7.4) we deduce that

$$
\mathfrak{n}_{r} \subset \mathfrak{n}+\mathfrak{n}_{S}=\overline{\mathfrak{n}}
$$

our lemma follows.

From the above lemma we see that we have the identification

$$
\mathfrak{r} / \mathfrak{n}_{r}=(\mathfrak{q} / \mathfrak{n})+\mathfrak{a}=V
$$

Let us now complexify $\mathfrak{n}_{c}=\mathfrak{n} \otimes \mathbb{C}, \overline{\mathfrak{n}}_{c}=\overline{\mathfrak{n}} \otimes \mathbb{C}$ and consider

$$
W=\mathfrak{n}_{c} /\left[\mathfrak{n}_{c}, \mathfrak{n}_{c}\right] \subseteq \bar{W}=\overline{\mathfrak{n}}_{c} /\left[\overline{\mathfrak{n}}_{c}, \overline{\mathfrak{n}}_{c}\right]
$$

The natural (induced by ad-) action of $V$ on $\bar{W}$ that was considered in Section 1.2 stabilizes $W$. Let us consider the root space decomposition

$$
W=W_{1} \oplus \cdots \oplus W_{m}
$$

with respect to the above action of $\mathfrak{q} / \mathfrak{n}(\subset V)$ on $W . \lambda_{1}, \ldots, \lambda_{m} \in$ $\operatorname{Hom}_{\mathbb{R}}(\mathfrak{q} / \mathfrak{n} ; \mathbb{C})$ are the corresponding roots. The important thing to observe is that (since $\mathfrak{q} / \mathfrak{n}$ and $\mathfrak{a}$ commute in $V$ !) each root space $W_{j}$ is stable by the action of $\mathfrak{a}(\subset V)$ and admits thus its proper root space decomposition

$$
W_{j}=W_{1}^{(j)} \oplus \cdots \oplus W_{m_{j}}^{(j)}
$$

under that action. The corresponding roots are $\rho_{1}, \ldots, \rho_{m_{j}} \in \operatorname{Hom}_{\mathbb{R}}$ $[\mathfrak{a} ; \mathbb{C}]$, (strictly speaking we need also a " $j$ " index and we should denote these roots by $\left.\rho_{i}^{(j)}=\rho_{i}, i=1,2, \ldots, m_{j}\right)$, and we have

$$
\sum_{i=1}^{m_{j}} \rho_{i}=0, \quad j=1, \ldots, m .
$$


To see (1.7.7) observe that the ad-action of $\mathfrak{q} / \mathfrak{n}$ on $W$ and the ad-action of $\mathfrak{s}$ on $W$ commute (since $[\mathfrak{s}, \mathfrak{q}] \subset[\mathfrak{g}, \mathfrak{q}] \subset \mathfrak{n}, c f$. [6]). It follows that the natural ad-action of $\mathfrak{a}$ on $W_{j}$ extends to a representation of the semisimple Lie algebra $\mathfrak{s}$ on $W_{j}$. The trace of such a representation is zero and (1.7.7) follows.

The very definition of (1.1.5) implies that

$$
\left(\operatorname{ad} x-\lambda_{j}(x)\right)^{N} \omega=0, \quad\left(\operatorname{ad} y-\rho_{s}(y)\right)^{N} \omega=0,
$$

where $x \in \mathfrak{q} / \mathfrak{n}, y \in \mathfrak{a}, \omega \in W_{s}^{(j)}$ (for $N$ large enough). Since the action of $\mathfrak{q} / \mathfrak{n}$ and of $\mathfrak{a}$ commute it follows that

$$
W=\sum_{j=1}^{m} \sum_{s=1}^{m_{j}} W_{s}^{(j)}
$$

is a root space decomposition of $W$ under the action of $V=(\mathfrak{q} / \mathfrak{n})+\mathfrak{a}$, and that the corresponding roots are

$$
\pi_{j, s}: V \text { э } x+y \longmapsto \lambda_{j}(x)+\rho_{s}(y), \quad x \in \mathfrak{q} / \mathfrak{n}, y \in \mathfrak{a} .
$$

(1.7.7) implies therefore that

$$
\sum_{s=1}^{m_{j}} \pi_{j, s}(x+y)=m_{j} \lambda_{j}(x), \quad j=1, \ldots, m, x \in \mathfrak{q} / \mathfrak{n}, y \in \mathfrak{a}
$$

Let us now, as in our proposition, make the assumption that $\mathfrak{q}$ is a $\mathrm{C}$-algebra and that there exists a non trivial representation of zero

$$
0=\sum_{j=1}^{m} \alpha_{j} \operatorname{Re} \lambda_{j}(x), \quad x \in \mathfrak{q} / \mathfrak{n}
$$

But (1.7.8) and (1.7.9) give then a non trivial representation of zero

$$
0=\sum_{j=1}^{m} \frac{\alpha_{j}}{m_{j}} \sum_{s=1}^{m_{j}} \operatorname{Re} \pi_{j, s}(v), \quad v \in V .
$$

The final step that is needed to complete proof of our proposition is that the $\pi_{j, s}$ 's can be identified to a subset of the roots of $\mathfrak{r}$ (in the sense of Section 1.1) i.e. referring to the action of $\mathfrak{r} / \mathfrak{n}_{r}$ on $\bar{W}$. This 
is of course easy by the obvious composition series argument and our proposition follows.

Observe that the converse of the above proposition does not hold. Indeed if $\mathfrak{s}$ is semisimple if $\mathfrak{q}$ is an R-algebra and if the semidirect product $\mathfrak{g}=\mathfrak{q} \wedge \mathfrak{s}$ is not direct then $\mathfrak{g}$ is in general a B-algebra. Indeed the trace of the action of $\mathfrak{s}$ on $\mathfrak{q}$ is zero.

\subsection{Unimodular Lie algebras.}

Let us recall that a finite dimensional Lie algebra $\mathfrak{g}$ is called unimodular if

$$
\operatorname{tr}\left(\operatorname{ad}_{\mathfrak{g}} x\right)=0, \quad x \in \mathfrak{g} .
$$

It follows at once that a unimodular Lie algebra that is in addition amenable is an NB-algebra if and only if it is an R-algebra. In this section we shall prove the following

Proposition. Let $\mathfrak{g}$ be a unimodular Lie algebra. Then either $\mathfrak{g}$ is a $\mathrm{B}$-algebra or $\mathfrak{g}$ is the direct product $\mathfrak{g}=\mathfrak{g}_{1} \times \mathfrak{s}$ where $\mathfrak{g}_{1}$ is an $\mathrm{R}$-algebra and $\mathfrak{s}$ is either 0 or semisimple.

The proof will be done in several steps. We shall assume that $\mathfrak{g}$ is not soluble and fix once and for all $\mathfrak{g}=\mathfrak{q} \curlywedge \mathfrak{s}$ and $\mathfrak{s}=\mathfrak{n}_{S}+\mathfrak{a}+\mathfrak{k}$ a Levi decomposition of $\mathfrak{g}$ and an Iwasawa decomposition of $\mathfrak{s}$. We shall assume as we may that $\mathfrak{s}$ is not compact. We have then

Lemma. Let $\mathfrak{g}, \mathfrak{q}, \mathfrak{n}_{S}, \mathfrak{a}$ be as above and let us assume that $\left[\mathfrak{q}, \mathfrak{n}_{S}+\mathfrak{a}\right]=$ $\{0\}$. Then $\mathfrak{g}$ can be written as a direct product $\mathfrak{g}=\mathfrak{g}_{1} \times \mathfrak{s}_{1}$ where $\mathfrak{g}_{1}$ is an $\mathrm{R}$-algebra and $\mathfrak{s}_{1}$ is either semisimple or $\{0\}$.

Proof. Indeed

$$
I=\{x \in \mathfrak{s}:[\mathfrak{q}, x]=0\} \subset \mathfrak{s}
$$

is an ideal in $\mathfrak{s}$ and since $\mathfrak{n}_{S}+\mathfrak{a} \subset I$ it follows that $\mathfrak{s}=I \times \tilde{\mathfrak{s}}$ where $\tilde{\mathfrak{s}}$ is either $\{0\}$ or a compact semisimple algebra. It suffices therefore to set $\mathfrak{g}_{1}=\mathfrak{q} 久 \tilde{\mathfrak{s}}$ and $\mathfrak{s}_{1}=I$ and our lemma follows.

Lemma. Let $\mathfrak{g}, \mathfrak{q}, \mathfrak{n}_{S}, \mathfrak{a}$ be as above and let us assume that $\left[\mathfrak{n}, \mathfrak{n}_{S}+\right.$ $\mathfrak{a}]=\{0\}$ where $\mathfrak{n} \subset \mathfrak{q}$ is as before the nilradical of $\mathfrak{g}$. We have then $\left[\mathfrak{q}, \mathfrak{n}_{S}+\mathfrak{a}\right]=\{0\}$. 
Proof. The semisimple algebra $\mathfrak{s}$ acts by ad on $\mathfrak{q}$ and stabilizes the subspace $\mathfrak{n}$. By H. Weyl's theorem therefore ( $c f$. [5], [6]) we can find a direct complement $\mathfrak{q}=\mathfrak{n} \oplus \mathfrak{l}$ such that $[\mathfrak{s}, \mathfrak{l}] \subset \mathfrak{l}$. Since on the other hand we also have $[\mathfrak{s}, \mathfrak{q}] \subset \mathfrak{n}$ it follows that $[\mathfrak{s}, \mathfrak{l}]=\{0\}$ and therefore for all $x \in \mathfrak{s},[x, \mathfrak{q}] \subset[x, \mathfrak{n}]$ and our lemma follows.

Let us now consider the ad-action of $\mathfrak{r}$ on $\overline{\mathfrak{n}}=\mathfrak{n}+\mathfrak{n}_{S}=\mathfrak{n}_{r}$ the nilradical of $\mathfrak{r}$ ( $c f$. Section 1.7). It clearly stabilizes $\mathfrak{n}$ and, $\mathfrak{r}$ being soluble, a basis can be chosen on $\mathfrak{n}_{c}$ for which the adjoint action takes the form

$$
\operatorname{ad}_{\mathfrak{n}_{c}}(x)=\left(\begin{array}{ccc}
\lambda_{1}(x) & & * \\
& \ddots & \\
\mathbf{0} & & \lambda_{p}(x)
\end{array}\right), \quad x \in \mathfrak{r} .
$$

since $\operatorname{ad}_{\mathfrak{n}}(x)$ is nilpotent for every $x \in \overline{\mathfrak{n}}$ it follows that we can identify each $\lambda_{j} \in \operatorname{Hom}_{\mathbb{R}}[V ; \mathbb{C}]$ where as in $(1.7 .5) V=\mathfrak{r} / \overline{\mathfrak{n}}=(\mathfrak{q} / \mathfrak{n})+\mathfrak{a}$. We have then

\section{Lemma.}

i) All the $\lambda_{j}$ above are real valued on $\mathfrak{a}$, i.e.

$$
\lambda_{j}(x) \in \mathbb{R}, \quad j=1, \ldots, p, x \in \mathfrak{a} .
$$

ii) The trace is zero on $\mathfrak{a}$, i.e.

$$
\operatorname{tr}\left(\operatorname{ad}_{\mathfrak{n}} x\right)=\sum_{j=1}^{p} \lambda_{j}(x)=0, \quad x \in \mathfrak{a} .
$$

iii) If we assume that $\left[\mathfrak{n}, \mathfrak{n}_{S}+\mathfrak{a}\right] \neq\{0\}$, then there exists a $j(1 \leq$ $j \leq p)$, say $j=1$, for which $\lambda_{1}(x) \neq 0$ for some $x \in \mathfrak{a}$.

We shall defer the proof of the lemma until later and complete the proof of the proposition, assuming as we may because of our first two lemmas, that $\left[\mathfrak{n}, \mathfrak{n}_{S}+\mathfrak{a}\right] \neq\{0\}$.

The unimodularity of $\mathfrak{g}$ implies the unimodularity of the algebra $\mathfrak{q}$ which says that

$$
\operatorname{tr}\left(\operatorname{ad}_{\mathfrak{n}}(x)\right)=\sum_{j=1}^{p} \lambda_{j}(x)=0, \quad x \in \mathfrak{q} / \mathfrak{n} .
$$


Part ii) of our previous lemma implies therefore that

$$
\operatorname{tr}\left(\operatorname{ad}_{\mathfrak{n}}(x)\right)=\sum_{j=1}^{p} \lambda_{j}(x)=0, \quad x \in V=\mathfrak{q} / \mathfrak{n}+\mathfrak{a}
$$

Since on the other hand by i) and iii) of our previous lemma and our hypothesis we have $\operatorname{Re} \lambda_{1}=L_{1} \neq 0,(1.8 .1)$ says that $\mathfrak{r}$ is a C-algebra. By definition therefore $\mathfrak{g}$ is a B-algebra and the proof of our proposition is complete. (As in the end of Section 1.7 we have to use a standard composition series argument to verify that the non zero among the $\operatorname{Re} \lambda_{j}$ 's can be identified to real roots of $\mathfrak{r}$ ).

It remains to give the proof of the last lemma.

Proof OF ii). This uses the same argument as in Section 1.7. Indeed the action of $\mathfrak{a}$ on $\mathfrak{n}$ extends to an action of $\mathfrak{s}$ on $\mathfrak{n}$ i.e. to a representation of a semisimple algebra and therefore has trace equal to zero.

To see parts i) and iii) of the lemma we start from the following construction:

Let $\mathfrak{g}$ be some real semisimple algebra and let $\mathfrak{u} \subset \mathfrak{g}$ be some real subalgebra that is a semisimple algebra of compact type. Let further

$$
\theta=\mathfrak{g} \longmapsto \mathfrak{g l}_{n}(\mathbb{C})
$$

a real algebra homomorphism. Then there exists $\langle\cdot, \cdot\rangle$ some Hermitian product on $\mathbb{C}^{n}$ that is invariant under $\mathfrak{u}$, i.e.

$$
\left\langle\theta(x) z_{1}, z_{2}\right\rangle+\left\langle z_{1}, \theta(x) z_{2}\right\rangle=0, \quad x \in \mathfrak{u}, z_{1}, z_{2} \in \mathbb{C}^{n}
$$

The proof of this is of course very easy. Indeed let $G$ be the simply connected semisimple group that corresponds to $\mathfrak{g}$ and let $U \subset G$ be the (compact) subgroup that corresponds to $\mathfrak{u}$. $\theta$ induces then

$$
\theta: U \longrightarrow G L_{n}(\mathbb{C})
$$

and, since for any non singular matrix $M \in M_{n \times n}(\mathbb{C})\langle z, u\rangle_{M}=$ $\langle M z, M u\rangle$ is a new Hermitian product on $\mathbb{C}^{n}$, the Hermitian product

$$
\langle\langle z, u\rangle\rangle=\int_{U}\langle\theta(x) z, \theta(x) u\rangle d x
$$


is invariant under the action of $U$. Taking the differential we obtain (1.8.2).

The above observation has to be combined with the fact that for the Iwasawa decomposition $\mathfrak{s}=\mathfrak{n}_{S}+\mathfrak{a}+\mathfrak{k}$ if we complexify $\mathfrak{s}_{c}$ we can write $\mathfrak{s}_{c}=\mathfrak{k}_{0} \oplus \mathfrak{p}_{0}$ in such a way that $\mathfrak{u}=\mathfrak{k}_{0}+i \mathfrak{p}_{0}$ is a compact real semisimple subalgebra of the (underlying) real algebra $\mathfrak{s}_{c}$, and we can do so in such a way that $\mathfrak{a} \subset \mathfrak{p}_{0}$. This fact is of course anything but obvious but in some sense it is the very basis of the construction of the Iwasawa decomposition (cf. [9], [14]).

This being said, we see, that if we let $\mathfrak{g}$ in (1.8.2) to be the underlying real algebra of $\mathfrak{s}_{c}$, there exists $\langle\cdot, \cdot\rangle$ some Hermitian product on $\mathbb{C}^{n}$ for which all the matrices $\theta(x)\left(x \in i \mathfrak{p}_{0}\right)$ are skew-Hermitian. All the matrices $\theta(x)\left(x \in \mathfrak{a} \subset \mathfrak{p}_{0}\right)$ are therefore Hermitian. It follows that all the matrices $\theta(x)(x \in \mathfrak{a})$ have real eigenvalues and if all the eigenvalues of $\theta(x)$ are zero then $\theta(x)=0$.

If we apply this last observation to the representation of $\mathfrak{s} \otimes \mathbb{C}$ on $\mathfrak{n} \otimes \mathbb{C}$ induced by the adjoint action of $\mathfrak{s}$ on $\mathfrak{n}$ the assertions i) and iii) of the lemma follows.

I shall finish this section with an example that shows that unimodularity is essential for the above proposition to hold. I shall consider the 2-dimensional group of "affine motions" which is the Lie group

$$
G=\mathbb{R}^{2} \curlywedge\left(\mathbb{R} \times S L_{2}(\mathbb{R})\right)=\left(\mathbb{R}^{2} \curlywedge \mathbb{R}\right) \curlywedge S L_{2}(\mathbb{R}),
$$

where $\mathbb{R}$ acts on $\mathbb{R}^{2}$ by dilatation (i.e. $x \mapsto e^{\rho} x, x \in \mathbb{R}^{2}, \rho \in \mathbb{R}$ ) and $S L_{2}(\mathbb{R})$ acts on $\mathbb{R}^{2}$ by the natural action (of course $\mathbb{R}$ and $S L_{2}(\mathbb{R})$ commute in (1.8.3)). The third term in (1.8.3) is of course a Levi decomposition of $G$ and $G$ is not unimodular since the radical $Q=$ $\mathbb{R}^{2} \curlywedge \mathbb{R}$ is not unimodular. It is clear of course that $\mathfrak{g}$ the Lie algebra of $G$ is not of the form $\mathfrak{g}_{1} \times \mathfrak{s}$ as in our proposition. The above algebra $\mathfrak{g}$ however is an NB-algebra and therefore, by the classification in Section 1.7 , it is not a B-algebra.

Indeed with the "standard" Iwasawa decomposition of $S L_{2}(\mathbb{R})$ and the corresponding Iwasawa radical $\mathfrak{r}$ obtained by the Levi decomposition (1.8.3) we have, with our previous notations,

$$
\mathfrak{r} / \overline{\mathfrak{n}}=V=R+\mathfrak{a} \cong \mathbb{R}^{2} .
$$

Here $\mathfrak{a} \cong \mathbb{R}$ is the $\mathfrak{a}$ component of the Iwasawa decomposition of $S L_{2}(\mathbb{R})$ and $R \cong \mathbb{R} \cong \mathfrak{q} / \mathfrak{n}$. The action of $R$ on $\mathbb{R}^{2}$ is of course given by 
dilatation. This means that the two roots $\lambda_{1}, \lambda_{2}$ of $\mathfrak{q}$ (in the sense of sections 1.1 and 1.2) are real and $\lambda_{1}(x)=\lambda_{2}(x) \neq 0$ if $0 \neq x \in R$. The algebra $\mathfrak{r}$ is therefore an NC-algebra and our assertion follows.

\subsection{The uniqueness of the Iwasawa radical and an intrinsic definition.}

In this section I shall prove the following

Proposition. Let $\mathfrak{g}$ be a real Lie algebra and let $\mathfrak{r}_{1}, \mathfrak{r}_{2}$ two Iwasawa radicals of $\mathfrak{g}$. Then there exists $\alpha \in \operatorname{Int}(\mathfrak{g})$ such that $\alpha\left(\mathfrak{r}_{1}\right)=\mathfrak{r}_{2}$.

This proposition is not essential for the rest of this paper but it does help to give an intrinsic status to the notions introduced in the previous sections. The proof is an inmediate consequence of the following sequence of well known, but highly non trivial, facts:

1) Let $\mathfrak{g}=\mathfrak{q}<\mathfrak{s}_{1}=\mathfrak{q}<\mathfrak{s}_{2}$ be two Levi decompositions of $\mathfrak{g}$ then there exists $\alpha: \mathfrak{g} \rightarrow \mathfrak{g}$ an inner automorphism of $\mathfrak{g}$ such that $\alpha\left(\mathfrak{s}_{1}\right)=\mathfrak{s}_{2}$ (cf. [6, Theorem 3.14.2]).

Let now $\mathfrak{s}_{i}=\mathfrak{k}_{i}+\mathfrak{p}_{i}, i=1,2$ be Cartan decomposition of the above two semisimple algebras. By composing, if necessary, the automorphism $\alpha \in \operatorname{Int}(\mathfrak{g})$ by an appropiate element of $\operatorname{Int}\left(\mathfrak{s}_{i}\right)$ we can then assume in addition the following fact (cf. [9, Theorem 7.2] of the first edition):

2) The inner automorphism $\alpha$ is such that

$$
\alpha\left(\mathfrak{k}_{1}\right)=\mathfrak{k}_{2}, \quad \alpha\left(\mathfrak{p}_{1}\right)=\mathfrak{p}_{2} .
$$

Let now $\mathfrak{a}_{i} \subset \mathfrak{p}_{i}, i=1,2$ be a maximal abelian subalgebra and let

$$
\mathfrak{s}_{i}=\mathfrak{k}_{i}+\mathfrak{a}_{i}+\mathfrak{n}_{i}, \quad i=1,2,
$$

the Iwasawa decompositions that correspond to these choices of $\mathfrak{a}_{i}$ and to some choice of $\Sigma_{+}^{i} \subset \mathfrak{a}_{i}^{*}\left(=\operatorname{Hom}_{\mathbb{R}}\left(\mathfrak{a}_{i} ; \mathbb{R}\right)\right)$ the positive restricted roots on $\mathfrak{a}_{i}$ (i.e. the finitely many choices of the corresponding Weyl chambers). By the standard facts concerning the Iwasawa decomposition we see therefore that we can further compose the $\alpha \in \operatorname{Int}(\mathfrak{g})$ by an appropiate element in $\operatorname{Int}\left(\mathfrak{k}_{i}\right)$ and guarantee the following additional fact (cf. [14, Section 5.13 and Corollary 5.18]): 
3) The inner automorphism $\alpha \in \operatorname{Int}(\mathfrak{g})$ is such that

$$
\alpha\left(\mathfrak{k}_{1}\right)=\mathfrak{k}_{2}, \quad \alpha\left(\mathfrak{a}_{1}\right)=\mathfrak{a}_{2}, \quad \alpha\left(\mathfrak{n}_{1}\right)=\mathfrak{n}_{2} .
$$

The final conclusion clearly is that $\alpha\left(\mathfrak{r}_{1}\right)=\mathfrak{r}_{2}$ and this proves our proposition.

I shall finish up this section by giving, without proofs, what amounts to an alternative, more intrinsic, but less manageable, definition of the Iwasawa radical. Let $\mathfrak{g}$ be an arbitrary real Lie algebra and let $\mathfrak{l} \subset \mathfrak{g}$ be a amenable subalgebra, such that for some Lie group $G$ that corresponds to $\mathfrak{g}, \mathfrak{l}$ corresponds to a closed subgroup $L$ such that $G=L \cdot K$ where $K$ is a compact subgroup. We can then show that $\mathfrak{l}$ is a C- (respectively NC-) algebra if and only if $\mathfrak{g}$ is a B- (respectively NB) algebra. This, among other things is a consequence of the analytic theory developed in this paper. The Iwasawa radical clearly has the above property. Other examples of such subalgebras are $\mathfrak{l}=\pi^{-1}$ (a minimal boundary subalgebra of $\mathfrak{g} / \mathfrak{q}$ ) with $\pi: \mathfrak{g} \rightarrow \mathfrak{g} / \mathfrak{q}$ the canonical map and the standard terminology of semi-simple groups ( $f f . \quad[26]$ ). Such subalgebras will be called minimal boundary subalgebras of $\mathfrak{g}$.

We have in particular (the proof will be left as an exercise for the reader) the following

Proposition. All the minimal boundary subalgebras of $\mathfrak{g}$ are conjugate (under: $\operatorname{int}(\mathfrak{g})$ ) in $\mathfrak{g}$. The algebra $\mathfrak{g}$ is a $\mathrm{B}$-algebra if the minimal boundary subalgebras are $\mathrm{C}$-algebras (i.e. if their radicals are $\mathrm{C}$-algebras, $c f$. [13]).

Presented like this the B-NB classification becomes "subordinate" to the C-NC classification of the amenable algebras. "Philosophically" what the theorems of this paper say is that for non amenable groups the principal term $e^{-\lambda t}$ of the heat kernel $\phi_{t}$ comes from the "spectral gap" and that the error term $e^{\lambda t} \phi_{t}(g)$ is controlled by the geometry of the minimal boundary subgroups. 


\section{Basic geometric structure.}

\subsection{The Haar measure.}

Let $G$ be a locally compact group and let $Z \subset K \subset G, R \subset G$ be closed subgroups such that $G=R K$ and $K / Z$ is compact and $Z \subset G$ central. Quite generally we shall denote throughout by $d h=d^{\ell} h$ and by $d^{r} h$ the left and the right Haar measures of the locally compact group $H$. Among the above groups $K$ is unimodular and $d k=d^{r} k=d^{\ell} k$.

The example to keep in mind is the Iwasawa decomposition of a connected real semisimple group $S=N A K$ where $R=N A$ and $Z$ is the discrete center of $S$. More generally when $G$ is a simply connected real Lie group then we can write $G=Q \curlywedge S$ where $Q$ is the radical and $S=N A K$ is a semisimple simply connected Levi subgroup. We can set $G=R K$ with $R=Q<N A$. Indeed if $Z(K) \subset K$ is the discrete center of $S$ then it is well known and easy to prove ( $c f$. [15] for a proof) that there exists $Z \subset Z(K)$ of finite index (i.e. $[Z(K): Z]<+\infty$ ) such that $Z$ is central in $G$ (when $\mathfrak{q}$ is the Lie algebra of $Q$ this here amounts to saying that $\left.\operatorname{Ad}(Z)\right|_{\mathfrak{q}}=$ identity). We have

Lemma. Let $G, R, K, Z$ be as above and assume that $R \cap K=\{e\}$. Then

$$
\int_{G} f(g) d^{\ell} g=\int_{R \times K} f(r k) d^{\ell} r d k
$$

for an appropiate normalization of the Haar measures.

REMARK. In the above lemma we can relax the conditions that $K / Z$ is compact and $Z$ is central and impose instead the unique condition that the modular function $m(g)$ on $G$ satisfies $m(k) \equiv 1$ for all $k \in K$. One can also refer to $[35,1.5 .1]$ or to $[9],[25$, Chapter 7 , Section 2, number 9] for analogous and more general results.

Proof. There exists a unique $\Phi(r, k)>0(r \in R, k \in K)$ such that

$$
\int_{G} f(g) d^{\ell} g=\int_{R \times K} \Phi(r, k) f(r k) d^{\ell} r d k
$$

$\Phi$ is just the Jacobian of the mapping $R \times K \rightarrow G((r, k) \rightarrow r k)$.

The uniqueness of the above $\Phi$ and the left invariance of $d^{\ell} g$ implies that

$$
\Phi\left(r_{1} r, k\right)=\Phi(r, k)=\Phi_{0}(k), \quad r, r_{1} \in R, k \in K
$$


with a new function $\Phi_{0}>0$ on $K$. If we use the right action $g \mapsto g k$ $(k \in K)$ on $d^{\ell} g$ we see that it sends $d^{\ell} g$ to $m(k) d^{\ell} g$ where $m(\cdot)$ is the modular function on $G$. By our hypothesis $m(k)=1$ and therefore $\Phi_{0}\left(k k_{1}\right)=\Phi_{0}(k)\left(k, k_{1} \in K\right)$ and $\Phi_{0}$ is a constant.

We shall introduce now a basic notation that will be used throughout this paper.

i) $R$ will denote some locally compact group assigned with its $d r=$ $d^{\ell} r$ and $d^{r} r$ measure. In practice $R$ will always be a Lie group and more often than not a soluble Lie group.

ii) $K$ will be some $C^{\infty}$ manifold asigned with some $C^{\infty}$ non vanishing measure $d k$. More generally $K$ could be an abstract measure space.

iii) For any measure $\tilde{d} r$ on $R$ we shall consider the measure $\tilde{d} x=$ $\tilde{d} r \otimes d k$ on $X=R \times K$ (the product space rather than group product). We shall denote, in particular

$$
d x=d^{\ell} x=d^{\ell} r \otimes d k, \quad d^{r} x=d^{r} r \otimes d k .
$$

iv) More often than not I shall assume that there exists $Z$ some discrete group acting (discretely) on $K$ stabilizing $d k$ and such that $K / Z$ is a $C^{\infty}$ manifold.

v) For our applications $K$ as in iv) will be a locally compact (more often than not a Lie) group and $d k$ will be the Haar measure, $Z \subset K$ will then be some discrete central subgroup.

vi) We shall say that we are in the "group case" $X=G$ if $G$ is a locally compact group (more often than not a connected Lie group) and if $R, K$ are closed subgroups such that $R \cap K=\{e\}$ and such that the conditions of the above lemma are verified. We set then $X=R \times K$ which we identify as a measure space, or even as a $C^{\infty}$-manifold, with G.

The above construction admits a number of useful generalizations which although not essential for us are worth noting. For instance, in practice we can often write a connected Lie group in the form $G=R K$ where $R$ is closed (but not necessarily connected) and $K$ is an analytic subgroup (but not neccesarily closed) and such that $Z=R \cap K$ is a (closed) discrete central subgroup of $G$. The general Levi decomposition of $G$ is of the above form. We can then identify $Z$ to a closed central subgroup of the Lie group $K$ (for its intrinsic analytic structure) and 
consider the projection $\pi: K \rightarrow K / Z$ if $\Sigma \subset K$ is some Borel section of $\pi$ (i.e. $\pi$ is (1-1) from $\Sigma$ onto $K / Z$ ), we shall denote by $(\sigma)=\pi^{-1} \sigma \in$ $\Sigma \subset G, \sigma \in K / Z$. We can then identify $G$ with $R \times K / Z$ by the mapping

$$
(r, \sigma) \longrightarrow r(\sigma)=g
$$

For the above section it is clear that

$$
\left(\sigma_{1} \sigma_{2}\right)=r\left(\sigma_{1}\right)\left(\sigma_{2}\right), \quad\left(\sigma_{1}^{-1}\right)=\tilde{r}\left(\sigma_{1}\right)^{-1},
$$

where $r, \tilde{r} \in Z$. From these relations it immediately follows that the proof of the above lemma generalizes and that the above identification identifies $d^{\ell} r \otimes d_{K / Z} \sigma$ with $d^{\ell} g$ provided of course that $m_{G}(k)=1$ $(k \in K)$.

\subsection{The left invariant operators.}

$X$ will be here as in Section 2.1 and we shall examine positive $R$-left invariant operators on $X$

$$
\left\{\begin{array}{l}
T: C_{0}^{\infty}(X) \longrightarrow C^{\infty}(X) ; \quad T f \geq 0, \text { for all } f \geq 0, \\
T\left(f_{r}\right)=(T f)_{r} ; f_{r}\left(r_{1}, k\right)=f\left(r r_{1}, k\right), r, r_{1} \in R, k \in K
\end{array}\right.
$$

Let $\mu_{h, k} \in M(R)(h, k \in K)$ be a family of positive measure (more often than not I shall assume that they are bounded measures) and let $L(h, d k)$ be some positive "kernel" on $K(e . g . \quad L(h, d k)=L(h, k) d k$ where $L(h, k) \geq 0$ but of course more general kernels could be considered). An invariant operator as in (2.2.1) can then be defined by the formula

$$
\begin{aligned}
T f(r, h) & =\int_{K} L(h, d k)\left(f(\cdot, k) * \mu_{h, k}\right)(r) \\
& =\int_{K} L(h, d k) \int_{R} f\left(r r_{1}^{-1}, k\right) d \mu_{h, k}\left(r_{1}\right), \quad f \geq 0,
\end{aligned}
$$

provided that $L$ and the $\mu$ 's satisfy the appropiate smoothness conditions. If in particular $f=\varphi \otimes \psi, \varphi \in C_{0}(R), \psi \in C_{0}(K)$ we have

$$
T f(r, h)=\int L(h, d k)\left(\varphi * \mu_{h, k}(r)\right) \psi(k)
$$


Motivated by this we shall introduce the notation

$$
\begin{gathered}
L f(h)=\int_{K} L(h, d k) f(k), \quad f \in C_{0}(K), \\
T=L \otimes\{* \mu\}=L(h, d k) \otimes\left\{* \mu_{h, k}\right\} .
\end{gathered}
$$

The representation (2.2.3) of $T$ is clearly not unique (e.g. replace $\left.L \rightarrow \alpha(h) L ; \mu_{h, k} \rightarrow \alpha^{-1}(h) \mu_{h, k}\right)$. We shall say that the representation (2.2.3) is normal if $\mu_{h, k} \in \mathbb{P}(R)$ is a probability measure for each $h, k \in K$. It is clear that under obvious (and reasonable) conditions a positive $R$-left invariant operator admits a unique normal representation as in (2.2.3). To see the uniqueness observe that for a normal representation we have

$$
L f=g, f \in C_{0}^{\infty}(k) \quad \text { if and only if } \quad T(f \otimes \mathbf{1})=g \otimes \mathbf{1}
$$

with $\mathbf{1}(r)=1(r \in R)$. For normal representations it follows in particular from (2.2.4) that $T$ is markovian (respectively sub-markovian) i.e. that $T 1=1$ (respectively $T 1 \leq 1$ ) if and only if $L$ is markovian (respectively sub-markovian).

Let finally $\left(T_{j} j=1,2, \ldots\right)$ be a sequence of positive $R$-left invariant operators on $X$ as above. We can then define the $R$-left invariant (time inhomogeneous in general) Markov chain $\left(x_{n} \in X n=1,2, \ldots\right)$ by the condition that $T_{j} j=2,3, \ldots$ are the transition operators

$$
T_{j} f(x)=\int \mathbb{P}\left[x_{j} \in d y / / x_{j-1}=x\right] f(y) .
$$

\subsection{The group case and the convolution operators.}

We shall consider here $G$ a locally compact group and $d \mu(g)=$ $\varphi(g) d g=\psi(g) m(g) d g(g \in G)$ some positive measure where $m$ is the modular function. Let $T$ be the corresponding convolution operator

$$
T f(g)=f * \mu(g)=\int_{G} f\left(g g_{1}^{-1}\right) d \mu\left(g_{1}\right)=\int_{G} f\left(g g_{1}^{-1}\right) \varphi\left(g_{1}\right) d g_{1}
$$

In this section we shall also assume that we are in the group case $X=G=R K$ as in vi) of Section 2.1 , and we shall analyze the $R$ left invariant operator $T$ on $X$. 
We shall adopt the notation

$$
g=r k, \quad g_{i}=r_{i} k_{i}, \quad i=1,2, \quad r, r_{i} \in R, \quad k, k_{i} \in K,
$$

and by the Lemma in Section 2.1 we have

$$
T f(r k)=\iint_{R \times K} f\left(r k k_{1}^{-1} r_{1}^{-1}\right) \varphi\left(r_{1} k_{1}\right) d r_{1} d k_{1}
$$

Let us fix $k \in K$ and consider the (1-1) correspondence $\left(r_{1}, k_{1}\right) \leftrightarrow$ $\left(r_{2}, k_{2}\right)$ given by

$$
g=r_{1} k_{1}=k_{2}^{-1} r_{2} k, \quad d g=d r_{1} d k_{1}=J\left(r_{2} ; k_{2}, k\right) d r_{2} d k_{2},
$$

where $J(\cdot ; \cdot, k)$ is of course the Jacobian. We have thus

$$
T f(r k)=\iint_{R \times K} f\left(r r_{2}^{-1} k_{2}\right) \varphi\left(k_{2}^{-1} r_{2} k\right) J\left(r_{2} ; k_{2}, k\right) d r_{2} d k_{2} .
$$

We have

Lemma. The Jacobian $J\left(r ; k^{\prime}, k\right)=J(r)$ is independent of $k, k^{\prime} \in K$ and

$$
J(r)=\frac{m_{R}(r)}{m_{G}(r)}, \quad r \in R,
$$

where $m_{R}(\cdot)$ is the modular function of $R$ amd $m_{G}(\cdot)$ is the modular function of $G$.

Proof. By the unimodularity of $K$ we have (with obvious notations)

$$
d k=d k^{-1}=d\left(k_{0} k^{ \pm 1}\right)=d\left(k^{ \pm 1} k_{1}\right), \quad k_{0}, k_{1} \in K .
$$

This and the definition (2.3.2) imply that $J\left(r ; k_{0} k^{\prime}, k k_{1}\right)=J\left(r ; k^{\prime}, k\right)$ for all $k, k^{\prime}, k_{0}, k_{1} \in K$ (it is only a matter of testing $\int f(g) d g=$ $\int f\left(k_{2}^{-1} r_{2} k\right) J\left(r_{2} ; k_{2} k\right) d r_{2} d k_{2}$ on $f_{h}(\cdot)=f(h \cdot)$ and on $\left.f^{h}(\cdot)=f(\cdot h)\right)$. The first part of the lemma follows and (2.3.2) takes the form

$$
d g=d r_{1} d k_{1}=J\left(r_{2}\right) d r_{2} d k_{2}, \quad g=r_{1} k_{1}=k_{2}^{-1} r_{2} k .
$$

Observe now that since $d^{r} g=d g^{-1}, d^{r} r=d r^{-1}, d k=d k^{-1}$ the lemma of Section 2.1 implies that with the parametrization $g=k r(k \in K$, 
$r \in R$ ) we have $d^{r} g=d^{r} r d k$ which together with (2.3.5) allows us to conclude that

$$
\begin{aligned}
d^{r} g & =d k_{2} d^{r} r_{2}=d k_{2} m_{R}\left(r_{2}\right) d r_{2} \\
& =m_{G}(g) d g=m_{G}(g) J\left(r_{2}\right) d r_{2} d k_{2}, \quad g=k_{2}^{-1} r_{2} k
\end{aligned}
$$

(2.3.4) follows.

If we use the above lemma in (2.3.3) we finally obtain

$$
\left\{\begin{array}{l}
T f(r k)=\iint f\left(r r_{1}^{-1} k_{1}\right) M\left(r_{1} ; k_{1}, k\right) d r_{1} d k_{1}, \\
M\left(r ; k_{1}, k\right)=\varphi\left(k_{1}^{-1} r k\right) \frac{m_{R}(r)}{m_{G}(r)} \\
\quad=\psi\left(k_{1}^{-1} r k\right) m_{R}(r), \quad r \in R, k, k_{1} \in K, \\
T f(r k)=\iint f\left(r r_{1}^{-1} k_{1}\right) \psi\left(k_{1}^{-1} r_{1} k\right) d^{r} r_{1} d k_{1} .
\end{array}\right.
$$

The $K$-bi-invariant case deserves special attention. We say that the operator (2.3.1) is $K$-bi-invariant if $(T f)^{k}=T f^{k}\left(k \in K, f^{k}(g)=\right.$ $f(g k))$. Clearly this is the case if and only if the inner automorphism $I_{k}$ : $G \rightarrow G, I_{k}: g \rightarrow k^{-1} g k$ stabilizes the measure $\mu$. By our hypothesis $d g$ is also stable by the action of $I_{k}$. Therefore it follows that $\psi\left(k x k^{-1}\right)=$ $\psi(x)$ and we can write (2.3.6) in the form

$$
\begin{aligned}
T f(r k) & =\iint f\left(r r_{1}^{-1} k_{1}^{-1} k\right) \psi\left(r_{1} k_{1}\right) d^{r} r_{1} d k_{1} \\
& =\iint f\left(r r_{1}^{-1} k_{1}\right) \psi\left(k k_{1}^{-1} r_{1}\right) d^{r} r_{1} d k_{1},
\end{aligned}
$$

i.e. as a convolution on the product group $R \times K$.

The point of a $K$-bi-invariant operator in the above context is that it can be identified with an operator on the homogeneous space $G / K=\{g K: g \in G\}$. We can then identify $G / K$ with $R$ and since left translation by elements of $R$ clearly commutes with the projection $G \rightarrow G / K \cong R$ the operator thus obtained on $R$ is a convolution operator

$$
f \longmapsto f * \tilde{\mu}, \quad f \in C_{0}(R),
$$


where $\tilde{\mu} \in M(R)$. From (2.3.7) we see that we have in fact $d \tilde{\mu}(r)=$ $\tilde{\varphi}(r) d^{r} r$ with

$$
\tilde{\varphi}(r)=\int_{K} \varphi(r k) d k, \quad r \in R
$$

The above two formulas (2.3.8) and (2.3.9) are not used in this paper but have the merit of putting the above notions in the correct "perspective" and are relevant in the "semisimple theory" which will be developed elsewhere.

2.4. The composition, the adjoint, $\|\cdot\|_{p \rightarrow p}$ norms, amenability and the "local" estimate.

Let us consider

$$
T_{i}=L_{i}(h, d k) \otimes\left\{* \mu_{h, k}^{(i)}\right\}, \quad i=1,2, \ldots,
$$

a sequence of $R$-left invariant operators on $X$ as in Section 2.2. It is clear then that

$$
\begin{array}{r}
T_{1} \circ \cdots \circ T_{n}=\int_{k_{1} \in K} \cdots \int_{k_{n-1} \in K} L_{1}\left(h, d k_{1}\right) \cdots L_{n}\left(k_{n-1}, d k\right) \\
\otimes\left\{*\left(\mu_{h, k_{1}}^{(1)} * \cdots * \mu_{k_{n-1}, k}^{(n)}\right)\right\}
\end{array}
$$

with obvious notations. To simply notations let $L(h, d k)=L(h, k) d k$ and let

$$
T=L(h, k) d k \otimes\left\{* \mu_{h, k}\right\}
$$

as in (2.2.3, Section 2.2). Let then $T^{*}$ be the formal adjoint operator with respect to $d^{r} x=d^{r} r \otimes d k$ then clearly

$$
T^{*}=L^{*}(h, k) d k \otimes\left\{* \mu_{h, k}^{*}\right\},
$$

where $L^{*}(h, k)=L(k, h)$ and $\mu_{h, k}^{*}=\check{\mu}_{k, h}$ where for any measure $\nu$ on $R$ we adopt throughout the notation $d \check{\nu}(g)=d \nu\left(g^{-1}\right)$ (i.e. $\check{\nu}$ is the image of $\nu$ under the mapping $g \mapsto g^{-1}$ on $R$ ). This follows trivially from the fact that the formal adjoint with respect to $d^{r} r$ of the operator $f \mapsto f * \nu, f \in C_{0}(R), \nu \in M(R)$ is $f \mapsto f * \check{\nu}$. If the representation 
(2.4.2) is normal it follows that the operator $T$ is self adjoint with respect to $d^{r} x=d^{r} r \otimes d k$ if and only if

$$
L(h, k)=L(k, h), \quad \check{\mu}_{h, k}=\mu_{k, h} .
$$

Let now $\tilde{d} x=\tilde{d} r \otimes d k$ be some measure on $X$ defined as in Section 2.2 , it is then clear from (2.4.1) that the $L^{p}(X ; \tilde{d} x) \rightarrow L^{q}(X ; \tilde{d} x)$ norm can be controlled by the $* \mu_{k_{1}, k_{2}} * \cdots \mu_{k_{n-1}, k_{n}}: L^{p}(R ; \tilde{d} r) \rightarrow L^{q}(R ; \tilde{d} r)$ convolution norm, i.e.

$$
\left\|T_{1} \circ \cdots \circ T_{n}\right\|_{p \rightarrow q} \leq\left\|L_{1} \circ \cdots \circ L_{n}\right\|_{p \rightarrow q} \sup _{k_{1} \cdots k_{n}}\left\|* \mu_{k_{1}, k_{2}} * \cdots * \mu_{k_{n-1}, k_{n}}\right\|_{p \rightarrow q}
$$

where $L_{1}, \ldots, L_{n} ; L_{1} \circ \cdots \circ L_{n}$ are the corresponding operators on $K$ as in Section 2.2. If the representations (2.4.2) are all normal and if $\tilde{d} r=d^{r} r, \tilde{d} x=d^{r} x$ this means that

$$
\begin{aligned}
\left\|T_{1} \circ \cdots \circ T_{n}\right\|_{p \rightarrow p} & \leq\left\|L_{1} \circ \cdots \circ L_{n}\right\|_{p \rightarrow p}, \\
\left\|T^{n}\right\|_{p \rightarrow p} & \leq\left\|L^{n}\right\|_{p \rightarrow p}, \quad 1 \leq p \leq+\infty,
\end{aligned}
$$

we also have the following basic

Lemma. If $R$ is amenable, the above inequality is actually an equality i.e.

$$
\left\|T_{1} \circ \cdots \circ T_{n}\right\|_{p \rightarrow p}=\left\|L_{1} \circ \cdots \circ L_{n}\right\|_{p \rightarrow p}, \quad 1 \leq p \leq+\infty .
$$

Proof. Since "everything is positive" it suffices to show that there exist $0 \leq f_{m}, g_{m} \in C_{0}^{\infty}(R)(m \geq 1)$ such that

$$
\left\|f_{m}\right\|_{p} \leq 1, \quad\left\|g_{m}\right\|_{q} \leq 1, \quad m \geq 1, \quad \frac{1}{p}+\frac{1}{q}=1
$$

$$
\left\langle f_{m} * \mu_{k_{1}, k_{2}} * \cdots * \mu_{k_{n-1}, k_{n}}, g_{m}\right\rangle_{L^{2}\left(R ; d^{r} r\right)} \underset{m \rightarrow \infty}{\longrightarrow} 1
$$

and that the limit in (2.4.5) uniform when $k_{j} \in C \subset \subset K(j=1, \ldots, n)$ and $C$ is a compact subset. In fact, to avoid unnecessary complications in this proof we shall make an additional hypothesis that will always be verified for us: We shall assume that for all $\varepsilon>0$ exists $C \subset R$ such 
that $\mu_{h, k}(R \sim C)<\varepsilon(h, k \in K)$. Otherwise the next few lignes have to be "handled with care"!

If we denote by $\pi=\mu_{k_{1}, k_{2}}^{(1)} * \cdots * \mu_{k_{n-1}, k_{n}}^{(n)}$ we see that

$$
\begin{aligned}
\langle f * \pi, g\rangle_{L^{2}\left(R ; d^{r} r\right)} & =\int g(x) d^{r} x \int f\left(x y^{-1}\right) d \pi(y) \\
& =\int\left(\int f\left(x y^{-1}\right) g(x) d^{r} x\right) d \pi(y) \\
& =\int\left(\int \tilde{f}\left(y x^{-1}\right) g(x) d^{r} x\right) d \pi(y)=\left\langle\tilde{f}^{*} g, \pi\right\rangle,
\end{aligned}
$$

where $\tilde{f}(x)=f\left(x^{-1}\right)$ and where the defintion of the right convolution $\stackrel{*}{r}$ of two functions is "given" by the last equality. (2.4.4), (2.4.5) will therefore follow if we can choose $f_{m}, g_{m} \in C_{0}^{\infty}$ that satisfy (2.4.4) and such that

$$
\tilde{f}_{m} \stackrel{*}{r} g_{m} \underset{m \rightarrow \infty}{\longrightarrow} 1 \quad \text { uniformly on compacta }
$$

The well informed reader recognizes here one of the many consequences and definitions of the amenability ( $c f$. [16] where it is proved in its dual form $\|f\|_{p},\|g\|_{q} \leq 1$ where the \|\| are taken with respect to the left measure $d r$, and $f_{m} * \tilde{g}_{m} \rightarrow 1$. Observe also that one way to avoid the uniformity hypothesis on the measures $\mu_{h, k}$ is to impose some kind of monotonicity on the limit (2.4.6)).

A very important conclusion can be drawn from the above consideration. Let us start from the assumption that for some $0 \leq \theta(n) \underset{n \rightarrow \infty}{\rightarrow} 0$ and for every fixed $\varphi, \psi \in C_{0}^{\infty}(R)$ we have

$$
\sup _{k_{1}, \ldots, k_{n}}\left\langle\varphi * \mu_{k_{1}, k_{2}}^{(1)} * \cdots * \mu_{k_{n-1}, k_{n}}^{(n)}, \psi\right\rangle_{L^{2}\left(R ; d^{r} r\right)}=O(\theta(n)) .
$$

It then follows that for fixed $F=\varphi_{1} \otimes \varphi_{2}, \Psi=\psi_{1} \otimes \psi_{2}, \varphi_{1}, \psi_{1} \in C_{0}^{\infty}(R)$, $\varphi_{2}, \psi_{2} \in C_{0}^{\infty}(K)$ we have

$$
\left\langle T^{n} F, \Psi\right\rangle=O\left(\theta(n)\left\|L^{n}\right\|_{2 \rightarrow 2}\right)
$$

where for simplicity we assume that $T_{1}=T_{2}=\cdots=T$. It follows that if $R$ is amenable we have the local estimate

$$
\left\langle T^{n} F, \Psi\right\rangle=O\left(\theta(n)\left\|T^{n}\right\|_{2 \rightarrow 2}\right) .
$$


One final remark is in order. Let $\alpha(r)>0$ be an arbitrary continuous positive function and $T_{\alpha}=\alpha^{1 / 2} T \alpha^{-1 / 2}$ the corresponding conjugated operator. It is clear that $T_{\alpha}^{n}=\alpha^{1 / 2} T^{n} \alpha^{-1 / 2}$ and that the $2 \rightarrow 2$ norm of $T_{\alpha}$ with respect to $\tilde{d} x=\tilde{d} r \otimes d k$ is the same as the $2 \rightarrow 2$ norm of $T$ with respect to $\alpha^{-1} \tilde{d} x=\left(\alpha^{-1} \tilde{d} r\right) \otimes d k$. The local estimate is on the other hand invariant by that conjugation since the $\alpha$ is absorbed in the compactly supported $F$ and $\Psi$. In other words for arbitrary $\alpha$ as above we have

$$
\left\langle T^{n} F, \Psi\right\rangle=O\left(\theta(n)\left\|T^{n}\right\|_{L^{2}\left(\alpha d^{r} x\right) \rightarrow L^{2}\left(\alpha d^{r} x\right)}\right)
$$

and in particular, with $\alpha=m_{R}=$ the modular function of $R$, we have

$$
\left\langle T^{n} F, \Psi\right\rangle=O\left(\theta(n)\left\|T^{n}\right\|_{L^{2}(d x) \rightarrow L^{2}(d x)}\right) .
$$

The proof of the upper estimate of our main theorem hindges on this observation.

Let us now suppose that the density $L(h, k)$ of the operator $L$ is continuous and strictly positive and that the operator $L: L^{2}(K) \rightarrow$ $L^{2}(K)$ is compact. In the above estimate we can then replace $\|T\|_{2 \rightarrow 2}$ by $\|T\|_{\mathrm{sp}}$ the spectral radius of $T$ (since $\|A\|_{\mathrm{sp}}=\lim \left\|A^{n}\right\|^{1 / n}$ we clearly have $\left.\|T\|_{\mathrm{sp}}=\|L\|_{\mathrm{sp}}\right)$.

The reason why we can do this is because the operator $L$ admits then $0<\varphi_{0} \in L^{2}(K)$ a positive eigenfunction (cf. [31]) and in the previous argument we can set $\varphi_{2}=\psi_{2}=\varphi_{0}$.

Indeed assume for simplicity that $K$ is compact then any eigenfunction of $L$ is continuous and if $\varphi \in C(K)$ is such an eigenfunction with maximal (in modulus) eigenvalue then

$$
L|\varphi| \geq|L \varphi| ; \quad L|\varphi| \ngtr(1+\varepsilon)|L \varphi| \quad \text { for all } \varepsilon>0,
$$

i.e. the inequality $L|\varphi|>(1+\varepsilon)|L \varphi|$ does not hold for any $\varepsilon>0$ (indeed if we assume, as we may, that the eigenvalue in question has modulus 1 , such an inequality would give $\left\|L^{n}\right\| \geq(1+\varepsilon)^{n}$ which contradicts the fact that $\|L\|_{\mathrm{sp}}=1$ ).

Therefore there exists $k_{0} \in K$ such that $L|\varphi|\left(k_{0}\right)=\left|L \varphi\left(k_{0}\right)\right|$. But this (because $L>0$ ) implies that $\varphi=e^{i \theta}|\varphi|$ (for some fixed $\theta \in \mathbb{R}$ ). We have therefore $L|\varphi|=\|L\|_{\mathrm{sp}}|\varphi|$ and from this it follows that $\varphi_{0}=$ $|\varphi|>0$. 


\subsection{Semigroup of operators.}

In this section I shall examine

$$
T_{t}=L_{t}(h, d k) \otimes\left\{* \mu_{h, k}^{(t)}\right\}, \quad t>0,
$$

a semigroup of positive $R$-left invariant operators on $X$, i.e. we assume that $T_{t} \circ T_{s}=T_{t+s}$. We shall assume that the representation (2.5.1) is normal, by (2.2.4) it then follows that $L_{t} \circ L_{s}=L_{t+s}$ (cf. Section 2.2) is also a semigroup and that if $T_{t}$ is symmetric with respect to $d^{r} r \otimes d k$ then $T_{t} \gg 0$, i.e. is a positive Hilbert space operator with respect to that measure. Clearly also $T_{t}$ is (sub)markovian if and only if $L_{t}$ is (sub)markovian.

ExAmple. $T_{t}=e^{-t A}$ where $A$ is a $R$-left invariant differential operator on $X$. To write down $A$ we can fix once and for all left invariant fields on $R, Y_{1}, Y_{2}, \ldots$ and local coordinates $\left(k_{1}, k_{2}, \ldots\right)$ on $K$. It follows that $Y_{j}$ and $\partial / \partial k_{i}$ commute and that we can write

$$
A=\sum a_{i j} Z_{i} Z_{j}+\sum a_{i} Z_{i}+a
$$

where each $Z_{i}$ is either one of the $Y_{j}$ 's or one of the $\partial / \partial k_{j}$ 's and furthermore each coefficient $a_{i j}, a_{i}, a$ is independent of $r \in R$ (but may depend on $k \in K)$. The "projected" operator $B$ on $K$ is then obtained by retaining only the terms of (2.5.2) for which no $Y_{j}$ field appears and we have $L_{t}=e^{-t B}$. Observe that in the group case $T_{t}$ is a " $K$-biinvariant" semigroup if $A$ is a $K$-bi-invariant and that this implies that $B$ is $K$-right invariant on $K$. If we are in a group case $X=G$ we can, for instance, take

$$
A=\sum b_{i j} X_{i} X_{j}+X_{0}+b
$$

where the $b$ 's are constant with $\left(b_{i j}\right) \gg 0$ and $X_{1}, X_{2}, \ldots$ are left invariant vector fields on $G$. Such an operator can clearly be rewritten

$$
\Delta=\sum_{i=1}^{n} X_{i}^{2}+X_{0}+b
$$

as in Section 0 (for a different, of course, choice of invariant fields $\left.X_{0}, X_{1}, \ldots\right)$. When $b=0, \Delta$ is a markovian generator on $G$ and is 
formally selfadjoint with respect to $d^{r} g$ if $X_{0}=0$. But even if $X_{0}=0$, $\Delta$ is not in general formally selfadjoint with respect to $d^{r} r \otimes d k$ and therefore the corresponding operators $e^{-t B}$ are markovian but not symmetric. There is another important property that $B$ inherits from $A$. First of all if $A$ is elliptic (i.e. if the matrix $\left(a_{i j}\right)$ is positive definite) the operator $B$ is also elliptic. Let us assume more generally that the fields $X_{1}, \ldots, X_{n}$ in (2.5.4) generate the Lie algebra of $G$. The projected operator $B$ can then be written

$$
B=\sum_{i=1}^{n} \tilde{X}_{j}^{2}+\tilde{X}_{0}+\tilde{b}
$$

where $\tilde{X}_{j}$ is the corresponding projected field on $K$. Of course, even if we are in the group case, $\tilde{X}_{j}$ need not be in general a $K$-invariant field in any sense whatsoever, but it is certainly true that $B$ is on $K$ a Hörmander operator in the sense that at every point $k \in K$ the fields $\tilde{X}_{1}, \ldots, \tilde{X}_{n}$ span together with all their successive brackets the tangent space.

Let us now go back to the general semigroup and let us assume that $T_{t}$ is symmetric with respect to $d^{r} x$ and that therefore $L_{t}$ is symmetric with respect to $d k$ (both $T_{t}$ and $L_{t}$ are therefore positive operators in the Hilbert space sense). I shall further make the following assumption: $A$ : (respectively $\left.A^{\prime}\right)$ : there exists $\varphi_{0}>0, \lambda_{0}>0$ such that

$$
L_{t} \varphi_{0}=e^{-\lambda_{0} t} \varphi_{0} \quad\left(\text { respectively } L_{t} \varphi_{0} \leq e^{-\lambda_{0} t} \varphi_{0}\right) \text {. }
$$

We shall presently elaborate on that condition but first we shall draw the consequences of $A$ and $A^{\prime}$. Under the above conditions we shall consider the semigroups

$$
\hat{T}_{t}=e^{\lambda_{0} t} \varphi_{0}^{-1} T_{t} \varphi_{0}, \quad \hat{L}_{t}=e^{\lambda_{0} t} \varphi_{0}^{-1} L_{t} \varphi_{0}
$$

where in (2.5.6) $\varphi_{0}$ is identified with an $R$-left invariant function on $X$ which satisfies $T_{t} \varphi_{0}=e^{-\lambda_{0} t} \varphi_{0}$ (respectively $T_{t} \varphi_{0} \leq e^{-\lambda_{0} t} \varphi_{0}$ ) on $X$. The semigroups (2.5.6) are therefore markovian (respectively submarkovian) $\hat{L}_{t}$ is symmetric with respect to the measure $\hat{d k}=\varphi_{0}^{2} d k$ and $\hat{T}_{t}$ is symmetric with respect to the measure $d^{r} r \otimes \hat{d} k$.

Let us now go back to the assumption $A$ (and $A^{\prime}$ ) and give natural examples under which it is verified. Let us first suppose that we are in the group case that $K$ is compact and that $T_{t}=e^{-t \Delta}$ with $\Delta$ as in 
(2.5.4). To simplify matters let us also assume that $L_{t}$ is symmetric with respect to $d k$ ( $d k$ here is some smooth non vanishing measure that need not be the Haar measure of $K$ ). The Hörmander condition on (2.5.4) implies that the operators $L_{t}$ are in the trace-class on $L^{2}(K ; d k)$. This is because the kernel $L_{t}(h, k)$ is $C^{\infty}$ and thus Hilbert-Schmidt (and $\left.L_{t / 2} \circ L_{t / 2}=L_{t}\right)$. We have therefore

$$
L_{t}=\sum_{j=0}^{\infty} e^{-\lambda_{j} t} \varphi_{j}(h) \varphi_{j}(k)
$$

where $\lambda_{0} \leq \lambda_{1} \leq \cdots$ with $\sum_{j=0}^{\infty} e^{-\lambda_{j} t}<\infty(t>0)$, and $\varphi_{j} \in C_{\mathbb{R}}^{\infty}(K)$, $\left\|\varphi_{j}\right\|_{2}=1, j=0,1,2, \ldots$.

By the positivity of the operators involved we have $L_{t}\left|\varphi_{0}\right| \geq\left|L_{t} \varphi_{0}\right|$ $=e^{-\lambda_{0} t}\left|\varphi_{0}\right|$. Also, since $\left\|L_{t}\right\|_{2 \rightarrow 2}=e^{-\lambda_{0} t}$, we have $\left\|L_{t}\left|\varphi_{0}\right|\right\|_{2}$ $\leq e^{-\lambda_{0} t}|| \varphi_{0} \|_{2}$ and therefore $L_{t}\left|\varphi_{0}\right|=e^{-\lambda_{0} t}\left|\varphi_{0}\right|$. It follows that we can renumber the eigenfunctions $\varphi_{0}, \varphi_{1}, \ldots$ in such a way that $0 \leq$ $\varphi_{0} \in C^{\infty}(K)$.

The next step is to show that $\varphi_{0}$ never vanishes $\varphi_{0}>0(k \in K)$ and that therefore the condition $(A)$ is verified. This of course is an immediate consequence of the eigenvalue property

$$
L_{t} \varphi_{0}(h)=\int L_{t}(h, k) \varphi_{0}(k) d k=e^{-\lambda_{0} t} \varphi_{0}(h)
$$

and of the more general fact that for any non identically zero $0 \leq \varphi \in$ $C^{\infty}(K)$ we have

$$
L_{t} \varphi(h)>0, \quad h \in K .
$$

To see this we observe that $L_{t}=e^{-t(B-\alpha)} e^{-t \alpha}$ for any $\alpha>0$ and $B$ as in (2.5.5). $B-\alpha$, on the other hand, for $\alpha \geq 0$ large, generates a "hypoelliptic" diffusion. This means that the kernel $e^{t \alpha} L_{t}(h, k)$ never vanishes for $t>0$ (cf. [4]), (2.5.7) follows.

The above situation can be generalized as follows. We shall drop the assumption that $K$ is compact but assume that there exists $\Gamma$ some discrete group that acts discontinuously on $K$ and in such a way that $K / \Gamma$ is compact. We shall also assume that $T_{t}$ the semigroup (2.5.1) is stable by the natural $\Gamma$-action and induces thus a corresponding $R$-left invariant semigroup on $X / \Gamma=R \times K / \Gamma$. If we assume that the corresponding semigroup $L_{t}$ on $K / \Gamma$ has all the above properties (so that the existence of $\varphi_{0}$ on $K / \Gamma$ with the required properties is guaranteed) then 
we can define the analogous $\varphi_{0}$ on $K$ by taking on $K$ the corresponding $\Gamma$-automorphic function. We see in particular that the assumption $A$ is verified in our group case vi) of Section 2.1.

\subsection{The symmetric Laplacian in the group case.}

Let us suppose that we are in the group case $G=X=R \times K$ as in Section $2.1 \mathrm{vi}$ ) and that $\Delta=-\sum X_{j}^{2}, T_{t}=e^{-t \Delta}$ is as in Section 0.1. The modified semigroup $\tilde{T}_{t}=m_{G}^{1 / 2} T_{t} m_{G}^{-1 / 2}$ is then symmetric with respect to $d^{\ell} g=d^{\ell} r \otimes d k$ and therefore

$$
\check{T}_{t}=m_{R}^{-1 / 2} \tilde{T}_{t} m_{R}^{1 / 2}=\left(m_{G} / m_{R}\right)^{1 / 2} e^{-t \Delta}\left(m_{G} / m_{R}\right)^{-1 / 2}
$$

is symmetric on $X$ with respect to $d^{r} x=d^{r} r \otimes d k$ as was needed for the considerations of Section 2.5 to go through. Observe that when $K=\{e\}, m_{R} \equiv m_{G}$ and $\check{T}_{t}=T_{t}$.

In this section I shall make a number of explicit computations related to the above semigroup. Let $G$ be a simply connected Lie group and let $G=Q<S, S=N A K, R=Q N A, Z \subset K$ have the same meaning as in Section 2.1 so as to have the identification $G=R \times K$. It is clear that $m_{G}(k) \equiv 1(k \in K)$ (and more generally $\left.m_{G}\right|_{S}=1$ ) so it suffices to analyse $m_{R}$ and $\left.m_{G}\right|_{R}$. Since $Q$ is a normal subgroup of $G$ we have $\left.m_{G}\right|_{Q}=\left.m_{R}\right|_{Q}=m_{Q}$ so that $m_{G} /\left.m_{R}\right|_{Q} \equiv 1$. Since $S$ is semisimple and $\left.m_{G}\right|_{S} \equiv 1$ it follows that

$$
\left(m_{G} / m_{R}\right)^{1 / 2}(x)=m_{R}^{-1 / 2}(x), \quad x \in A N .
$$

Now since all the automorphisms induced on $Q$ by inner automorphisms by elements of $S$ are unimodular ( $S$ being semisimple) we have ( $c f$. [25])

$$
m_{R}(x)=m_{A N}(x), \quad x \in A N
$$

So with the obvious abuse of notation we have

$$
\left(m_{G} / m_{R}\right)^{1 / 2}=m_{A N}^{-1 / 2},
$$

where $G=(Q \wedge A N) K$. For the semisimple group $S$ the quantity $m_{A N}$ is a very familiar creature $m_{A N}=e^{2 \rho}$ where $\rho=\left(\sum \rho_{j}\right) / 2$ is the $1 / 2$-sum of the roots $(c f$. [9]). In particular it only depends on the 
$A$-coordinate. The "moral" is that the conjugating factor in (2.6.1) is an "old friend".

To obtain the symmetric markovian semigroup $\hat{T}_{t}$ on $G$ then we define as in Section 2.5 the $Z$-automorphic function on $K, \varphi_{0}$ and $\lambda_{0}$ the corresponding eigenvalue which is then given by $e^{-\lambda_{0} t}=\left\|\check{T}_{t}\right\|_{2 \rightarrow 2}$ on $L^{2}\left(X ; d^{r} x\right)$ (cf. Section 2.4). But clearly also $e^{-\lambda_{0} t}=\left\|\tilde{T}_{t}\right\|_{2 \rightarrow 2}$ on $L^{2}(G ; d g)$ and $\lambda_{0}=\lambda$ is just the spectral gap (cf. Section 0.1 ) of $\Delta$. The semigroup

$$
\hat{T}_{t}=e^{\lambda t} \varphi_{0}^{-1} \check{T}_{t} \varphi_{0}=e^{\lambda t} \varphi_{0}^{-1}\left(m_{G} / m_{R}\right)^{1 / 2} e^{-t \Delta}\left(m_{G} / m_{R}\right)^{-1 / 2} \varphi_{0}
$$

is thus markovian and symmetric with respect to $d^{r} r \otimes \varphi_{0}^{2} d k=d^{r} r \otimes \hat{d} k$.

Let now $\hat{\phi}_{t}\left(x_{1}, x_{2}\right)$ be the kernel of the semigroup $\hat{T}_{t}$ with respect to $d r \otimes \hat{d} k$ we shall show then that we have

$$
\begin{aligned}
\hat{\phi}_{2 t}(e, e) & \approx e^{2 \lambda t} \int_{G} \phi_{t}(g) \phi_{t}\left(g^{-1}\right) d g \\
& =e^{2 \lambda t} \int \phi_{t}\left(k^{-1} r^{-1}\right) \phi_{t}(r k) d r d k
\end{aligned}
$$

where $\phi_{t}\left(y^{-1} x\right)$ is the convolution kernel of $T_{t}=e^{-t \Delta}$ with respect to $d g$. Indeed quite generally if $k_{t}\left(\omega_{1}, \omega_{2}\right)$ is the kernel with respect to $d \omega$ of a general semigroup $K_{t}$ on $L^{2}(\Omega ; d \omega)$ where $(\Omega ; d \omega)$ is some measure space then $k_{t}^{(\alpha)}\left(\omega_{1}, \omega_{2}\right)$ the kernel of the conjugated semigroup $\alpha K_{t} \alpha^{-1}$ with respect to $d \omega$ (where $\alpha(\omega) \neq 0$ is some non zero function) is $k_{t}^{(\alpha)}\left(\omega_{1}, \omega_{2}\right)=k_{t}\left(\omega_{1}, \omega_{2}\right)\left(\alpha\left(\omega_{1}\right) / \alpha\left(\omega_{2}\right)\right)$. This in particular implies

$$
k_{t}^{(\alpha)}\left(\omega_{1}, \omega_{2}\right) k_{t}^{(\alpha)}\left(\omega_{2}, \omega_{1}\right)=k_{t}\left(\omega_{1}, \omega_{2}\right) k_{t}\left(\omega_{2}, \omega_{1}\right), \quad t>0 .
$$

Similarly the kernel of $K_{t}$ with respect to a new measure $\beta(\omega) d \omega$ is

$$
k_{t}^{\langle\beta\rangle}\left(\omega_{1}, \omega_{2}\right)=\frac{1}{\beta\left(\omega_{2}\right)} k_{t}\left(\omega_{1}, \omega_{2}\right)
$$

If we apply these observations in our context where $\beta=\varphi_{0}^{2}$ is bounded from above and below we deduce that

$$
C^{-1} \phi_{t}(x) \phi_{t}\left(x^{-1}\right) \leq e^{-2 \lambda t} \hat{\phi}_{t}(e, x) \hat{\phi}_{t}(x, e) \leq C \phi_{t}(x) \phi_{t}\left(x^{-1}\right) .
$$

(2.6.3) follows then from

$$
\hat{\phi}_{2 t}(e, e)=\int_{X} \hat{\phi}_{t}(e, x) \hat{\phi}_{t}(x, e) d r \hat{d k}
$$


Observe now that the symmetry of $\hat{T}_{t}$ with respect to $d^{r} r \otimes \hat{d} k$ implies that

$$
\hat{\phi}_{t}\left(x, x_{1}\right) m_{R}^{-1}\left(r_{1}\right)=\hat{\phi}_{t}\left(x_{1}, x\right) m_{R}^{-1}(r), \quad x=(r, k), x_{1}=\left(r_{1}, k_{1}\right),
$$

and therefore also

$$
P(t)=\int \hat{\phi}_{t}(e, x) \hat{\phi}_{t}(x, e) d^{r} r \hat{d} k=\int \hat{\phi}_{t}^{2}(e, x) d r d \hat{k} .
$$

From (2.6.4) it follows also that

$$
C^{-1} P(t) \leq e^{2 \lambda t} \int \phi_{t}(r k) \phi_{t}\left(k^{-1} r^{-1}\right) d^{r} r \hat{d} k \leq C P(t) .
$$

We shall now show that for large $t \gg 1$ both $\hat{\phi}_{2 t}(e, e)$ and $P(t)$ are "comparable" with the quantity

$$
Q(t)=e^{2 \lambda t} \int \phi_{t}(r) \phi_{t}\left(r^{-1}\right) d r=e^{2 \lambda t} \int \phi_{t}(r) \phi_{t}\left(r^{-1}\right) d^{r} r
$$

in the sense that

Lemma. If $K$ is compact, there exists $C>0$ such that

$$
\begin{gathered}
C^{-1} Q(t-1) \leq \hat{\phi}_{2 t}(e, e) \leq C Q(t+1), \quad t \geq 10, \\
C^{-1} Q(t-1) \leq P(t) \leq C Q(t+1), \quad t \geq 10 .
\end{gathered}
$$

It follows in particular that

$$
\begin{aligned}
C^{-1} \int \hat{\phi}_{t-2}^{2}(e, x) d r \hat{d} k & \leq \hat{\phi}_{2 t}(e, e) \\
& \leq C \int \hat{\phi}_{t+2}^{2}(e, x) d r d \hat{k}, \quad t \geq 20
\end{aligned}
$$

Proof. If $K$ is compact by the standard local Harnack estimate ( $c f$. [1], [4]) it follows that

$C^{-1} \phi_{t-1 / 2}\left(g k_{2}\right) \leq \phi_{t}(g) \leq C \phi_{t+1 / 2}\left(g k_{1}\right), \quad t>1, g \in G, k_{1}, k_{2} \in K$, where $C>0$ is independent of $t, g$ and $k_{1}, k_{2}$. 
Combining these with the fact that

$$
\phi_{t}\left(g^{-1}\right)=\phi_{t}(g) m_{G}(g), \quad g \in G .
$$

We deduce that

$$
C^{-1} \phi_{t-1}\left(k_{3} g k_{4}\right) \leq \phi_{t}(g) \leq C \phi_{t+1}\left(k_{1} g k_{2}\right)
$$

for $t>10, g \in G, k_{i} \in K, 1 \leq i \leq 4$. If $K$ is compact the integrals in both (2.6.3) and (2.6.6) are comparable (in the above sense) with $\int \phi_{t}(r) \phi_{t}\left(r^{-1}\right) d r$ and our lemma follows. If $K$ is not compact we shall choose $K_{0}$ some relatively compact fundamental domain of the covering map $K \rightarrow K / Z$ so that

$$
K=\bigcup_{z \in Z} z K_{0}, \quad z_{1} K_{0} \cap z_{2} K_{0}=\varnothing, \quad z_{1}, z_{2} \in Z, z_{1} \neq z_{2}
$$

What replaces $(2.6 .9)$ is then the estimate

$$
C^{-1} \phi_{t-1}\left(z k_{3} g k_{1}\right) \leq \phi_{t}(z g) \leq C \phi_{t+1}\left(z k_{1} g k_{2}\right), \quad t>20,
$$

where $z \in Z$ is central in $G$. The above argument therefore works provided that in $(2.6 .7)$ we now set

$$
\begin{aligned}
Q(t) & =e^{2 \lambda t} \int_{R} \sum_{z \in Z} \phi_{t}(z r) \phi_{t}\left(z^{-1} r^{-1}\right) d r \\
& =e^{2 \lambda t} \int_{R} \sum_{z \in Z} \phi_{t}(z r) \phi_{t}\left(z^{-1} r^{-1}\right) d^{r} r .
\end{aligned}
$$

We conclude therefore that (2.6.8) is valid in full generality.

In all the above considerations we used the measure $\hat{d} k=\varphi_{0}^{2} d k$ on $K$ and the corresponding measure $\hat{d} k=d^{r} r \otimes \hat{d} k$ on $X$ with respect to which the semigroup $\hat{T}_{t}$ in (2.6.2) is symmetric. It turns out that if we invoque a result of J. Moser [17] we can in fact replace $\hat{d} k$ by the Haar measure $d k$. J. Moser's result says that when $K$ is compact and orientable there exists a diffeomorphism $\alpha: K \rightarrow K$ that takes the measure $\hat{d} k$ to $d k$. If we use this diffeomorphism and conjugate $\hat{T}_{t}$ with $\alpha=$ identity $\otimes \alpha$ on $X$ i.e. $(f \circ \alpha) \mapsto\left(\hat{T}_{t} f\right) \circ \alpha$ (for all $\left.f \in C_{0}^{\infty}(X)\right)$ we obtain a new semigroup that I shall still denote by $\hat{T}_{t}$ which is markovian and symmetric with respect to $d^{r} r \otimes d k$. The same thing of 
course holds in the general case (i.e. $K$ is not compact) provided that we can lift the diffeomorphism from the compact manifold $K / Z$ on $K$. This diffeomorphism lifts automatically when $K$ is simply connected.

We shall finish this section with a probabilistic interpretation of the lower estimates in the Theorem A. Towards that we shall consider $\Omega=\{x(t) \in X ; t>0\}$ the path space of the diffusion on $X$ generated by the semigroup $\hat{T}_{t}$. In other words

$$
\begin{gathered}
\hat{T}_{t} f(x)=\int \mathbb{P}_{x}[x(t) \in d y] f(y), \quad f \in C_{0}^{\infty}(X), \\
\mathbb{P}_{e}[x(t) \in A]=\int_{x=(r, k) \in A} \hat{\phi}_{t}(e, x) d r \otimes \hat{d k} .
\end{gathered}
$$

If we bare in mind that $c^{-1} d k \leq \hat{d} k \leq c d k$ and combine this with our main estimate (2.6.8) we see that

$$
\mathbb{P}[x(t) \in A] \leq C\left(\hat{\phi}_{t}(e, e)\right)^{1 / 2}(d x \text {-measure }(A))^{1 / 2} .
$$

The $d x=d r \otimes d k$ measure of $A \subset G=R \times K$ is of course the left Haar measure on $G$. If we use however the involution $*:(r, k) \rightarrow\left(r^{-1}, k\right)$ we see from the symmetry of $\hat{T}_{t}$ with respect to $d^{r} r \otimes \hat{d} k$ that if $A$ is of the form $A=B \times K(B \subset R)$ then

$$
\mathbb{P}[x(t) \in A]=\mathbb{P}\left[x(t) \in A^{*}\right]
$$

and since $*$ interchanges the two measures $d^{r} r \otimes \hat{d} k$ and $d r \otimes \hat{d} k$ we see finally that in (2.6.10), if we so wish, we can replace the $d x$-measure by any of the measures $d^{r} r \otimes d k, d^{r} r \otimes \hat{d} k, d^{\ell} r \otimes \hat{d} k$. The estimate (2.6.10) allows us to formulate the following criterion.

Criterion. Let us assume that for all $n=1,2, \ldots$ we can find a set $X_{n}=B_{n} \times K \subset X\left(B_{n} \subset R\right)$ such that

i) measure $\left(X_{n}\right) \leq C n^{C}, \quad n=1,2, \ldots$

ii) $\mathbb{P}\left[x(n) \in X_{n}\right] \geq C^{-1} n^{-C}, \quad n=1,2, \ldots$

where $C>0$ and "measure" stands for any of the above measures. Then there exists $C>0$ such that $\phi_{t}$, the convolution kernel of $e^{-t \Delta}$, satisfies

$$
\phi_{t}(e) \geq C^{-1} t^{-C} e^{-\lambda t}, \quad t \geq 1 .
$$


The standard local Harnack principle ( $c f$. [1]) has to be used of course here to fill in the gaps between the integer values $t=1,2, \ldots$.

We shall also need (for the lower estimate in Theorem $A_{1}$ ) a modified version of the above criterion: If $X_{n}$ is as above but instead of i) and ii) we can only assert that

i') measure $\left(X_{n}\right) \leq C e^{c n^{1 / 3}}$

ii') $\mathbb{P}\left[x(n) \in X_{n}\right] \geq c^{-1} e^{-c n^{1 / 3}}, \quad n=1,2, \ldots$

Then we can conclude instead that

$$
\phi_{t}(e) \geq C^{-1} e^{-c t^{1 / 3}} e^{-\lambda t} .
$$

\subsection{The projection of the infinitesimal generator.}

In this section I shall preserve all our previous notations and assume that $N \subset R$ is some closed normal subgroup. We can define then $\pi: X=R \times K \rightarrow X / N=R / N \times K$ the quotient spaces by the induced left action by $N$ and if $T=L \otimes\left\{* \mu_{h, k}\right\}$ is a positive left invariant on $X$ as in Section 2.1 the above projection induces $T_{X / N}=L \otimes\left\{* \check{\pi}(\mu)_{h, k}\right\}$ a positive left invariant operator on $X / N(\check{\pi}(\mu)$ denotes here the image of the measure $\mu$ by $\pi$ ).

It is clear then that if $T$ is self adjoint with respect to the measure $d^{r} r \otimes d k$ then $T_{X / N}$ is self adjoint with respect to $d_{R / N}^{r} r \otimes d k$ (We can use the criterion (2.4.3) to see this).

We shall now give an important example of the above situation. We shall assume that $R$ is a simply connected soluble Lie group and that $N$ is the nilradical so that $R / N \cong \mathbb{R}^{n}$. The right measure on $R / N$ is then the Lebesgue measure $d x$. We shall further assume that we are in the group case and that the left invariant operators considered are the $\hat{T}_{t}$ defined in Section 2.6 which will be self adjoint with respect to $d^{r} r \otimes d k$ where $d k$ is now assumed to be the Haar measure on $K$ ( $c f$. end of Section 2.6). We clearly have $\hat{T}_{t}=e^{-t \hat{A}}$ where $\hat{A}$ is a sum of squares (with drift) operator that satisfies the Hörmander condition. We shall project as explained above and obtain $\hat{T}_{t}=e^{-t D}$ a symmetric (with respect to $d x \otimes d k$ ) markovian semigroup on $X / N=\mathbb{R}^{n} \times K$ and we shall analyze more closely $D$ the generator that is a subelliptic differential operator. 
Let $x_{1}, \ldots, x_{n}$ be the standard coordinates on $\mathbb{R}^{n}$ and let $X_{1}, \ldots$, $X_{s}$ be a basis of right invariant fields on $K$. It is then clear by the $\mathbb{R}^{n}$-left invariance that

$$
-D=D_{R}+M+P+D_{K},
$$

where

$$
D_{R}=\sum_{i, j=1}^{n} a_{i j}(k) \frac{\partial^{2}}{\partial x_{i} \partial x_{j}}
$$

where $\left(a_{i j}(k)\right)$ is a symmetric non negative matrix, $k \in K$.

$$
M=2 \sum_{i=1}^{n} \sum_{\alpha=1}^{s} b_{\alpha, i}(k) X_{\alpha} \frac{\partial}{\partial x_{i}}, \quad P=\sum_{i=1}^{n} \delta_{i}(k) \frac{\partial}{\partial x_{i}}
$$

and where $D_{K}$ can be identified with the canonical "projected operator" on $K$. That operator is self adjoint subelliptic and can thus be written in the form

$$
D_{K}=\sum_{\alpha, \beta=1}^{s} X_{\alpha} \gamma_{\alpha, \beta}(k) X_{\beta}
$$

where $\left(\gamma_{\alpha, \beta}(k)\right)$ is a symmetric non negative matrix. The constant term is zero because $D$ is a markovian generator. What is also clear is that $D_{R}$ in (2.7.2) is uniformily elliptic on $\mathbb{R}^{n}$ i.e. that

$$
\left(a_{i j}(k)\right) \geq \varepsilon_{0} I
$$

for some $\varepsilon_{0}>0$ provided that the original operator $\Delta$ on $G$ and therefore $D$ on $\mathbb{R}^{n} \times K$ is actually elliptic.

The formal self adjointness of $D$ with respect to $d x \otimes d k$ implies that

$$
\delta_{i}(k)=\sum_{\alpha=1}^{s} X_{\alpha} b_{\alpha, i}(k), \quad i=1,2, \ldots, n,
$$

and therefore that

$$
\int_{K} \delta_{i}(k) d k=0
$$


This is equivalent to the "formal" statement $\left\langle D\left(x_{i} \otimes 1\right), 1\right\rangle=0$.

Of course $D$ is $K$-bi-invariant if and only if all its coefficients are constant. This is the only reason why we choose the fields $X_{1}, \ldots, X_{s}$ to be right invariant rather than left invariant. If we use the canonical projection $\mathbb{R}^{n} \times K \rightarrow \mathbb{R}^{n}$ and project $D$ we obtain then $D_{R}$ on $\mathbb{R}^{n}$. It follows in particular that then $D_{R}$ is elliptic as soon as $D$ is subelliptic.

Let us finally examine the convolution kernel. Let us go back to the original semigroup $e^{-t \Delta} f=f * \mu_{t}$ with $d \mu_{t}(g)=\phi_{t}(g) d^{r} g$ then the corresponding left invariant operator on $R \times K$ is (cf. (2.3.6))

$$
\begin{aligned}
T_{t} f(r k) & =\iint f\left(r r_{1}^{-1} k_{1}\right) \phi_{t}\left(k_{1}^{-1} r_{1} k\right) d^{r} r_{1} d k_{1}, \\
T_{t} & =e^{-t \Delta}=L(h, d k) \otimes\left\{* \mu_{h, k}\right\} .
\end{aligned}
$$

But then clearly with $\tilde{M}=\varphi_{0}(k)\left(m_{R}(r) / m_{G}(r)\right)^{1 / 2}=\varphi_{0} M$ we obtain

$$
\tilde{M}^{-1} T_{t} \tilde{M}=\varphi_{0}^{-1}(h) \varphi_{0}(k) L(h ; d k) \otimes\left\{* M^{-1} \mu_{h, k}\right\},
$$

which means that

$$
\begin{array}{r}
\hat{T}_{t} f(r k)=e^{\lambda t} \varphi_{0}^{-1}(k) \iint \varphi_{0}^{-1}(h) f\left(r r_{1}^{-1} h\right) \phi_{t}\left(h^{-1} r_{1} k\right) \\
\cdot m_{G}^{1 / 2}\left(r_{1}\right) m_{R}^{1 / 2}\left(r_{1}\right) d r_{1} \hat{d} h
\end{array}
$$

Observe also that, with our previous notations, when $N \subset R$ is the nilradical of $R$ and $R / N=\mathbb{R}^{n}$ if we project the operator (2.7.7) on $\mathbb{R}^{n} \times K$ we obtain

$$
T_{t} f(x, k)=\iint f\left(x-x_{1}, k_{1}\right)\left(\int_{N} \phi_{t}\left(k_{1}^{-1} n x_{1} k\right) d n\right) d x_{1} d k_{1} .
$$

\subsection{Left invariant Markov chains and the semidirect product decomposition.}

We shall consider here $\left\{x_{n} \in X: n=1,2, \ldots\right\}$ a left invariant Markov chain as in Section 2.2 and assume that $R=N \wedge H$ is a semidirect product with $N \subset R$ a normal subgroup as in Section 2.7. We can identify here $X / N=R / N \times K$ with $Y=H \times K$ and $X=$ $N \times Y$. Let us denote by $\pi: X \rightarrow Y$ the canonical projection and by $\mathcal{Y}=\left\{y_{n}=\pi\left(x_{n}\right) \in Y: n=1,2, \ldots\right\}$ the corresponding left invariant 
chain on $Y$. With the above identifications we set $x_{n}=\left(z_{n}, y_{n}\right)\left(z_{n} \in\right.$ $\left.N, y_{n} \in Y, n=1, \ldots\right)$. We shall examine closely the process

$$
\mathcal{Z}=\left\{z_{n} \in N: n=1,2, \ldots\right\} .
$$

The process $\mathcal{Z}$ is not in general markovian but if we condition on the paths $\left(y_{1}, y_{2}, \ldots\right)$ of $\mathcal{Y} \mathcal{Z}$ becomes a Markov chain. This is a very important fact for us and we shall analyse it here in detail.

To help the reader see what is happening, let us first look at the special case when $N=R, Y=K$. If we use a normal representation

$$
T_{j}=L_{j}(h ; d k) \otimes\left\{* \mu_{h, k}^{(j)}\right\}
$$

of the transition operator, we see that conditionally on $\left(k_{1}, k_{2}, \ldots\right)$ $\left(k_{j} \in K\right)$ being fixed, the process $\left\{z_{n} \in R: n \geq 1\right\}$ is the Markov chain on $R$ with transition operators

$$
f \longmapsto f * \mu_{k_{j-1}, k_{j}}^{(j)}, \quad j=2,3, \ldots
$$

It is this idea that we generalize when $R=N \wedge H$. The key fact here is that any probability measure $\mu$ on $R$ can be disintegrated

$$
\mu=\int_{H} \lambda_{x} d \nu(x), \quad \nu \in \mathbb{P}(H), \lambda_{x} \in \mathbb{P}(x N), x \in H
$$

For simplicity again let us assume that $K=\{e\}$ is the one point set (this is the basic case treated in [13] and it will help the reader at this point to consult that references). The transition operator are then

$$
T_{j}=* \mu^{(j)}
$$

for probability measures on $R$

$$
\mu^{(j)}=\int_{H} \lambda_{y}^{(j)} d \nu^{(j)}(y)
$$

The measures $\lambda_{y}^{(j)}$ can (for every fixed $y \in H$ ) be identified to $\lambda_{y}^{(j)} \in$ $\mathbb{P}(N)$ by $z y \leftrightarrow z(z \in N)$ and since now $x_{n}=z_{n} y_{n}$ we easily see that with a fixed $\left(y_{1}, y_{2}, \ldots\right)$ the process $\left\{z_{1}, z_{2}, \ldots\right\}$ is a Markov chain on $N$ with transition operators

$$
f \longmapsto f * \pi_{j}
$$


where $\pi_{1}=\lambda_{y_{1}}$ and $\pi_{j}=\left(\lambda_{y_{j}}^{(j)}\right)^{y_{1} \cdots y_{j-1}}(j \geq 2)$ with the notation $\lambda^{x}$ $(\lambda \in \mathbb{P}(R) x \in R)$ for the image of $\lambda$ by the inner automorphism $g \mapsto$ $x g x^{-1}$. An alternative way of viewing the above situation is to observe that if we consider arbitrary measures $\lambda_{j} \in M(N)(j=1, \ldots, n)$ and place then on the cosets $N y_{j}$ by the identification $z \leftrightarrow z y_{j}$ then the convolution (in $R$ ) of these measures (that are placed on the cosets) lies in the coset $y_{1} y_{2} \cdots y_{n}$ and corresponds to the measure $\lambda_{1} * \lambda_{2}^{y_{1}} *$ $\lambda_{3}^{y_{1} y_{2}} * \cdots * \lambda_{n}^{y_{1} \cdots y_{n-1}}$ where now the convolution is taken in $N$.

The above two special cases $(K=\{e\}$ and $N=R)$ can now be put in the general context: we identify $X=R \times K=N \times Y=N \times H \times K$ so that $x_{n}=\left(r_{n}, k_{n}\right)=\left(z_{n}, h_{n}, k_{n}\right), y_{n}=\left(h_{n}, k_{n}\right) \in Y$ and with fixed $\left(y_{1}, y_{2}, \ldots\right)$ we disintegrate

$$
\mu_{k_{j-1}, k_{j}}=\int_{H} \lambda_{h}^{(j)} d \nu^{(j)}(h)
$$

$\left(z_{1}, z_{2}, \ldots\right)$ is then a Markov chain on $N$ with transition operators

$$
f \longmapsto f *\left(\lambda_{h_{j}}^{(j)}\right)^{h_{1} \cdots h_{j-1}}, \quad j=2, \ldots
$$

\subsection{Bi-invariant operators revisited.}

Nothing in this section is very new but I felt that it was appropiate to close this chapter by making the connection with know and standard ideas related to $K$-bi-invariant operators on semisimple groups.

Let $G$ be some Lie group that can be written $G=R \cdot K, R \cap K=$ $\{e\}$ for two closed subgroups with $Z \subset K$ as in Section 2.1 so that $\left.m_{G}\right|_{K} \equiv 1$. I shall consider on $G$ a differential operator $\Delta$ without constant term (i.e. $\Delta 1 \equiv 0$ ) that is $G$-left invariant $K$-right invariant and is in particular formally self adjoint and positive with respect to right measure $d^{r} g$. What we want is "somehow" to identify $\Delta$ with an operator on $R$. To do this we first conjugate $\Delta$ to $\tilde{\Delta}=m_{G}^{1 / 2} \Delta m_{G}^{-1 / 2}$ to make it formally self adjoint with respect to $d g=d^{\ell} g$. This of course creates a constant term $\tilde{\Delta} 1=C$ which in general is not zero. Let us consider $D=\tilde{\Delta}-C$ which is now a $G$-left, $K$-right invariant operator without constant term that is formally self invariant with respect to $d g$. From now onwards we shall consider operators $D$ that have the above properties. When $G=N A K$ is semisimple $D$, the classical $K$ bi-invariant Laplacian, has the above properties. 
Quite generally an operator on $G$ that has the above properties can be identified with a $G$-invariant operator on the homogeneous space $G / K=\{g K ; g \in G\}$ (When $G=N A K$ is semisimple $G / K$ is the symmetric space and the most important example of the above situation is that of the Laplace-Beltrami operator on $G / K)$. The homogeneous space $G / K$ can be identified with $R$, we obtain thus an identification of $D$ with an operator $D_{G / K}$ on $G / K$ and $D_{R}$ on $R$. $D_{R}$ is clearly $R$ left invariant has no constant term and since the $G$-invariant measure on $G / K$ (which always exists since $\left.m_{G}\right|_{K} \equiv 1 \equiv m_{K}$ ) can be identified with the left Haar measure of $R, D_{R}$ is formally self adjoint with respect to $d r=d^{\ell} r$ (indeed $D_{G / K}$ is clearly formally self adjoint with respect to the invariant measure on $G / K)$. It follows therefore that

$$
D_{R}=m_{R}^{1 / 2}\left(-\sum X_{j}^{2}+C_{R}\right) m_{R}^{-1 / 2},
$$

where $X_{1}, \ldots, X_{n}$ are left invariant fields on $R$. The only issue here is to determine the constant $C_{R}$. To do this let $\lambda_{D}$ be the spectral gap of operator $D_{G / K}$ on $G / K$. The operator $D_{R}-\lambda_{D}$ has then zero spectral gap on $L^{2}(R ; d r)$ and therefore $\tilde{D}_{R}=-\sum X_{j}^{2}+C_{R}-\lambda_{D}$ has zero spectral gap on $L^{2}\left(R ; d^{r} r\right)$. If we assume, as is the case in all the interesting examples, that $R$ is soluble, and therefore amenable, the spectral gap of $-\sum X_{j}^{2}$, which is a markovian generator is 0 . It follows that the spectral gap of $\tilde{D}_{R}$ is $C_{R}-\lambda_{D}$ and that $C_{R}=\lambda_{D}$. The conclusion is that $D_{G / K}-\lambda_{D}$ can be identified with $m_{R}^{1 / 2}\left(-\sum X_{j}^{2}\right) m_{R}^{-1 / 2}$.

An alternative way to compute $C_{R}$ is to observe that $m_{R}$ is multiplicative and therefore that $X_{j} m_{R}=\lambda_{j} m_{R}\left(\lambda_{j} \in \mathbb{R}, j=1,2, \ldots\right)$ $X_{j} m_{R}^{\alpha}=\alpha \lambda_{j} m_{R}^{\alpha},\left(\sum X_{j}^{2}\right) m_{R}^{\alpha}=\alpha^{2} \sum \lambda_{j}^{2} m_{R}^{\alpha}$ and that therefore the constant term of $m_{R}^{1 / 2}\left(-\sum X_{j}^{2}\right) m_{R}^{-1 / 2}$ is $\left(-\sum \lambda_{j}^{2}\right) / 4$. This gives, in view of the fact that $D_{R}$ has no constant term, that

$$
C_{R}=\frac{1}{4} \sum \lambda_{j}^{2}=\frac{1}{4} \rho^{2} .
$$

In the case of the Laplace-Beltrami operator on a symmetric space the above considerations amount to the standard way of computing the spectral gap in terms of the roots. Observe finally that by an easy calculation we have

$$
D_{R} m_{R}^{\alpha}=\rho^{2}\left(1 / 4-(\alpha-1 / 2)^{2}\right) m^{\alpha} .
$$

This shows that $m_{R}^{1 / 2}=\phi_{0}$ is an eigenfunction of $D_{R}$ with $D_{R} \phi_{0}=$ $\lambda_{D} \phi_{0}$ i.e. that $\phi_{0}$ is the "ground state" of the Laplace-Beltrami operator. 
Let now $G$ be an arbitrary real Lie group and let $K \subset G$ be an arbitrary compact subgroup or more generally a subgroup $K$ that contains $Z \subset K$ a central subgroup such that $K / Z$ is compact. It is then very easy to see that $K / Z$ acts by inner automorphism $\left(I_{\dot{k}}: x \rightarrow\right.$ $\left.k x k^{-1} k \in \dot{k} \in K / Z\right)$ on $G$. It follows that if $\Delta$ is an arbitrary on $G$ then $\tilde{\Delta}=\int_{K / Z} d I_{\dot{k}}(\Delta) d \dot{k}$ is $K$-bi-invariant.

A similar analysis can be done for $K$-bi-invariant convolution operators $f \mapsto f * \mu$ on $G$ (i.e. when $\mu$ is stable by the action of $I_{k}$, $k \in K)$.

Finally when $G=R \cdot K$ as in Section 2.1 the above considerations show that for $K$-bi-invariant Laplacians and $K$-bi-invariant convolution operators both Theorem A and Theorem B reduce to the analogous theorems on $R$. When $R$ is soluble and the spectral gap is zero these results have been proved in [13].

\section{Gaussian measures on groups.}

\subsection{Elementary facts on the geometry of groups.}

Let $G$ be a connected real Lie group and let $X_{1}, \ldots, X_{k}$ be left invariant fields that satisfy the Hörmander condition. These fields define therefore a left invariant distance $d(\cdot, \cdot)$ on $G, c f$. [1]. We shall always denote by $|g|_{G}=|g|=d(e, g)$. The thing to remember is that "at infinity" ||$_{G}$ only depends on $G$ and is independent of the particular choice of the fields $X_{1}, \ldots, X_{k}$. More precisely for every $e \in \Omega$ Nhd of the identity and for a new choice $X_{1}^{*}, \ldots, X_{s}^{*}$ of fields as above we have

$$
C^{-1}|g|^{\text {old }} \leq|g|^{\text {new }} \leq C|g|^{\text {old }}, \quad g \in G \backslash \Omega \text {. }
$$

It is clear of course that $|h g| \leq|h|+d(h, h g)=|h|+|g|$ and that $\left|g^{-1}\right|=d\left(e, g^{-1}\right)=d\left(g, g g^{-1}\right)=|g|$. It follows in particular that ||$h g|-| g|| \leq|h|,|| g h|-| g|| \leq|h|(g, h \in G)$ and therefore also that ||$h_{1} g h_{2}|-| g|| \leq\left|h_{1}\right|+\left|h_{2}\right|\left(g, h_{1}, h_{2} \in G\right)$.

We shall also denote by

$$
B(r)=B_{G}(r)=\{g \in G:|g| \leq r\}
$$

the corresponding $r$-ball.

Let now $H \subset G$ be some closed subgroup and let $m_{H}$ denote either the left or the right Haar measure of $H$. There exists $c>0$ then such 
that

$$
m_{H}\left\{h \in H:|h|_{G} \leq r\right\} \leq e^{c r}, \quad r>0 .
$$

Observe that the above set $H_{r}=\{h \in H:|h| \leq r\}$ is not "equivalent" with the $B_{H}(r)$ the $r$-ball in $H$. Observe also that since the involution $h \rightarrow h^{-1}(h \in H)$ interchanges the left and right Haar measure on $H$ the statement (3.1.1) need only be proved for the right measure $m_{H}$. The proof of (3.1.1) is easy. Indeed the left distance on $G$ induces | $\left.\right|_{G / H}$ a distance of the homogeneous space $\{H g: g \in G\}$ and if for every $\dot{g} \in G / H$ with $|\dot{g}|_{G / H} \leq r$ we fix as we may some $g \in \dot{g}$ with $|g| \leq 2|\dot{g}|$ we clearly have

$$
\bigcup_{|\dot{g}| \leq r} H_{r} g \subset B_{G}(3 r) .
$$

It is clear also that we can "disintegrate" $m_{G}=m_{H} \otimes m_{G / H}$ for some appropiate $C^{\infty}$-non vanishing measure on $G / H$ so that (3.1.2) gives

$$
m_{H}\left(H_{r}\right) \cdot m_{G / H}\left(B_{G / H}(r)\right) \leq m_{G}\left(B_{G}(3 r)\right)
$$

with obvious notations. $m_{G / H}$ is the Haar measure of $G / H$ if $H$ is normal but in general it does not have to be $G$-invariant. What however always holds is that $m_{G / H}\left(B_{G / H}(r)\right) \geq \varepsilon_{0}>0(r \geq 1)$ and (3.1.1) follows from the well known and obvious fact (cf. [11]) that

$$
\gamma(r)=m_{G}\left(B_{G}(r)\right) \leq C e^{c r}, \quad r>0 .
$$

What is clear also is that for any closed analytic subgroup $H \subset G$ we have $|h|_{G} \leq C|h|_{H}(h \in H)$ the best estimate the other way around is (cf. [18], [13], [43])

$$
|h|_{H} \leq C \exp \left(c|h|_{G}\right), \quad h \in H .
$$

The proof of (3.1.4) is non trivial. If $G$ is algebraic (3.1.4) follows from general considerations ( $c f$. [18]). If $G$ is simply connected soluble and $H=N$ is in the nilradical (3.1.4) was proved in [13] ( $c f$. also Section 1.6). This is the only case that will be needed in this paper. In the special case when we can write $G=H \cdot K$ where $K \Subset G$ is a compact subset we have $|h|_{H} \approx|h|_{G}(h \in H)$. (This is because for any $h, h^{\prime} \in H$ we can find $h=h_{1}, \ldots, h_{n}=h^{\prime} \in H$ such that $\left.d_{G}\left(h_{j}, h_{j+1}\right) \leq C, n \leq C d\left(h, h^{\prime}\right)\right)$. When $G=H \cdot K$ where $K$ is a 
closed group that contains $Z \subset K$ a discrete central (in $G$ ) subgroup such that $K / Z$ is compact (as in Section 2.1) and $H \cap Z=\{0\}$, we again have $|h|_{H} \approx|h|_{G}$, provided that the image of $H$ in $G / Z$ is closed. Indeed if we denote by $\pi: G \rightarrow G / Z=H \cdot(K / Z)$ the canonical projection we have from the above remark $|h|_{H} \approx|h|_{G / Z}$ but quite generally we also have $|\pi(g)|_{G / Z} \leq|g|_{G}(g \in G)$ and our result follows.

Observe finally that the above remark together with the structure theorems of Lie groups allows us to reduce the proof of (3.1.4) to the case when $G$ is soluble. That reduction is however non trivial ( $c f$. Section 4.8 and [43]). For a soluble group $G$ which we can further assume to be simply connected, the proof of (3.1.4) is done by the use of the "exponential coordinates of the second kind". One first proves that when the group $G$ of [6, Theorem 3.18.11] is nilpotent, then the coordinates $\left(t_{1}, \ldots, t_{m}\right)$ of $g \in G$ are $O\left(|g|^{N}\right)$, this is easily done by induction. We shall then choose the basis $X_{1}, \ldots, X_{m}$ in $[6$, Theorem 3.18.11] in such a way that $X_{1}, \ldots, X_{n}$ (for some $n \leq m$ ) is a basis of the nilradical. A simple use of the above special case and the results of [13] show then that, in general, the coordinates satisfy $\left|\left(t_{1}, \ldots, t_{m}\right)\right|=O(\exp (c|g|))$. From this and the proof of Theorem 3.18.12 in [6] our assertion (3.1.4) follows. The details will be left for the reader.

\subsection{Functions and measures on a group.}

Let $G$ be some real connected Lie group and let $\varphi(g) \in C^{\infty}(G)$. We shall say that $\varphi$ is an Ex-function (Ex-for "Exponential") if there exists $C>0$ such that

$$
C^{-1} \exp (-C|g|) \leq \varphi(g) \leq C \exp (C|g|), \quad g \in G,
$$

and if for any sequence of left invariant fields $X_{1}, \ldots, X_{k}, \ldots$ there exist $C_{k}, \tilde{C}_{k}>0(k \geq 1)$ such that

$$
\left|X_{1} X_{2} \cdots X_{k} \varphi(g)\right| \leq \tilde{C}_{k} \exp \left(C_{k}|g|\right), \quad g \in G
$$

Similarly we shall say that $\varphi$ is a Gs-function (Gs for "Gaussian") if there exist $C_{ \pm}, \tilde{C}_{ \pm}>0$ such that

$$
\tilde{C}_{-} \exp \left(-C_{-}|g|^{2}\right) \leq \varphi(g) \leq \tilde{C}_{+} \exp \left(-C_{+}|g|^{2}\right), \quad g \in G
$$


and for any sequence $X_{1}, \ldots, X_{k}, \ldots$ there exist $C_{k}, \tilde{C}_{k}>0(k \geq 1)$ such that

$$
\left|X_{1} \cdots X_{k} \varphi(g)\right| \leq \tilde{C}_{k} \exp \left(-C_{k}|g|^{2}\right), \quad g \in G
$$

We shall sometimes say that $\varphi$ is a strict Gs-function if for any $\varepsilon>0$ in the above estimates we can choose

$$
C_{ \pm}=\frac{1}{4 \pm \varepsilon}, \quad C_{k}=\frac{1}{4+\varepsilon}
$$

and where $\tilde{C}_{ \pm}(\varepsilon), \tilde{C}_{k}(\varepsilon)$ depend on $\varepsilon>0$. In the rest of this section we shall examine closely the above notations.

First of all it is clear that if $m_{1}, m_{2} \in \mathrm{Ex}$ (i.e. are Ex-functions) if $\varphi_{1}, \varphi_{2} \in$ Gs (i.e. are Gs-functions) if $\alpha_{1}, \alpha_{2} \in \mathbb{R} ; n_{1}, n_{2}=1,2, \ldots$, $m_{1}^{\alpha_{1}} m_{2}^{\alpha_{2}} \in \mathrm{Ex}, \varphi_{1}^{n_{1}} \varphi_{2}^{n_{2}} \in \mathrm{Gs}, m \varphi \in \mathrm{Gs}$.

Typically any positive character (e.g. the modular function $m_{G}$ ) is an Ex-function. More generally when $\left(m_{i j}\right)=M: G \rightarrow G L_{n}(\mathbb{R})$ is a group homomorphism then each matrix coefficient $m_{i j}$ is $O(\exp (C|g|))$ and satisfies (3.2.1) (This is because $m_{i j}(g x)=\sum m_{i \alpha}(g) m_{\alpha_{j}}(x)$ and the fields $X_{k}$ are left invariant).

It follows in particular that if

$$
d \mu=\varphi d g=\psi d^{r} g
$$

is a positive measure on $G$ then $\varphi \in$ Gs if and only if $\psi \in$ Gs. A measure $\mu$ as in (3.2.4) with $\varphi \in$ Gs will be called a Gs-measure.

Let now $Y$ be a right invariant field. It is clear then that $Y(g) \in$ $T_{g}(G)$ (i.e. the value of the field at $g \in G$ ) coincides with $X(g)$ the value at $g$ of the left invariant field $X$ for which $X(e)=\operatorname{Ad} g Y(e))$. The upshot is that $Y(g)=M(g)\left(X_{1}, \ldots, X_{n}\right)^{T}$ where $M(g) \in G L_{n}(\mathbb{R})$ is as above and $\left(X_{1}, \ldots, X_{n}\right)$ is a basis of left-invariant fields. From this and our previous remarks we see that in the above definition of Ex or Gs-functions we can replace left invariant fields by right invariant fields. If we use the notations

$$
\check{f}(g)=f\left(g^{-1}\right), \quad f^{h}(g)=f(g h), \quad f_{h}(g)=f(h g), \quad g, h \in G .
$$

The above considerations show that $\check{\varphi} \in \operatorname{Ex}$ (respectively $\check{\varphi} \in$ Gs) if and only if $\varphi \in$ Ex (respectively $\varphi \in$ Gs). Also if $\varphi \in$ Ex (respectively $\varphi \in \mathrm{Gs}$ ) and $k \in G$ then $\varphi^{k}, \varphi_{k} \in \mathrm{Ex}$ (respectively $\in \mathrm{Gs}$ ) and that this is so uniformly (i.e. with uniform constant) as $k \in K \Subset G$ runs through 
the compact subsets of $G$. I shall leave the reader with the task to work out which of the above observation extend to strict Gs-functions and which do not.

We shall finally need to extend the above notations to the product space $X=R \times K$ as in Section 2.1. We shall say that $\varphi \in C^{\infty}(X)$ is an Ex- (respectively Gs-) function on $X$ if and only if the functions $\varphi_{k}(r)=\varphi(r, k)$ are Ex- (respectively Gs-) functions uniformly in $k \in K$ (i.e. with uniform constants).

A typical example of a Gs-function on $R \times K$ is supplied by the convolution operator in Section 2.3 where the convolution measure $d \mu=$ $\varphi(g) d g$ is Gs. The formula (2.3.6) for $M\left(r ; k_{1}, k\right)$ and our previous remarks show that $M\left(r ; k_{1}, k\right)$ is Gs on $R$ uniformly in $k, k_{1}$ provided that $K$ is compact or more generally, uniformly when $k_{1}^{-1} k \in K_{0} \Subset K$ where $K_{0}$ is some compact subset of $K$. Indeed $m_{R}(r) \in \operatorname{Ex}$ on $R$ and $\psi(g)=\varphi\left(k_{1}^{-1} g k\right) m_{G}^{-1}(g) \in$ Gs on $G$. To show that $\left.\psi\right|_{R}$ and therefore $M$ is Gs on $R$ it suffices therefore to use Section 3.1 and the fact that when $K$ is compact we have

$$
C^{-1}|r|_{G} \leq|r|_{R} \leq C|r|_{G}, \quad r \in R,|r|_{G} \geq C .
$$

Due to the fact that $Z$ is a central subgroup, the estimate (3.2.5) also holds when $K$ is not compact ( $c f$. end of Section 3.1) provided that $k_{1}^{-1} k \in K_{0}$.

Another notion that will be used is that of a Gs $R$-left invariant positive operator $T$ on $X=R \times K$ as in Section 2.2. We shall write

$$
T=L(h, d k) \otimes\left\{* \mu_{h, k}\right\}
$$

in normal form as in Section 2.2 and we shall say that $T$ is Gs on $X$ if the measures $\mu_{h, k} \in$ Gs on $R$ uniformly in $h, k \in K$. It follows that when $K$ is compact then the operator $T$ that corresponds to a convolution operator on $G$ by a Gs measure is Gs in the above sense.

\subsection{Subgroups and quotients.}

Let $H \subset G$ be as in Section 3.1 (or at least some closed analytic subgroup for which (3.1.4) is known to hold), and let $\varphi \in$ Gs on $G$. I shall consider the restricted function $\tilde{\varphi}=\left.\varphi\right|_{H} \in C^{\infty}(H)$ by Section 3.1 it is clear that

$$
\begin{aligned}
\tilde{\varphi}(h) & \leq \tilde{C}_{+} \exp \left(-C_{+} \log ^{2}(|h|+1)\right), \\
\left|X_{1} \cdots X_{k} \tilde{\varphi}(h)\right| & \leq C_{k} \exp \left(-C_{k} \log ^{2}(|h|+1)\right), \quad h \in H .
\end{aligned}
$$


In general however $\tilde{\varphi}$ is not a Gs-function. Let us now assume throughout this section that $H$ is normal and let $\pi: G \rightarrow G / H$ be the canonical projection (For the applications that we have in mind $H \cong \mathbb{R}^{n}$, the distinction that we make below of $d^{\ell} h$ and $d^{r} h$ is therefore inessential). Let $m \in G / H$ be an Ex-function on $G / H$, then $m \circ \pi$ is an Ex-function on $G$. The analogous statement is in general false for Gs-functions. Quite generally for any $0 \leq \varphi \in C^{\infty}(G)$ we shall define (possibly $=+\infty$ )

$$
\begin{aligned}
& \varphi_{\ell}(g)=\varphi_{\ell}(\dot{g})=\int_{H} \varphi(g h) d^{\ell} h, \\
& \varphi_{r}(\dot{g})=\int \varphi(h g) d^{r} h, \quad g \in \dot{g}=g H \in G / H .
\end{aligned}
$$

We have $(\check{\varphi})_{\ell}=\left(\varphi_{r}\right)^{\vee}$. In what follows it suffices therefore to examine one of the two transforms $\varphi \rightarrow \varphi_{\ell}$ or $\varphi \rightarrow \varphi_{r}$. We shall need the following

Lemma. Let $H \subset G$ be as above. Then for every $c>\varepsilon>0$ there exists $C=C(c, \varepsilon)$ such that

$$
\int \exp \left(-c|g h|_{G}^{2}\right) d^{\ell} h \leq C \exp \left(-(c-\varepsilon)|\dot{g}|_{G / H}^{2}\right)
$$

for all $g \in G, g \in \dot{g} \in G / H$.

Proof. By Section 3.1 it is clear that we can estimate the above integral by

$$
\int_{|\dot{g}|}^{\infty} \exp \left(-c|\xi|^{2}+C \xi+C|\dot{g}|\right) d \xi .
$$

Indeed by (3.1.1) it is only matter of splitting the integral along the intersection of $g H$ with the shells $\left\{x \in G:|x|_{G} \in d \xi\right\} \subset G$. The lemma follows.

In the above lemma we can replace $|g h|_{G}$ and $d^{\ell} h$ by $|h g|_{G}$ and $d^{r} h$ and the same conclusion holds (indeed we pass from one to the other by the involution $x \rightarrow x^{-1}$ in $G$ ). With the above notations let us assume that $\varphi \in$ Gs and that $X_{1}, X_{2}, \ldots, X_{k}$ are left invariant fields and let us denote

$$
\varphi_{\ell}^{(k)}(g)=\left(\left|X_{1} \cdots X_{k} \varphi(g)\right|\right)_{\ell}, \quad \varphi_{r}^{(k)}(g)=\left(\left|X_{1} \cdots X_{k} \varphi(g)\right|\right)_{r} .
$$


It is clear from the lemma that

$$
\varphi_{\ell}^{(k)}(\dot{g}), \quad \varphi_{r}^{(k)}(\dot{g}) \leq \tilde{C}_{k} \exp \left(-C_{k}|\dot{g}|^{2}\right), \quad \dot{g} \in G / H
$$

However it is also true that

$$
\varphi_{\ell}(\dot{g}), \quad \varphi_{r}(\dot{g}) \geq \tilde{C}_{-} \exp \left(-C_{-}|\dot{g}|^{2}\right), \quad \dot{g} \in G / H .
$$

Indeed for fixed $\dot{g} \in G$ let $g \in \dot{g}$ be chosen so that $|g|_{G} \leq|\dot{g}|_{G / H}+\varepsilon$ and since with $h \in H,|h|_{H} \leq 1$ we have (cf. Section 3.1)

$$
\left.|| g h\right|_{G}-|g|_{G}|, \quad||h g|_{G}-|g|_{G} \mid \leq 1 .
$$

(3.3.5) follows by restricting the integration in (3.3.2) to the ball $|h| \leq 1$.

Let now $X$ and $Y$ be a left invariant and a right invariant field respectively on $G$ and let $\dot{X}, \dot{Y}$ the corresponding projected fields on $G / H$. It is evident (from the definition $\left.X f(g)=\lim \left(f\left(g e^{t X}\right)-f(g)\right)\right) / t$; $Y f(g)=\cdots)$ that

$$
\begin{aligned}
& \dot{X} \varphi_{r}(\dot{g})=\int_{H}(X \varphi)(h g) d^{r} h, \\
& \dot{Y} \varphi_{\ell}(\dot{g})=\int(Y \varphi)(g h) d^{\ell} h .
\end{aligned}
$$

The analogous expressions for the "multiple derivatives" $\dot{X}_{1} \dot{X}_{2} \cdots \dot{X}_{k} \varphi_{r}$ also hold. If we use this remark together with (3.3.3), (3.3.4), (3.3.5) we coclude that $\varphi_{r}, \varphi_{\ell}$ are both Gs-functions and that furthermore they are strict Gs-functions, for the quotient metric, if $\varphi$ is.

It is clear that the above considerations generalize to Gs-functions on $X=R \times K$ where for each $\varphi \in C^{\infty}(X)$ and $H \subset R$ a closed normal subgroup the corresponding functions $\varphi_{r}, \varphi_{\ell} \in C^{\infty}(R / H \times K)$ are defined in the obvious way for every slice $\varphi(\cdot, k)$ separetly.

We shall now consider more closely the restriction of Gs-function on a subgroup or more generally on a coset $g H$. Motivated by (3.3.1) we shall say quite generally that for any Lie group $H, f \in C^{\infty}(H)$ is an Sp-function (superpolynomial) with constants $c ; C_{0}, C_{1}, \cdots>0$ if

$$
\left|X_{1} \cdots X_{k} f(h)\right| \leq C_{k} \exp \left(-c \log ^{2}(|h|+1)\right), \quad h \in H, k \geq 0 .
$$

It is thus clear that the restriction to $H \subset G$ of a Gs-function on $H$ is Sp on $H$. 
More generally let $f \in$ Gs on $G$ and let us define

$$
f_{g}(h)=\left(\int_{H} f(g h) d^{\ell} h\right)^{-1} f(g h), \quad g \in G, h \in H .
$$

(One should observe that for all our applications $H$ will be in fact unimodular and $d^{r} h=d^{\ell} h$. More generally by choosing a global analytic section of $G \rightarrow G / H$, which always exists in the simply connected case, we can find an Ex-function on $G$ that allows us to pass from the $d^{r} h$ measure to the $d^{\ell} h$ measure. We shall have no use of this fact however and therefore we shall not elaborate further). Just as before if $X_{1}, \ldots, X_{k}$ are left invariant fields on $H$, which can be identified to left invariant fields on $G$, we clearly have

$$
\left(X_{1} \cdots X_{k} f\right)_{g}(h)=X_{1} \cdots X_{k}\left(f_{g}\right)(h), \quad g \in G, h \in H,
$$

and if $f \in$ Gs on $G$ by (3.3.5) we have

$$
\int f(g h) d^{\ell} h \geq C \exp \left(-C|\dot{g}|^{2}\right) .
$$

The upshot of the above consideration is that

$$
\begin{aligned}
\left|X_{1} \cdots X_{k}\left(f_{g}\right)(h)\right| & \leq \exp \left(C_{1}|g|_{G}^{2}-C_{2}|g h|_{G}^{2}\right) \\
& \leq \exp \left(c_{3}|g|_{G}^{2}-c_{4}|h|_{G}^{2}\right) .
\end{aligned}
$$

If we combine this with (3.1.4) we conclude that for every $g \in G$ the function $f_{g} \in C^{\infty}(H)$ is an Sp-function with a constant $c>0$ in (3.3.6) that only depends on $f$ and where

$$
C_{k}=C_{k}(g) \leq \tilde{C}_{k} \exp \left(c_{k}|g|^{2}\right), \quad k=0,1, \ldots
$$

The constants $c, \tilde{C}_{k}, c_{k}>0$ clearly only depend on the constants of the definition (3.2.2)-(3.2.3), and the estimates (3.3.7) are uniform for a family of functions $f$ that are uniformly Gs on $G$.

\subsection{Mass escape at infinity of the convolution product.}

Let $\mu_{j} \in \mathbb{P}(G)(j=1,2, \ldots)$ be a sequence of probability measures on $G$ that are Gs-measures uniformly in $j=1,2, \ldots$ If we bare in mind (3.1.3) we see that this implies that

$$
\mu_{j}\{g \in G:|g|>R\} \leq C \exp \left(-c R^{2}\right), \quad R>0, j=1,2, \ldots,
$$


where $C, c>0$ are independent of $R>0$ and $j=1,2, \ldots$ If we take the convolution products $\mu^{(n)}=\mu_{1} * \cdots * \mu_{n}$ we deduce

$$
\begin{aligned}
\mu^{(n)}\{g \in G:|g|>R\} & \leq \sum_{j=1}^{n} \mu_{j}\{g \in G:|g| \geq R / n\} \\
& \leq C n \exp \left(-c(R / n)^{2}\right), \quad R>0, n=1,2, \ldots
\end{aligned}
$$

We have in particular

$$
\mu^{n}\left\{g \in G:|g| \geq n^{1+\varepsilon}\right\} \leq C \exp \left(-c n^{2 \varepsilon}\right), \quad n=1,2, \ldots, \varepsilon>0 .
$$

Similarly we can consider probability measures $d \mu_{j}(g)=f_{j}(g) d g \in$ $\mathbb{P}(G)$ where $f_{j} \in \mathrm{Sp}, j=1,2, \ldots$ and where for simplicity we shall assume that $G$ is unimodular. More precisely we shall demand that there exist $c>0, C_{1}, C_{2}, \cdots>0$ such that

$$
f_{j}(g) \leq C_{j} \exp \left(-c \log ^{2}(|g|+1)\right), \quad j=1,2, \ldots, g \in G .
$$

We shall assume further that $G$ is a group of polynomial growth i.e. that

$$
\gamma(r)=\text { Haar measure of } B_{G}(r) \leq C(r+1)^{A}, \quad r>0 .
$$

It then follows that

$$
\mu_{j}\{g \in G:|g| \geq R\} \leq C C_{j} \exp \left(-c \log ^{2}(R+1)\right),
$$

for $j=1,2, \ldots, R>0$, and therefore, as before, the convolution product $\mu^{(n)}=\mu_{1} * \cdots * \mu_{n}$ satisfies

$$
\mu^{(n)}\{g \in G:|g| \geq R\} \leq C\left(\sum_{j=1}^{n} C_{j}\right) \exp \left(-c \log ^{2}(R / n+1)\right)
$$

for $R>0, n=1,2, \ldots$, with $R=n^{1+\varepsilon}$ we have in particular

$$
\mu^{(n)}\left\{g \in G:|g| \geq n^{1+\varepsilon}\right\} \leq C \sup _{1 \leq j \leq n} C_{j} \exp \left(-c \log ^{2} n\right)
$$

where $C, c>0$ are independent of $n$. 


\subsection{The Heat kernel.}

Let $\Delta_{0}=-\sum X_{j}^{2}$ be a driftless subelliptic Laplacian and let $\phi_{t}(g)$ be the corresponding convolution kernel as in Section 0. For every fixed $t>0$ the function $\phi_{t}(g)$ is then a Gs-function on $G$ (cf. [1]). In fact $\phi_{1}(g)$ is a strict Gs-function. The strict upper estimate is contained in [1]. The strict lower estimate is (implicitely) contained in [19] (especially Section 2.4, [19] II). Since we shall be able to complete the proofs of our theorems without the strict estimates, we shall not give the details here.

Let now $\Delta=-\sum_{j=1}^{n} X_{j}^{2}+X_{0}$ be a general subelliptic Laplacian (i.e. $X_{0}$ need not be zero). The convolution kernel $\phi_{t}$ is again, for every fixed $t>0$, a Gs-function. The proof of the upper estimate has been written out in a much more general context in [20]. For an alternative simple proof, ( $c f$. Section A.4). The lower estimate when $X_{0}=\sum \lambda_{i} X_{i}+\sum \lambda_{i j}\left[X_{i}, X_{j}\right]$ is an easy consequence of the scaled Harnack estimate ( $c f$. also [21]). For a general drift however this lower Gaussian estimate is difficult to prove ( $c f$. Section A.4).

From the above and the considerations at the end of Section 3.2 we see that $T$ the left invariant operators on $R \times K$ that corresponds to the semigroup $T_{t}=e^{-t \Delta}$ on $G$ as in sections 2.5, 2.6, 2.7 are Gs-operators when $K$ is compact. This statement remains true in general, even when $K$ is not compact, but this statement is not trivial to prove. Since we shall be able to do without this general case we shall not give this proof here.

\section{Upper estimates.}

\subsection{Gaussian measures on a special class of groups.}

In this section we shall consider a real Lie group $G$ and $H \subset G$ a closed normal subgroup that satisfy the following conditions: $H \cong$ $\mathbb{R}^{n}$ and $G / H \cong V \times S$ where $V \cong \mathbb{R}^{m}$ and $S$ is compact. We shall summarize this information in the exact sequence

$$
0 \longrightarrow H \cong \mathbb{R}^{n} \longrightarrow G \underset{p}{\longrightarrow} G / H \cong V \times S \cong \mathbb{R}^{m} \times S \longrightarrow 0
$$

The above situation is not as special as it looks. Indeed let $\bar{G}$ be simply connected group and let $N \subset Q \subset \bar{G}$ be its radical and nilradical. Let 
further $[N, N]$ the analytic subgroup that corresponds to $[\mathfrak{n}, \mathfrak{n}]$ where $\mathfrak{n} \subset \overline{\mathfrak{g}}$ is the nilradical of the Lie algebra of $\bar{G}$. Then the group $G=$ $\bar{G} /[N, N]$ satisfies the above conditions (4.1.1) with $H=N /[N, N]$, $V=Q / N$ and $S=\bar{G} / Q . S$ is a semisimple group. When $\bar{G}$ is soluble we have $S=\{e\}$. When $\bar{G}$ is amenable (e.g. when $\overline{\mathfrak{g}}$ is an R-algebra) then $S$ is compact. Observe that if we assume in addition that $\overline{\mathfrak{g}}$ is an algebraic algebra (i.e. that it is the Lie algebra of some algebraic Lie group) in the above situation we have $\bar{G} /[N, N]=G=H \wedge(V \times S)$ (cf. [7]) and if $\bar{G}=Q$ is soluble we have $Q /[N, N]=H<V$.

The basic thing to observe is that under the condition (4.1.1) the group $G / H\left(\cong \mathbb{R}^{m} \times S\right)$ can be made to act naturally on $H$ so as to have

$$
\pi: G / H \longrightarrow G L(H) \text {. }
$$

This is of course true in general (and trivially so) when $G \cong H<G / H$ (e.g. $G$ simply connected and $H=Q$, the radical of $G$ ) but here the action (4.1.2) is obtained from inner automorphisms because $H$ is abelian. Indeed for $x \in G / H$ we choose some $g \in G$ such that $p(g)=x$ and then the action $h \rightarrow g^{-1} h g$ is independent of the particular choice of $g$.

The Lie algebra of $V$ and $H$ will be identified with $V$ and $H$ respectively and we shall consider

$$
d \pi: V \longrightarrow \mathfrak{g l}(H)
$$

We shall also consider the roots of the action (4.1.3) which are $\lambda \in$ $\operatorname{Hom}_{\mathbb{R}}[V ; \mathbb{C}]$ and are defined by

$$
(d \pi(v)-\lambda(v)) w=0, \quad v \in V,
$$

and some $0 \neq w \in H \otimes_{\mathbb{R}} \mathbb{C}$. The corresponding root spaces $U_{\lambda} \subset H \otimes_{\mathbb{R}} \mathbb{C}$ are defined accordingly.

I shall defined then $L_{1}, L_{2}, \ldots, L_{p}$ all the distinct real parts $(L=$ $\operatorname{Re} \lambda$ ) of these roots (contrary to what was done in Chapter 1 the zero real part is also admitted here). If then $H_{j} \subset H$ is defined by the fact that $H_{j} \otimes \mathbb{C}=\sum U_{\lambda}$ for all the $\lambda$ 's such that $\operatorname{Re} \lambda=L_{j}$ we obtain $H=H_{1} \oplus \cdots \oplus H_{p}$ a decomposition of $H$ as a direct sum of subspaces. All the subspaces $H_{j}$ are stable by the representation (4.1.2) and are such that if $G / H$ э $\dot{g}=(v, s)(v \in V, s \in S)$ we have

$$
\operatorname{det}\left(\pi_{j}(\dot{g})\right)=e^{d_{j} L_{j}(v)},
$$


where $d_{j}=\operatorname{dim} H_{j}$ and $\pi_{j}=\pi \mid H_{j}$ (This is because the determinant is real and its modulus is clearly given by (4.1.4)). Observe also that in the relevant cases $S$ is compact or semisimple and therefore $\operatorname{det} \pi(s)=1$, $s \in S$. All the above facts are consequences of elementary linear algebra and will thus be left for the reader.

It is of course clear that if $G$ satisfies (4.1.1) then we can represent

$$
0=\sum_{j=1}^{p} \alpha_{j} L_{j}, \quad \alpha_{j} \geq 0
$$

non trivially (i.e. not all $\alpha_{j} L_{j}=0$ in the above sum) if and only if $\mathfrak{g}$ is a C-algebra.

Proposition. Let $G$ be a real Lie group that satisfies the conditions (4.1.1) and let us assume that the Lie algebra $\mathfrak{g}$ satisfies the $\mathrm{C}$-condition. Let $\mu_{j} \in \mathbb{P}(G), j=1,2, \ldots$ be a sequence of probability measures and let us assume that $\mu_{j} \in$ Gs on $G$ uniformly in $j$ (i.e. with constants that are independent of $j$ ). Let further $\mu^{(n)}=\mu_{1} * \cdots * \mu_{n}$ be the corresponding convolution products.

Then there exists $c>0$ such that for every $f \in C_{0}^{\infty}$ we have

$$
\left\langle\mu^{(n)}, f\right\rangle=O\left(e^{-c n^{1 / 3}}\right), \quad n \geq 1 .
$$

In fact we have $d \mu^{(n)}(g)=\varphi^{(n)}(g) d g$ where $\varphi^{(n)} \in C^{\infty}(G)$ (and even $\varphi^{(n)} \in$ Gs on $G$ but this is irrelevant) and "morally" what the estimate (4.1.5) actually says is

$$
\varphi^{(n)}(g)=O\left(e^{-c n^{1 / 3}}\right), \quad g \in G .
$$

The proof of (4.1.6) will be given in Section 4.5.

\subsection{The Fourier transform.}

$G \supset H$ and all other notations will be as in Section 4.1. We shall consider $d \mu(g)=\varphi(g) d g$ a Gs-probability measure on $G$ and define

$$
\theta_{\dot{g}}(h)=\left(\int_{H} \varphi(g h) d h\right)^{-1} \varphi(g h), \quad h \in H, g \in \dot{g} \in G / H,
$$


the above function will be identified with a function $\theta_{\dot{g}}(x) \in C^{\infty}\left(\mathbb{R}^{n}\right)$ uniquely defined up to translation $\cdot \mapsto \cdot+\alpha$ on $\mathbb{R}^{n}\left(\alpha \in \mathbb{R}^{n}\right)$. The modulus of the Fourier transform

$$
f_{\dot{g}}(\xi)=\left|\hat{\theta}_{\dot{g}}(\xi)\right|
$$

is therefore uniquely defined. For the sequence of measures given in the proposition of Section 4.1 we shall consider $d \mu_{j}(g)=\varphi_{j}(g) d g$ $(j=1,2, \ldots)$ and the corresponding $f_{g}(\xi)=f_{j, \dot{g}}(\xi)=\left|\hat{\theta}_{g}(\xi)\right|$ (for typographical reasons we shall drop the $j=1, \ldots$ and the "dot" above the $g$ ). We shall need the following

Lemma. Let $\hat{H}=\hat{H}_{1} \oplus \cdots \oplus \hat{H}_{p}$ be the dual decomposition of $H=$ $H_{1} \oplus \cdots \oplus H_{p} \cong \mathbb{R}^{n}$ and let $\xi=\left(\xi_{1}, \ldots, \xi_{p}\right) \in \hat{H}, \xi_{i} \in \hat{H}_{i}, i=1, \ldots, p$ be the corresponding coordinates. Then (uniformly in $j=1,2, \ldots$ ) we can find functions $f_{g}^{(1)}, \ldots, f_{g}^{(p)},(g \in G / H)$ (i.e. these functions are independent of $j=1,2, \ldots)$ that satisfy

$$
f_{g}(\xi) \leq f_{g}^{(1)}\left(\xi_{1}\right) \cdots f_{g}^{(p)}\left(\xi_{p}\right), \quad g \in G / H, \xi=\left(\xi_{1}, \ldots, \xi_{p}\right) \in \hat{H},
$$

$$
0 \leq f_{g}^{(i)} \leq 1, \quad \int_{\hat{H}_{i}} f_{g}^{(i)}(\xi) d \xi \leq C e^{c|g|^{2}}
$$

for all $g \in G / H, i=1,2, \ldots, p$.

Proof. It is clear that $0 \leq f_{g}(\xi) \leq 1$ (for all $j=1, \ldots$ ) and we shall presently show that for all $N \geq 1$ there exists $C, c>0$ such that (again for $j=1, \ldots$ )

$$
f_{g}(\xi) \leq C e^{c|g|^{2}}|\xi|^{-N}, \quad \xi \in \hat{H}, g \in G / H
$$

Let $N$ be so large that there exist $0 \leq f^{(i)}\left(\xi_{i}\right) \leq 1, i=1,2, \ldots, p$ such that

$$
\begin{gathered}
\min \left\{1,|\xi|^{-N}\right\} \leq f^{(1)}\left(\xi_{1}\right) \cdots f^{(p)}\left(\xi_{p}\right), \quad \xi=\left(\xi_{1}, \ldots, \xi_{p}\right) \in \hat{H} \\
\int_{\hat{H}_{i}} f^{(i)}(\xi) d \xi<+\infty, \quad i=1,2, \ldots, p .
\end{gathered}
$$


We can for instance take $f^{(i)}(\xi)=\min \left\{1,|\xi|^{-2 d}\right\}$ where $d=\sum d_{i}$ and $N=2 p d$. Then by rescaling we have

$$
\min \left\{1, C e^{c|g|^{2}}|\xi|^{-N}\right\} \leq f^{(1)}\left(C e^{-c|g|^{2}} \xi_{1}\right) \cdots
$$

(with a different $C$ and $c$ ) and our lemma follows with $f_{g}^{(i)}(\xi)=$ $f^{(i)}\left(C e^{-c|g|^{2}} \xi\right)$.

The estimate (4.2.2) is clearly implied by

$$
\int\left|\frac{\partial^{k}}{\partial x_{i_{1}} \cdots \partial x_{i_{k}}} \theta_{g}(x) d x\right| \leq C e^{c|g|^{2}},
$$

$g \in G / H$ uniformly in $j$, with $k=1, \ldots$ (and $C=C_{k}, c=c_{k}$ ). To prove (4.2.3) let $X_{1}, \ldots, X_{s}$ be a basis of left invariant fields on $G$. It is clear then, by induction on $k$, that $\left(\partial^{k} / \partial x_{i_{1}} \cdots \partial x_{i_{k}}\right) \varphi(g x)$ $\left(x \in H \cong \mathbb{R}^{n}, g \in G\right.$ ) is a linear combination of expressions of the form $\left(X_{j_{1}} \cdots X_{j_{r}} \varphi\right)(g x)(x \in H, g \in G)$ our estimate (4.2.3) therefore follows from results in Section 3.3.

The above proof shows in fact that if the original $\varphi$ is a strict Gsfunction on $G$ then in (4.2.3) (and therefore in (4.2.2) and in (4.2.1)) we can choose $c=\varepsilon>0$ arbitrary small provided that the $C=C_{k}$ of (4.2.3) (and the other corresponding $C$ 's in (4.2.2) and (4.2.1)) are made to depend on $\varepsilon>0$. When we are considering several $\varphi_{j}(j \geq 1)$ the above strict-Gs property can of course to be made uniform in $j$.

\subsection{The disintegration of the kernel.}

In this section I shall follows closely [13, I, Section 3]. I shall consider $H \subset G$ as in Section 4.1 with $H \cong \mathbb{R}^{a}$ (notice that to avoid a possible confusion with notations that I followed, I have changed here the dimensions of $H$ into $a: \operatorname{dim} H=a$ and not $n$ as in the previous sections) and shall assume that $V \cong \mathbb{R}^{m}$ is a vector subgroup and $S$ is compact. I shall disintegrate $\mu_{j}$ for $j=1,2, \ldots$

$$
\mu_{j}=\int_{G / H} \nu_{\dot{g}}^{(j)} d \check{\mu}_{j}(\dot{g}),
$$

where $\nu_{\dot{g}}^{(j)}$ are probability measures on the fibers $g H=\dot{g} \in G / H$ (all the other notations are as before). From this it clearly follows that

$$
\mu^{(n)}=\int_{G / H} \cdots \int_{G / H} \nu_{g_{1}} * \cdots * \nu_{g_{n}} d \check{\mu}_{1}\left(g_{1}\right) \cdots d \check{\mu}_{n}\left(g_{n}\right),
$$


where the $*$ indicates convolution in $G$. I shall now identify, as I may, $\nu_{g}$ with a measure on $H$ (by $H \leftrightarrow g H$ up to translation on $H$ ), and, for any $\nu \in \mathbb{P}(H)$ and $g \in G / H$, I shall denote by $\nu^{g} \in \mathbb{P}(H)$ the image of $\nu$ by the action $\pi: G / H \rightarrow \operatorname{Aut}(H)$ on $H$ (induced by inner automorphisms as in Section 4.1). It is clear then that the integrand of (4.3.1) which, up to translation, can be identified to a measure on the coset $\dot{g}_{1} \cdots \dot{g}_{n} \subset G$, can also be identified up to translation with

$$
\nu\left(g_{1}, \ldots, g_{n}\right)=\nu_{g_{1}}^{s_{1}} * \nu_{g_{2}}^{s_{2}} * \cdots * \nu_{g_{n}}^{s_{n}} \in \mathbb{P}(H),
$$

where $s_{j}=g_{1} \cdots g_{j} \in G / H$ and where the convolution product is now taken in $H$. (This identification is now done for the "right product" identification $\left.H \leftrightarrow H s_{n}\right)$. Now the measures $\nu\left(g_{1}, \ldots, g_{n}\right)$ can be identified to a $L^{\infty}(H)$ functions of $H$ and, since convolution goes by Fourier transforms to pointwise product, we have

$$
\left\|\nu\left(g_{1}, \ldots, g_{n}\right)\right\|_{\infty} \leq \int_{\hat{H}} f_{g_{1}}\left(\pi\left(s_{1}\right)^{*} \xi\right) \cdots f_{g_{n}}\left(\pi\left(s_{n}\right)^{*} \xi\right) d \xi .
$$

Note that, to simplify notations I have dropped throughout from the $\nu$ 's and the $f$ 's the $j=1,2, \ldots$ coming from $\mu_{j}$. To estimate the integral in (4.3.2) I shall first use the decomposition $\hat{H}=\hat{H}_{1} \oplus \cdots \oplus \hat{H}_{p}$ coming from $L_{j}=\operatorname{Re} \lambda(j=1, \ldots, p)$ the real parts of the roots of the representation $\pi: G / H \rightarrow G L(H)$ as in Section 4.1. For the above decomposition and with the obvious notation $\xi=\left(\xi_{1}, \ldots, \xi_{p}\right) \in \hat{H}, \mathrm{I}$ shall apply the Lemma of Section 4.2 and estimate

$$
\left|f_{g}(\xi)\right| \leq f_{g}^{(1)}\left(\xi_{1}\right) \cdots f_{g}^{(p)}\left(\xi_{p}\right), \quad g \in G / H
$$

This estimate will be inserted in the integrand of (4.3.2). It follows that the right hand side of (4.3.2) can be estimated by

$$
\inf \int_{\hat{H}} f_{g_{j_{1}}}^{(1)}\left(\pi\left(s_{j_{1}}\right)^{*} \xi_{1}\right) f_{g_{j_{2}}}^{(2)}\left(\pi\left(s_{j_{2}}\right)^{*} \xi_{2}\right) \cdots f_{g_{j_{p}}}^{(p)}\left(\pi\left(s_{j_{p}}\right)^{*} \xi_{p}\right) d \xi
$$

where the infimum is taken over all choices $1 \leq j_{i} \leq n(i=1, \ldots, p)$. The integral under the above inf splits in $\hat{H}_{1} \oplus \cdots \oplus \hat{H}_{p}$ and each integral $\int_{\hat{H}_{j}}$ can be explicitely computed by a change of variable whose determinant is known by (4.1.4).

Let us introduce the following notation $s_{j}=\left(b_{j}, \sigma_{j}\right), g_{j}=\left(X_{j}, \tilde{\sigma}_{j}\right)$ $\in V \times S,(j=1, \ldots, n)$ and for each $g=(u, \sigma) \in G / H$ let us observe 
that $|g|_{G / H} \approx|u|_{V}=$ the norm in $V$ (provided that $|g| \geq 1$ ) let us further denote by

$$
A_{n}\left(L_{i}\right)=\inf _{1 \leq j \leq n} \exp \left(c\left|X_{j}\right|^{2}-d_{i} L_{i}\left(b_{j}\right)\right)
$$

With these notations if we combine all the above estimates we obtain

$$
\left\|\nu\left(g_{1}, \ldots, g_{n}\right)\right\|_{\infty} \leq C A_{n}\left(L_{1}\right) \cdots A_{n}\left(L_{p}\right)
$$

\subsection{The probabilistic estimate.}

All the notations introduced up to now will be preserved. The $X_{j} \in$ $V \cong \mathbb{R}^{m}(j=1,2, \ldots)$ in the definition of $A_{n}\left(L_{i}\right)$ will be independent (not necessarily equidistributed) random variables such that the corresponding density functions $\mathbb{P}\left[X_{j} \in d x\right]=\psi_{j}(x) d x$ are Gs-functions on $\mathbb{R}^{n}$ uniformly in $j=1,2, \ldots$. We have then $b_{t}=X_{1}+\cdots+X_{t}$. In Section $B$ of the appendix we shall prove the estimate

$$
\mathbb{E}\left(A_{n}\left(L_{1}\right) \cdots A_{n}\left(L_{p}\right)\right)=O\left(\exp \left(-c n^{1 / 3}\right)\right)
$$

for some $c>0$ provided that the real roots $L_{1}, \ldots, L_{p}$ satisfy the C-condition $(c f .(4.1 .5))$. This estimate was proved in [13] when all the $X_{j}$ 's are equidistributed centered Gaussian variables (so that $b_{t}=$ $b(t)=X_{1}+\cdots+X_{t} \in \mathbb{R}^{m}$ is brownian motion) and when the constant $c>0$ appearing in the definition of $A_{n}\left(L_{i}\right)$ (cf. (4.3.3)) is small enough. Here again, if we are prepared to use the fact that for a driftless Laplacian the heat kernel on $G$ is a strict Gs-function, we can suppose that the $c>0$ in (4.3.3) is as small as we like. In the appendix however we shall prove (4.4.1) without that restriction.

\subsection{The proof of the Proposition of Section 4.1.}

All our previous notations are preserved. Let $0 \leq \varphi \in C_{0}^{\infty}(G)$ and let

$$
\sup _{g \in G} \int_{H} \varphi(h g) d h=C_{0} \text {. }
$$


(I implicitely use here the right identification $H \leftrightarrow H g$ !). Then the basic formula (4.3.1) that expresses $\mu^{(n)}$ as a barycenter of measures sitting in the various cosets $H g$ gives

$$
\left\langle\mu^{(n)}, \varphi\right\rangle \leq C_{0} \int\left\|\nu\left(g_{1}, \ldots, g_{n}\right)\right\|_{\infty} d \check{\mu}_{1}\left(g_{1}\right) \cdots d \check{\mu}_{n}\left(g_{n}\right),
$$

where by Section $3.3 \check{\mu}_{j} \in \mathbb{P}(G / H)(j=1, \ldots)$ are Gs-measures on $G / H$ (uniformly in $j$ ). Since $G / H=V \times S$, we can project $\check{\mu}_{j}$ on $V$ by the canonical $G / H \rightarrow V$ and obtain a sequence of probability measures $\psi(x) d x(j=1,2, \ldots)$ on $\mathbb{R}^{m}$ that are uniformly Gs on $\mathbb{R}^{m}$. A sequence $X_{j} \in \mathbb{R}^{m}(j=1,2, \ldots)$ of independent random variables can then be defined by $\mathbb{P}\left[X_{j} \in d x\right]=\psi_{j}(x) d x$. The corresponding $A_{n}\left(L_{i}\right)$ can thus be constructed and because of (4.3.4) we clearly have

$$
\begin{aligned}
\int\left\|\nu\left(g_{1}, \ldots, g_{n}\right)\right\|_{\infty} & d \check{\mu}_{1}\left(g_{1}\right) \cdots d \check{\mu}_{n}\left(g_{n}\right) \\
\leq C \mathbb{E}\left(A_{n}\left(L_{1}\right) \cdots A_{n}\left(L_{p}\right)\right) &
\end{aligned}
$$

The estimate (4.1.6) follows from (4.4.1) and (4.5.1).

\subsection{The Proposition for an arbitrary soluble group.}

In this section I shall prove the following

Proposition. Let $Q$ be a connected soluble group that satisfies the $\mathrm{C}$ condition. Let $\mu_{j} \in \operatorname{Gs}(Q) \cap \mathbb{P}(Q)(j=1,2, \ldots)$ uniformly in $j$ and let $\mu^{n}=\mu_{1} * \cdots * \mu_{n}$. There exists then $c>0$ such that

$$
\left\langle\mu^{(n)}, f\right\rangle=O\left(\exp \left(-c n^{1 / 3}\right)\right), \quad f \in C_{0}^{\infty}(Q) .
$$

We shall need the following

Lemma. Let $G$ be an arbitrary connected real Lie group and let $K \subset G$ be some closed subgroup.

i) If the conclusion of the proposition is valid for $G / K$ then it is also valid for $G$.

ii) Conversely if we assume that $K$ is compact and assume that the conclusion of the proposition is valid for $G$ it is also valid for $G / K$. 
The proof of the lemma is evident and will be left to the reader.

The first step in the proof of the proposition is to reduce the proof to the case when the center of $\mathfrak{q}$ is 0 . To see this let $\mathfrak{z} \subset \mathfrak{q}$ and let more generally $\mathfrak{z}=\mathfrak{z}_{0} \subset \mathfrak{z}_{1} \subset \cdots \subset \mathfrak{z}_{j} \subset \cdots \subset \mathfrak{q}$ be defined inductively by $\mathfrak{z}_{j}=\pi_{j}^{-1}$ (the center of $\mathfrak{q} / \mathfrak{z}_{j-1}$ ) where $\pi_{j}: \mathfrak{q} \rightarrow \mathfrak{q} / \mathfrak{z}_{j-1}$ is the canonical projection. Then clearly $\mathfrak{p}=\cup \mathfrak{z}_{j}$ (in fact $\mathfrak{z}_{k+1}=\mathfrak{z}_{k}$ for some $k$ ) is a nilpotent ideal and $\mathfrak{p} \subset \mathfrak{n}$ and, by its construction, $\mathfrak{q} / \mathfrak{p}$ has trivial center. An easy composition series argument on $\mathfrak{q}$ shows that $\mathfrak{q} / \mathfrak{p}$ is a C-algebra.

Let $Z_{j} \subset Q$ be the analytic subgroup that corresponds to $\mathfrak{z}_{j}$. One easily sees by induction that these are closed subgroups. Indeed quite generally if $Z$ is the analytic subgroup that corresponds to the center of the algebra $Z$ is closed for $\bar{Z}$, its closure, is connected and the subalgebra $\overline{\mathfrak{z}}$ that corresponds to $\bar{Z}$ is central. To make the required reduction therefore it suffices to consider $P$ the analytic subgroup that corresponds to $\mathfrak{p}$ and to consider $Q / P$. Our reduction then follows from the lemma.

Let now $\theta: \tilde{Q} \rightarrow Q$ be the universal covering map and let $\tilde{N} \subset \tilde{Q}$ be the nilradical. Our hypothesis that the center of $\mathfrak{q}$ is trivial implies then that

$$
\operatorname{Ker} \theta \cap \tilde{N}=\{e\} .
$$

Indeed $\Theta=\tilde{N} \cap \operatorname{Ker} \theta$ is a discrete central subgroup of $\tilde{Q}$ and therefore $\Theta \subset Z_{N}$, the center of $\tilde{N}$ which can be identified with a vector space $Z_{N} \cong \mathbb{R}^{c}(c \geq 1)$. The Ad action induces $\operatorname{Ad}: \tilde{Q} \rightarrow G L\left(Z_{N}\right)$ and if we denote by $V_{Z} \subset Z_{N}$ the vector subspace generated by $\Theta$ we have $\left.\operatorname{Ad}(Q)\right|_{V_{Z}}=\mathrm{Id}$. This means that $V_{Z} \subset \mathfrak{q}$ is central and therefore $V_{Z}=\{0\}$ by our hypothesis. (4.6.1) follows.

To finish the proof it suffices to make one further reduction. Indeed let $\tilde{N} \subset \tilde{Q} ; N \subset Q$ be the corresponding closed nilradicals, i.e. the analytic subgroups that correspond to the nilradical $\mathfrak{n} \subset \mathfrak{q}$. By (4.6.1) the mapping $\theta_{N}: \tilde{N} \rightarrow N$ is then (1-1), continuous and onto. It therefore is a homeomorphism. $N$ is therefore simply connected and therefore $N_{2} \subset N$, the analytic subgroups that correspond to $[\mathfrak{n}, \mathfrak{n}]$, is closed. By our lemma we can reduce the proof of our proposition to the group $Q / N_{2}=G$. This new group satisfies (4.1.1) with $H=N / N_{2} \cong$ $\tilde{N} / \tilde{N}_{2} \cong \mathbb{R}^{a}$ (by 4.6.1). Indeed $G / H \cong Q / N$ is a homomorphic image of $\tilde{Q} / \tilde{N} \cong \mathbb{R}^{d}$ and has therefore the required form $G / H \cong \mathbb{R}^{m} \times \mathbb{T}^{b}=$ 
$\mathbb{R}^{m} \times S$ as in (4.1.1). The condition (4.1.5) is clearly verified by the Ccondition on our original group and the proof of our proposition follows from the lemma and the proposition in Section 4.1.

The above proof gives "for free", so to speak, something slightly stronger. What it shows is that the conclusion of the proposition also holds for any amenable group (i.e. when $\mathfrak{g} / \mathfrak{q}=\mathfrak{s}$ is a compact semisimple algebra). This proposition can therefore be viewed as a generalization of the results of [13]. Indeed let $G$ be such a group and let $Q$, $S \subset G$ be the radical and some Levi subgroup respectively. Then since $S$ is compact we can form the canonical semidirect product and the canonical covering map $\pi: Q \wedge S=\tilde{G} \rightarrow G$ which is now an isogeny (i.e. $\operatorname{Ker} \pi$ is a finite subgroup). By our lemma again, it suffices to prove the proposition for the group $\tilde{G}$. For the group $\tilde{G}=Q \wedge S$ if we repeate our previous argument we reduce the proof to the case where $\mathbb{R}^{n} \cong N=H \subset \tilde{G}$ and $\tilde{G} / N=Q / N \times S=\mathbb{R}^{m} \times S$ (cf. [13]). This completes the proof.

\subsection{General Lie groups.}

The key to the proof of Theorem B for a general connected real Lie group is to show that with the machinery that we have developed we can give a proof of that theorem for groups of the form $G=Q \wedge S$ where $Q$ is soluble and connected and where $S$ is semisimple. Indeed for such a $G$, as we already pointed out ( $c f$. Section 2.1), there exists $Z \subset S$ a discrete subgroup that is central in $G$ and of finite index in the center of $S$. Let $G_{1}=G / Z=Q \wedge S_{1}=Q \wedge(S / Z)$. Then $G_{1}$ is a similar group but has the additional property that the center of $S_{1}$ is finite. We can therefore write $G_{1}=Q N A K=R K$ where $N A K=S_{1}$ is the Iwasawa decomposition of $S_{1}, K$ is compact and $R$ is soluble.

The proof of Theorem B for the group $G_{1}$ is contained in Section 2.4. Indeed if we identify $f \mapsto f * \mu$ with an R-left invariant operator on $X=R \times K$ we see that we have our theorem as long as we can show that (2.4.7) in Section 2.4 holds with $\theta(n)=\exp \left(-c n^{1 / 3}\right)$. But modulo Section 3.5 this is exactly what was proved in Section 4.6.

To complete the proof of Theorem B for the group $G$ we shall use the following general observation. Let quite generally $\pi: G \rightarrow G_{1}$ be some covering map between two arbitrary Lie groups and let $d \mu(g)=$ $\phi(g) d g$ be some Gs-probability measure on $G$. Let the corresponding 
image measure be $\mu_{1}=\check{\pi}(\mu) \in \mathbb{P}\left(G_{1}\right)$. $\mu_{1}$ as we saw in Section 3 , is a Gs-measure on $G_{1}$ and can be written

$$
\begin{aligned}
d \mu_{1}\left(g_{1}\right) & =\phi_{1}\left(g_{1}\right) d g_{1} \\
\phi_{1}\left(g_{1}\right) & =\sum_{z \in \operatorname{Ker} \pi} \phi(g z), \quad g_{1}=g \operatorname{Ker} \pi \in G_{1} .
\end{aligned}
$$

The obvious observation is that if Theorem B holds for $\mu_{1}$ on $G_{1}$ then it also holds for $\mu$ on $G$. This is because of the amenability of $\operatorname{Ker} \pi \subset G$, which implies that if we denote by $\|\cdot\|_{2 \rightarrow 2}$ the $f \mapsto f * \cdot$ convolution norms on $L^{2}\left(G ; d^{r} g\right)$ and $L^{2}\left(G_{1} ; d^{r} g_{1}\right)$ we have

$$
\|\mu\|_{2 \rightarrow 2}=\left\|\mu_{1}\right\|_{2 \rightarrow 2} \text {. }
$$

(4.7.2) is very well known. Observe also that Section 2.4 in fact contains a proof (4.7.2).

Let now $G$ be an arbitrary connected real Lie group not necessarilly of the form $Q \wedge S$ and let $Q \subset G$ be its radical let further $S \subset G$ be some Levi subgroup that is an analytic but not necessarily closed subgroup of $G$. It is clear that $Q \cap S$ is a closed subgroup of $Q$ and a central subgroup of $S$ (Indeed $Q \cap S$ is a normal and discrete subgroup of $S$ for the intrinsic Lie topology of $S$ ). As already pointed out twice before there exists then $Z_{1} \subset Q \cap S$ a discrete central subgroup of $G$ that is of finite index in $Q \cap S$. We shall quotient by $Z_{1}$ and obtain $G_{1}=G / Z_{1}$. This group has a Levi decomposition $G_{1}=Q_{1} S_{1}$ as before with the additional property that $Q_{1} \cap S_{1}$ is finite. By what was said just above, if we can prove our theorem for $G_{1}$ then we also have it for $G$.

Using the canonical action of $S_{1}$ on $Q_{1}$ we can then construct the semidirect product $\tilde{G}=Q_{1} \wedge S_{1}$ where the kernel of the canonical projection $\tilde{G} \rightarrow G_{1}$ is finite. Since we already know that the Theorem holds for $\tilde{G}$ and since the summation in (4.7.1) is finite, it follows that the Theorem holds also for $G_{1}$ (here we make essential use of the fact that Theorem can be stated equivalently either as $\mu^{(n)}(e)=O(\cdots)$ or $\mu^{(n)}(g)=O(\cdots)$ for any $\left.g \in G\right)$. The proof of Theorem B is complete.

\subsection{The Iwasawa radical revisited.}

It is interesting to observe that the techniques of the previous section prove the following 
Proposition. Let $G$ be a connected real Lie group. Then $R \subset G$, the analytic subgroup that corresponds to $\mathfrak{r}=\mathfrak{q}+\mathfrak{n}_{S}+\mathfrak{a} \subset \mathfrak{g}$, an Iwasawa radical of the Lie algebra (cf. Chapter 1 ), is closed.

Indeed let $Q \subset G$ the (closed) radical of $G$ and let $\Sigma \subset G$ some analytic (but not necessarily closed) Levi subgroup. Let $\Sigma=N A K$ be some Iwasawa decomposition of $\Sigma$. Then $Z_{0}=Q \cap \Sigma \subset \Sigma$ is a discrete (for the intrinsic Lie-group topology of $\Sigma$ ) central subgroup of $\Sigma$ therefore $Z_{0} \subset Z(\Sigma) \subset K$ where $Z(\Sigma)$ is the center of $\Sigma$. It follows in particular that $Q \cap A N=\{e\}$. Let us form $Q \wedge \Sigma=\tilde{G}$ the semidirect product and let $\theta: \tilde{G} \rightarrow G$ be the canonical covering map. Let $\pi: \tilde{G} \rightarrow \Sigma$ be the canonical projection so that

$$
\pi(\operatorname{Ker} \theta)=Z_{0}
$$

The subgroup $R=Q A N \subset G$ is the image by $\theta$ of the subgroup $\tilde{R}=Q \wedge A N \subset \tilde{G}$. Clearly

$$
\tilde{R} \cap \operatorname{Ker} \theta=\{e\}
$$

and to show that $R$ is closed it suffices to show that if $k_{n} \in \operatorname{Ker} \theta$ $(n \geq 1)$ is a a sequence that satisfies $d_{\tilde{G}}\left(k_{n}, \tilde{R}\right) \rightarrow 0$ then $k_{n}=e$ for all $n \geq n_{0}$ large enough. The proof of this is easy. Indeed we have $d_{\Sigma}\left(\pi\left(k_{n}\right), A N\right) \rightarrow 0$ and therefore (since $\pi\left(k_{n}\right) \in Z(\Sigma)$ which is a discrete subgroup of $\Sigma) \pi\left(k_{n}\right)=e, n \geq n_{0}$. Our assertion therefore follows from (4.8.1) and the fact that $\operatorname{Ker} \pi=Q$.

We shall say that the subgroup $R \subset G$ is an Iwasawa radical of $G$. As we already pointed out for an arbitrary group $G$ we can find $Z \subset G$ some central discrete subgroup such that $G_{1}=G / Z$ is such that $Q_{1}$ its radical and $\Sigma_{1}$ some Levi subgroup have a finite intersection (i.e. $\left.\left|Q_{1} \cap \Sigma_{1}\right|<+\infty\right)$. By quotienning further by $Z_{1} \subset G_{1}$ another central discrete subgroup we can obtain $G_{2}=G_{1} / Z_{1}=Q_{2} \Sigma_{2}$ where $Q_{2}$ is the radical of $G_{2}$ and $\Sigma_{2}$ is semisimple with finite center. But then clearly $G_{2}=R K$ where $R$ is an Iwasawa radical and $K$ is a compact subgroup. 


\section{The proof of the Theorem (NB) and the lower estimates.}

\subsection{The proof of Theorem $A_{2}$ for a special class of groups.}

In this section $G$ will be a real Lie group that can be written in the form $G=R \cdot K$ where the closed subgroup $R$ is a simply connected soluble NC-group and $K$ is a compact subgroup such that $R \cap K=$ $\{e\}$. We shall identify $G$ with $X=R \times K$ as in Section 2.1 and then decompose

$$
R=N \wedge Q
$$

as in sections 1.3-1.6 where $N$ is a simply connected nilpotent subgroup and $Q$ is a simply connected R-group. I shall furthermore systematically use the following notation for the "coordinates" in $X$

$$
x=(r, k)=(n, q, k),
$$

for $x \in X=G, r \in R, n \in N, q \in Q, k \in K$. I shall fix $\Delta=-\sum X_{j}^{2}$ some driftless Laplacian on $G$ and $\lambda \geq 0$ will denote the corresponding spectral gap. On the space $X$, I shall consider the semigroup $\hat{T}_{t}$ defined in Section 2.6 and denote by $\Omega=\{x(t) \in X: t>0\}$ the path space of the corresponding diffusion. For that path space we shall show that the criterion at the end of Section 2.6 holds. This will complete the proof of theorem for the above group. We shall adopt the following notation

$$
r_{s}=\gamma_{1} \gamma_{2} \cdots \gamma_{s} \in R, \quad s \geq 1, \gamma_{j} \in R, j=1,2, \ldots, s
$$

where we use group multiplication in both (5.2) and (5.3), but where, unless $K=\{e\}$, the $\gamma_{1}, \gamma_{2}, \cdots \in R$ are not independent random variables. As we pointed out in Section 2.8 however if we fix $k^{\infty}=\left(k_{j}\right)_{j=1}^{\infty} \in$ $K^{\infty}$ (some path in $K$ ) and condition with respect to that path the variables $\gamma_{1}, \gamma_{2}, \ldots$ become independent with uniformly Gaussian densities on $R$ (cf. sections 3.2 and 3.5). It follows that under that condition $r_{1}, r_{2}, \ldots$ becomes a time inhomogeneous random walk on $R$.

The following events $A_{1}, \ldots, B_{1}, \cdots \subset \Omega$ will now be considered

$$
A_{s}=\left[\left|\gamma_{j}\right|_{G} \leq C \log s ; j=1,2, \ldots, s\right], \quad s=1,2, \ldots,
$$


where $C>0$ will be chosen appropiately at the end. By the above (uniform in $j$ ) Gaussian estimate on the variables $\gamma_{j}$ we have the following estimate on the conditional expectations (uniformly in $k^{\infty}$ )

$$
\mathbb{P}\left[\sim A_{s} / / k^{\infty}\right] \leq \exp \left(-c \log ^{2} s\right), \quad s=1,2, \ldots,
$$

where $\sim$ stands as usual, for complement. Therefore we have

$$
\mathbb{P}\left[\sim A_{s}\right] \leq \exp \left(-c \log ^{2} s\right)
$$

Observe that with the notations of (5.1) on the event $A_{s}$ we have (cf. $3.1 .4)$

$$
\left|q_{j}\right|_{Q} \leq c \log s,\left|n_{j}\right|_{N} \leq C \exp (c \log s)=C s^{C}, \quad \gamma_{j}=\left(n_{j}, q_{j}\right)
$$

for $j=1,2, \ldots, s$. Let now

$$
B_{s}=\left[L_{k}\left(q_{1} \cdots q_{j}\right) \leq C ; j=1, \ldots, s ; k=1, \ldots, n\right] .
$$

Here $C>0$ and $L_{1}, \ldots, L_{n}$ are the real roots attached to the semidirect product $N \wedge Q$ as defined in Section 1.6. The basic fact that follows from Section D in the appendix ( $c f$. D.2) is that

$$
\mathbb{P}\left[B_{s}\right] \geq c s^{-C}, \quad s=1,2, \ldots,
$$

for appropiate constants $C, c>0$. When the operator $\Delta$ is elliptic the analogous even stronger statement (with the continuous time parameter) is a consequence of $\mathrm{A}(1)$ which was proved with considerably less cost in Section A of the appendix.

Let us now define the set

$$
X_{s}=\left\{x=(n, q, k) \in X:|n|_{N} \leq C s^{C},|q|_{Q} \leq C s^{C}\right\}
$$

It is then clear from the above and from Section 1.6 that

$$
A_{s} \cap B_{s}=\Omega_{s} \subset\left[x(s) \in X_{s}\right]
$$

and therefore that $\mathbb{P}\left[x(s) \in X_{s}\right] \geq c s^{C}$.

On the other hand we clearly have

$$
d^{r} r \otimes d k \text {-measure }\left[X_{s}\right] \leq C s^{C}
$$


and therefore our criterion of Section 2.6 is verified and we are done.

The following remark is worth making. We have used here the fact that in our criterion we can use indiscriminately either the $d^{r} r \otimes d k$ or the $d^{\ell} r \otimes d k$ measure to measure the set $X_{s}$. There is a very simply way to avoid this. Towards that let us define

$$
C_{s}=[|x(s)| \leq C \log s]
$$

then clearly by the Gaussian estimate on the Heat kernel on $G$

$$
\mathbb{P}\left[\sim C_{s}\right] \leq C \exp \left(-c \log ^{2} s\right)
$$

and

$$
\left|m_{G}(x)^{ \pm 1}\right|,\left|m_{R}(x)^{ \pm 1}\right| \leq C s^{C}, \quad|x| \leq C \log s .
$$

This means that if we replace $X_{s}$ by $X_{s} \cap\left[x \in X ;|x|_{G} \leq C \log s\right]$ and $\Omega_{s}$ by $A_{s} \cap B_{s} \cap C_{s}$ we obtain a new $X_{s}$ that satisfy the criterion as before and that furthermore on these new sets $X_{s}$ the two measures $d^{r} r \otimes d k$ and $d^{\ell} r \otimes d k$ are equivalent up to a constant that grows at most polynomically in $s$. Because of this it follows that it does not matter which of the two measures we consider.

\subsection{General NB-groups.}

From the above special case I shall deduce here the lower estimate (0.2) of Theorem A for a general group. Let $G$ be an arbitrary real NBLie group and let $\tilde{G} \rightarrow G$ be the simply connected cover of $G$. It clearly is enough to prove the NB-theorem for $\tilde{G}$ for then by the standard local Harnack principle the theorem also holds for $G$. We have that $\tilde{G}=Q \wedge S$ where $Q$ is the radical (simply connected) and $S$ is a simply connected semisimple group. By considering $S=N A \tilde{K}$ the Iwasawa decomposition of $S$ we can write then $\tilde{G}=R \tilde{K}$ with $R=Q N A$ but where $\tilde{K}$ is not necessarily compact. By general considerations however (cf. [15]) there exists $Z \subset \tilde{K}$ a discrete central (in $\tilde{G}$ ) subgroup such that $K=\tilde{K} / Z$ is compact. We have $\tilde{G} / Z=R K$ and therefore the lower estimate in (0.2) holds for the group $\tilde{G} / Z$. We shall now show how one deduces from this the same lower estimate for $\tilde{G}$ and therefore also for $G$.

We start with the following definition. Let $G$ be a compactly generated locally compact group and let $H \subset G$ be a closed compactly 
generated subgroup (e.g. $G$ a real Lie group and $H=\Gamma$ some discrete subgroup). As we already pointed out in Section 3.1 for any $h \in H$ the two distances $|h|_{H}$ and $|h|_{G}$ are not in general equivalent (we use here the more general notion of $|\cdot|_{H}$ valid for non connected groups, $c f$. [1]). We shall say that $H$ is a O-distortion subgroup if for all $\Omega \subset H$ neighbourhood of $e \in H$, there exists $C>0$ such that

$$
C^{-1}|h|_{G} \leq|h|_{H} \leq C|h|_{G}, \quad h \in H \backslash \Omega .
$$

The important thing to observe is that the central subgroup $Z \subset S \subset$ $\tilde{G}=Q \curlywedge S$ considered above is a O-distortion subgroup of $\tilde{G}$. This fact is easy to prove and the details were outlined in [15]. The fact that in the lower estimate $(0.2)$ we can pass from $\tilde{G} / Z$ to $\tilde{G}$ is therefore a consequence of the following

Lemma. Let $\pi: \check{G} \rightarrow G$ be a covering map and let $\Gamma=\operatorname{Ker} \pi \subset \check{G}$ be a O-distortion finitely generated subgroup. Let us further consider $\Delta_{0}=-\sum X_{j}^{2}$ some driftless sublaplacian on $G$ which can be identified with a sublaplacian on $\check{G}$. Let $\phi_{t}(g), \check{\phi}_{t}(g)$ be the corresponding Heat diffusion kernels and let $\lambda=\tilde{\lambda}$ be the corresponding spectral gap as in Section 0 (cf. (4.7.2)). We have

$$
\phi_{t}(e)=\sum_{\gamma \in \operatorname{Ker} \pi} \check{\phi}_{t}(\gamma), \quad t \geq 0
$$

and there exists $C>0$ such that

$$
\phi_{t}(e) \leq C \check{\phi}_{t}^{1 / 2}(e) e^{-\lambda t / 2} t^{C}, \quad t \geq 1 .
$$

The reader could observe that $\Gamma$ is automatically finitely generated but this point is here irrelevant.

Proof. $\Gamma \subset \check{G}$ is a central subgroup it follows therefore that $\check{\phi}_{t}(\gamma)$ is a positive definite function on $\Gamma$ and therefore $\check{\phi}_{t}(\gamma) \leq \check{\phi}_{t}(e), \gamma \in \Gamma$. We clearly also have $m_{G}(\gamma)=1, \gamma \in \Gamma$. By $([1$, Chapter 9, Section 1]) and the O-distortion property we also have

$$
\check{\phi}_{t}(\gamma) \leq C e^{-\lambda t} \exp \left(-\frac{|\gamma|^{2}}{c t}\right), \quad \gamma \in \Gamma, t \geq 1,
$$

for some $C>0$, and therefore also

$$
\check{\phi}_{t}(\gamma) \leq C \check{\phi}_{t}^{1 / 2}(e) e^{-\lambda t / 2} \exp \left(-\frac{|\gamma|^{2}}{c t}\right), \quad \gamma \in \Gamma, t \geq 1
$$


for a different $c, C>0$. If we apply the summation (5.6), which is trivial to prove ( $c f .(4.7 .1))$, our estimate (5.7) follows.

REMARK. An adaptation of the above method, with the use of the i'), ii') version of the criterion in Section 2.6, gives the proof of the lower estimate in Theorem $\left.\mathrm{A}_{1}\right)$. What changes is the geometry and the "section" that is used ( $c f .[13, \mathrm{II}])$.

If we use the global structure theorem for (not necessarily simply connected) NC-groups from [13, III] we can adapt the method of Section 5.1 to general NB-groups. The Section 5.2 becomes then redundant.

\section{Appendix.}

\section{Guide to the appendix.}

For the upper estimate of Theorem $A_{1}$ ) and for Theorem B one only needs Section B of this appendix. My advise to the reader in a first reading is to go straight for Section B and simply refer back for the notations.

Sections A.0 and A.1 suffice for the lower estimate of Theorem $\mathrm{A}_{2}$ ) in the case when $\Delta$ is an elliptic operator. In my mind this should be the next thing that the reader should study. To do this one should also study (or at least believe) Section C. Section C is elementary calculus but a certain amount of ingenuity is already needed. The estimate $\mathrm{A}(2)$ in Section A.3 is needed for the lower estimate of Theorem $A_{1}$ ). Had it not been for the non elliptic Laplacians $\Delta$ we would stop there and then. The discrete formulation and the discretisation presented in Section D and Section E are only needed to cope with this subelliptic (but not elliptic) situation, and Section F stands towards Section D what Section C stoud for Section A.1. More explicitely for the (non elliptic) lower estimate of Theorem $\mathrm{A}_{2}$ one needs the first half of Section F, Section E.2 and Section D.1 (i.e. $\mathrm{D}(2)$ for $p=+\infty)$. The property $\mathrm{D}(1)$ can be used as an alternative to $A(2)$ for the proof of the lower estimate of Theorems $\mathrm{A}_{1}$ ).

Both Section D and Section F are non trivial (in fact they are, technically, quite difficult) and they present an independent interest. I intend to come back in the future to the problems involved in sections $\mathrm{D}$ and $\mathrm{F}$ and examine them systematically for their own sake. The reader who is not particularly interested in these problems should 
simply not waste time and energy in these sections. Indeed an alternative approach (more sophisticated, but technically simpler) to the subelliptic Laplacians will be given in a second instalment of this work.

\section{A. The continuous time diffusion.}

\section{A.0. Statement of the results.}

We shall consider here the space $X=\mathbb{R}^{n} \times K$ where $K$ is a some compact $C^{\infty}$-manifold assigned with some smooth non vanishing measure $d k$. On $X$ we shall further consider $D$ some subelliptic formally self adjoint (with respect to $d x=d z \otimes d k$ where $d z$ is Lebesgue measure on $\mathbb{R}^{n}$ ) second order operator with constant term $D 1 \equiv 0$. $D$ will be assumed invariant under the left action of $\mathbb{R}^{n}$ (cf. Section 2.7). To simplify notations (and since this is the only case that we shall use) we shall further assume here that $K$ is some compact group and $d k$ is the Haar measure. The general case when $K$ is an arbitrary $C^{\infty}$-manifold can be treated with identical methods.

Let us denote by $e=(1,0, \ldots, 0) \in \mathbb{R}^{n}$ and by $C_{\alpha}=\left\{z \in \mathbb{R}^{n}\right.$ : $\langle z, e\rangle \geq|z| \cos \alpha\}(0<\alpha<\pi / 2)$ the corresponding conical region. Let us further denote by $C_{\lambda, \alpha}=C_{\alpha}-\lambda e(\lambda>0)$ the above conical region translated backwards so as to contain the origin $0 \in C_{\lambda, \alpha}$.

We shall now consider the continuous time diffusion on $X$

$$
\Omega=\left\{x(t)=(z(t), k(t)) \in X=\mathbb{R}^{n} \times K ; t>0\right\}
$$

controlled by the differential operator $D$. I shall denote as usual by $\mathbb{P}_{x}$ $(x \in X)$ the corresponding probability measure on $\Omega$ with $\mathbb{P}_{x}[x(0)=$ $x]=1$. We shall show that for any $0<\alpha<\pi / 2$ and $\lambda>0$ there exists $c>0$ such that

$$
\mathbb{P}_{0}\left[x(s) \in C_{\lambda, \alpha} \times K ; 0<s<t\right] \geq t^{-c}, \quad t>c,
$$

i.e. the diffusion stays in the conical region "polynomially long".

We shall also show that there exist $C>0$ such that

$$
\mathbb{P}_{0}[|x(s)| \leq M ; 0<s<t] \geq C^{-1} \exp \left(-C \frac{t}{M^{2}}\right), \quad t, M \geq 1,
$$

where for $x=(z, k) \in \mathbb{R}^{n} \times K$ we denote $|x|=|z|$. 
Let us observe straight away that $M=t^{1 / 3}$ in (A.2) gives

$$
\mathbb{P}_{0}\left[|x(s)| \leq t^{1 / 3} ; 0<s<t\right] \geq C^{-1} \exp \left(-C t^{1 / 3}\right), \quad t \geq 1
$$

\section{A.1. The differential operator $D$.}

In this section we shall consider the operators $-D=D_{R}+M+$ $P+D_{K}$ on $X=\mathbb{R}^{n} \times K$ as in sections 2.7 and A.0 and preserve all the notations introduced there.

Let $U(x) \in C^{\infty}(\Omega)\left(\Omega \subset \mathbb{R}^{n}\right.$, open $)$ and let $\psi_{j}(k) \in C^{\infty}(K)$, $(j=1, \ldots, n)$ be arbitrary. I shall denote by $F(x, k)=U\left(x_{1}+\right.$ $\left.\psi_{1}(k), \ldots, x_{n}+\psi_{n}(k)\right)$. We have then

$$
\begin{gathered}
D_{K} F=\sum_{j=1}^{n} D_{K}\left(\psi_{j}\right) \frac{\partial U}{\partial x_{j}}+\sum_{i, j=1}^{n}\left(\sum_{\alpha, \beta=1}^{s} \gamma_{\alpha, \beta}\left(X_{\alpha} \psi_{i}\right)\left(X_{\beta} \psi_{j}\right)\right) \frac{\partial^{2} U}{\partial x_{i} \partial x_{j}}, \\
M F=2 \sum_{i, j=1}^{n}\left(\sum_{\alpha=1}^{s} b_{\alpha, i} X_{\alpha} \psi_{j}\right) \frac{\partial^{2} U}{\partial x_{i} \partial x_{j}}, \\
P F=\sum_{j=1}^{n} \delta_{j} \frac{\partial U}{\partial x_{j}} .
\end{gathered}
$$

By (2.7.6) it then follows (this is standard Fredholm theory $c f$. [23], [24], [39] when $D$ is elliptic and the result easily generalizes to subelliptic operators) that for $j=1,2, \ldots, n$ we can choose $\psi_{j} \in C^{\infty}(K)$ so that

$$
D_{K}\left(\psi_{j}+c_{j}\right)+\delta_{j}=0, \quad\left(c_{1}, \ldots, c_{n}\right) \in \mathbb{R}^{n} .
$$

With that choice of the $\psi$ 's we have therefore $-D F=(L U)(x+\psi)$ where

$$
\begin{gathered}
L=\sum_{i, j=1}^{n} R_{i j}(k) \frac{\partial^{2}}{\partial x_{i} \partial x_{j}}, \\
R_{i, j}=a_{i, j}+\sum_{\alpha, \beta=1}^{s} \gamma_{\alpha, \beta} c_{\alpha, i} c_{\beta, j}+2 \sum_{\alpha=1}^{s} b_{\alpha, i} c_{\alpha, j}, \quad i, j=1, \ldots, n,
\end{gathered}
$$


where $c_{\alpha, i}=X_{\alpha} \psi_{i}(i=1, \ldots, n, \alpha=1, \ldots, s)$. Let us use standard matrix notations and set $A=\left(a_{i j}\right) \in M_{n \times n}, B=\left(b_{\alpha, i}\right) \in M_{s \times n}$, $\Gamma=\left(\gamma_{\alpha, \beta}\right) \in M_{s \times s}$. The characteristic form of $-D$ then is

$$
\mathbf{a}=\left(\begin{array}{cc}
A & B^{T} \\
B & \Gamma
\end{array}\right) \in M_{n+s, n+s}
$$

and the caracteristic form of $L$ is

$$
F(\mathbf{a} ; C)=A+C^{T} \Gamma C+B^{T} C+C^{T} B,
$$

where $C=\left(c_{\alpha, i}\right) \in M_{s \times n}$. (T stands for the matrix transposition operator).

The matrix $\mathbf{a}$ in (A.1.1) is non negative ( $c f$. Section 2.7). This implies that $F(\mathbf{a} ; C) \gg 0$ and therefore in particular if we assume that $\mathbf{a} \gg \varepsilon I$ for some $\varepsilon>0$ (this is the order relation of symmetric matrices) we also have $F \gg \varepsilon I$. The proof of these facts is elementary linear algebra. Indeed assume first that $A, \Gamma$ are the identity matrices then $\mathbf{a} \gg 0$ implies that $\Lambda^{T} \mathbf{a} \Lambda \geq 0\left(\Lambda^{T}=\left(\lambda^{T}, \mu^{T}\right), \lambda \in \mathbb{R}^{n}, \mu \in \mathbb{R}^{s}\right)$ i.e. $\lambda^{T} \lambda+\lambda^{T} B^{T} \mu+\mu^{T} B \lambda+\mu^{T} \mu \geq 0$, setting $\mu=-B \lambda$ we obtain the required result $\lambda^{T} \lambda-\lambda^{T} B^{T} B \lambda \geq 0$. In general, by standard perturbation, we can assume that $A, \Gamma$ are invertible. We set then $C=\Gamma^{-1 / 2} D$ and obtain

$$
F=A-B^{T} \Gamma^{-1} B+\left(D^{T}+B^{T} \Gamma^{-1 / 2}\right)\left(D+\Gamma^{-1 / 2} B\right) .
$$

This means that it suffices to show that $A-B^{T} \Gamma^{-1} B \gg 0$. Towards that by conjugating $\mathcal{L}^{T} \mathbf{a} \mathcal{L}$ with

$$
\mathcal{L}=\left(\begin{array}{cc}
A^{-1 / 2} & 0 \\
0 & \Gamma^{-1 / 2}
\end{array}\right)
$$

we can reduce the problem to the case where $A$ and $\Gamma$ are the identity matrices which is the special case that has just been treated.

In terms of our differential operators the above says that $L$ is a second order operator on $\mathbb{R}^{n}$ with positive characteristic and that $L$ is uniformly elliptic on $\mathbb{R}^{n}$ if $D$ is uniformly elliptic on $X$. It is an unfortunate fact that we cannot replace ellipticity by subellipticity in the above statement (example: $n=1, K=\mathbb{T}, D=\partial^{2} / \partial x^{2}+\partial^{2} / \partial \theta^{2} \pm$ $2 \cos \theta \partial^{2} / \partial x \partial \theta$, then $\partial^{2} \psi / \partial \theta^{2}=\mp \sin \theta$ and therefore $\partial \psi / \partial \theta= \pm \cos \theta$ $=c(\theta)$ and $\left.L=\sin ^{2} \theta \partial^{2} / \partial x^{2}\right)$. 
One important consequence of the above transformation where we set $U(x)=x_{i}(i=1, \ldots, n)$, the $n$ coordinate functions, is that the process

$$
y(t)=z(t)+\psi(k(t)) \in \mathbb{R}^{n}
$$

is a vector valued martingale. Equivalently this says that for any linear function $U(x)=\sum u_{j} x_{j}$ and any $C \in \mathbb{R}^{n}$ the function $F=U(x+$ $\psi(k)+C)$ is $D$-harmonic on $X$.

It is in general impossible to construct explicitely any other $D$ harmonic function on $X$. When $D$ is elliptic however it is very easy to give an explicit construction of an important family of $D$-subharmonic functions $F$ i.e. functions that satisfy

$$
D F(x) \leq 0 \text {. }
$$

More precisely let $\alpha>0$ be small and let $\tilde{C}=C_{\alpha} \times K$ be the conical region in $X$ as defined in Section A.0. Then a subharmonic function $F$ as in (A.1.3) can be constructed to have the following additional properties:

$$
F \in C^{k}(X) \text { for some (suitably high) } k>0, \quad F \geq 0,
$$

$$
F \equiv 0 \text { on } X \backslash \tilde{C}, \quad F \not \equiv 0 \text { on } \tilde{C},
$$

$$
\begin{aligned}
& F(x)=O\left(|x|^{A}\right) \text { for some } A>0, \\
& F\left(\mu e, e_{K}\right) \longrightarrow \infty \text { as } \mu \rightarrow \infty, \\
& e_{K}=\text { identity in } K .
\end{aligned}
$$

The construction of $F$ is easy. Indeed we start with $F_{r, k} \in C^{k+10}\left(\mathbb{R}^{n}\right)$ as in Section $\mathrm{C}$ and for an appropiate choice of $\nu, k$ we set $U(x)=F_{\nu, k}$ in our previous construction. It is clear then that if we choose appropiately the constants $C=\left(C_{1}, \ldots, C_{n}\right) \in \mathbb{R}^{n}$ the function

$$
F(x, k)=U\left(x_{1}+\psi_{1}+C_{1}, \ldots, x_{n}+\psi_{n}+C_{n}\right)
$$

has all the required properties. The role of the constants $C$ is simply to translate the value of the argument (on $\partial C_{\alpha} \times K$ ) outside $\partial C_{\alpha}$ where $U$ is $\equiv 0$. 


\section{A.2. The proof of (A.1).}

All our notations will be preserved. We shall first prove the following

Lemma. There exist $C, c>0$ such that

$$
\mathbb{P}_{e}\left[\max _{0<s<t}|z(s)| \geq C t\right] \leq e^{-c t}, \quad t \geq 1
$$

In fact for our purposes we only need the following weaker statement

$$
\mathbb{P}_{e}\left[\max _{0<s<t}|z(s)| \geq c t\right]=O\left(t^{-A}\right), \quad t \geq 1,
$$

for any $A>1$. The proof of the lemma is easy. Let us define $T_{0}=0<$ $T_{1}<\cdots$ a sequence of stopping times by

$$
T_{j+1}=\inf \left\{t>T_{j}:\left|z(t)-z\left(T_{j}\right)\right| \geq 1\right\}
$$

For every $j \geq 0, y_{j}(t)=y\left(t+T_{j}\right)-y\left(T_{j}\right),(t>0)$, with $y(t)$ as in Section A.1, is then a martingale and it is easy to verify that $S_{j}(t)$, the S-function of this martingale, satisfies ( $c f$. [27, Section 5])

$$
\mathbb{E}\left(\exp \left(\alpha S_{j}^{2}\left(t \wedge \xi_{j}\right)\right) / / \mathcal{T}_{T_{j}}\right) \leq C, \quad \xi_{j}=T_{j+1}-T_{j}, t>0, j \geq 0
$$

for $\alpha>0$ small enough. Since clearly by the stochastic integral representation of that martingale we have $c_{1} t \leq S_{j}^{2}(t) \leq c_{2} t\left(0<t<\xi_{j}\right.$, $j \geq 0$ and some $c_{1}, c_{2}>0$ ) it follows that there exists $c>0$ such that

$$
\mathbb{P}\left[T_{j+1}-T_{j}=\xi_{j}>\lambda / / \mathcal{T}_{T_{j}}\right]=O\left(e^{-c \lambda}\right), \quad \mathbb{E}\left(\xi_{j}\right) \geq c>0 .
$$

One can then use Bernstein's inequality for the sum of independent random variables ( $c f$. [22]) which works in this more general context and deduces that

$$
\mathbb{P}_{e}\left[\max _{0<s<t}|z(s)| \geq c n\right]=\mathbb{P}\left[\xi_{1}+\cdots+\xi_{n} \leq t\right], \quad n \sim t,
$$

has the correct bound. This proves the lemma. 
One can also prove (A.2.1) directly using the Doob maximal theorem on the martingale $y(s)(s \geq 0)$ constructed in Section A.1. This however requires the estimate $\|y(s)\|_{p}=O\left(s^{2 / 3}\right)$ (for $p$-large enough). This final estimate is a consequence of the Gaussian decay

$$
\mathbb{P}[|z(s)| \geq M]=O\left(\exp \left(-c \frac{M^{2}}{s}\right)\right)
$$

which although correct is not trivial to prove [19]. The subellipticity of $D$ is needed for that estimate to hold. In our context the above Gaussian estimate can also be picked up by the corresponding Gaussian estimate on the original group $G$ (cf. [1]).

Finally, for yet another approach to prove (A.2.1) one can use $S(t)$ the S-function of the martingale $y(t)$. Using the Itô (stochastic integral) approach of the construction of the diffusion $x(t)(t>0)$ one sees inmediately that $\|S(t)\|_{\infty}=O\left(t^{1 / 2}\right)$. The only complication here is of course the fact that $X$ is not $\mathbb{R}^{M}$ but a manifold and the construction has to be done in "patches", $c f$. [28]. The estimate (A.2.1) follows again by the standard martingale inequality $\|S\|_{p} \approx\|\max \|_{p}$ (cf. [27]). The advantage of this approach is that again no subellipticity is used.

Let now $\tilde{C}^{\prime}$ and $F(x), x \in X$, be as in A.1 and satisfy the conditions (A.1.4)-(A.1.5). We shall start diffusion at $O=\left(\lambda e, e_{K}\right) \in C \times K$ some large $\lambda>0$ and denote

$C_{R}=\tilde{C} \cap[a \leq|x| \leq R] \subset \mathbb{R}^{n}, \quad \tau=\tau_{R}=\inf \left\{t: x(t) \in \partial C_{R} \times K\right\}$

The standard submartingale property of the process $\{F(x(t)) ; t>0\}$ implies then that

$$
F(O) \leq \mathbb{E}\{F[x(\tau)]\} \leq C_{0}+\mathbb{P}[|x(\tau)|=R] R^{C}
$$

where the $C_{0}>0$ is independent of $R$ and comes from the fact that $x(t)$ could exist at some small $x(\tau)$ on which $F(x(\tau))>0$. If we choose $\lambda>0$ large enough however we are going to have $F(O)>2 C_{0}$ and therefore

$$
\mathbb{P}[|x(\tau)|=R] \geq c R^{-C}, \quad R \geq 1
$$

Our lemma on the other hand implies

$$
\mathbb{P}[\tau<R ;|x(\tau)|>c R]=O\left(R^{-A}\right)
$$


for all $A>0$. If we put (A.2.6) and (A.2.5) together we conclude that

$$
\mathbb{P}[\tau>R] \geq c R^{-C}, \quad R \geq 1 .
$$

This clearly implies (A.1).

\section{A.3. The proof of (A.2).}

The estimate (A.2) can easily be transformed to a standard " 1 rst eigenvalue" estimate. Indeed let us consider the operator $D$ on the M-ball of $X$

$$
X_{M}=\{x=(z, k):|z| \leq M\}
$$

with Dirichlet boundary conditions (i.e. we "kill" the diffusion at the boundary) and let $\lambda>0$ be the first eigenvalue and $0 \leq \psi \in C^{\infty}\left(X_{M}\right)$, $\|\psi\|_{2}=1$ the corresponding eigenfunction. Then clearly

$$
\left\|e^{-t D} 1\right\|_{2} \geq\left\langle 1, e^{-t D} \psi\right\rangle=e^{-t \lambda} \int_{X_{M}} \psi d x .
$$

Using standard methods we shall presently see that

$$
\int \psi d x \geq c>0, \quad \lambda \leq C M^{-2} .
$$

It follows thus that for each $t>0$ there exists some $x_{0} \in X_{M}$ such that

$$
\begin{aligned}
Q\left(x_{0}, t\right) & =\mathbb{P}_{x_{0}}[|x(s)| \leq M ; 0<s<t] \\
& \geq C \exp \left(-c \frac{t}{M^{2}}\right), \quad t>0 .
\end{aligned}
$$

By the left action of $\mathbb{R}^{n}$ on $X$ we can assume that $x_{0}=\left(0, k_{0}\right) \in K$. To show that we can assume that $x_{0}=0$ we can use the parabolic Harnack estimates that are verified by $Q(x, t)$ (these use the subellipticity of $D$ ). Otherwise (without the use of the above Harnack) we have automatically from (A.3.1)

$$
\sup _{x_{0} \in K} \mathbb{P}_{x_{0}}[|x(s)| \leq M ; 0<s<t] \geq C \exp \left(-c \frac{t}{M^{2}}\right), \quad M, t>0 .
$$

The estimate $\lambda \leq C M^{-2}$ in (A.3.2) is easy enough and is an immediate consequence of the fact that the function

$$
\varphi(z, k)=(M-|z|)^{+}, \quad(z, k) \in X
$$


appropiately smoothed for $|z|$ near 0 and $M$ satisfies

$$
\|\varphi\|_{2}^{2} \approx M^{n+2}, \quad(D \varphi, \varphi) \approx M^{n}
$$

The first estimate in (A.3.2) is a trifle more subtle (but also very standard). The subellipticity of $D$ implies (cf. [1]) that

$$
\|f\|_{p} \leq C\left[(D f, f)^{1 / 2}+\|f\|_{2}\right], \quad f \in C_{0}^{\infty}(X),
$$

for some $p>2$. The estimate (A.3.3) applied to $\psi$ implies (since $M \geq 1$ ) that $\|\psi\|_{p} \leq C\|\psi\|_{2}$ which by standard convexity gives the required estimate. A less sophisticated method to see that $\int \psi>C$ is to combine directly the fact $\|\psi\|_{2}=1$ with the Harnack estimate. Subellipticity is again essential for this approach (if $\int \psi \sim 0,\|\psi\|_{2}=1$ then there exists $x_{0} \in X_{M}$ such that $\psi\left(x_{0}\right) \gg 0$ also by standard elliptic estimates we may suppose that $\operatorname{dist}\left\{x_{0}, \partial X_{M}\right\} \geq c_{0}>0$. Harnack applies on $\psi$ and does the rest).

\section{A.4. Gaussian estimates for a Laplacian with a drift.}

The Gaussian estimate for the heat kernel of a Laplacian with a drift term $\Delta=-\sum X_{j}^{2}+X_{0}$ is not quite standard and we shall outline the proof here. The upper estimates are contained in [20] but here the proof does not need the rather difficult technology of [20] and this proof is already implicit in [19,I]. Indeed if $u(t, x)$ is a solution of $\partial / \partial t-\Delta$ then $v(t, x)=u\left(t, x e^{t X_{0}}\right)$ is a solution of the (time dependent) evolution equation

$$
\frac{\partial}{\partial t}-\sum\left(\operatorname{Ad}\left(e^{t X_{0}}\right) X_{j}\right)^{2}=0
$$

Let $\left\{T_{s, t}: 0<s<t\right\}$ be the corresponding time inhomogeneous semigroup [29] and let $\varphi \in C^{\infty}$ be such that $\left|\nabla_{r} \varphi\right| \leq 1$ where $\nabla_{r}$ denotes the gradient of some fixed left invariant Riemmanian structure on $G$. By the standard argument ( $c f .[19, \mathrm{I}])$ we then see that

$$
\left\|e^{\lambda \varphi} T_{t, s} e^{-\lambda \varphi}\right\|_{2 \rightarrow 2} \leq \exp \left(-c(t-s) \lambda^{2}\right)
$$

To give the proof of the upper Gaussian estimate of the corresponding Heat kernel (and of all its derivatives) we simply use the local Harnack principle just as in $[19, \mathrm{I} ; 1]$. One should simply observe that the 
canonical distance on $G$ induced by the fields $X_{1}, \ldots, X_{n}$ and the above Riemmanian distance are "equivalent" at infinity.

It is of independent interest to observe that the following maximal Gaussian estimate also holds (and we do not need subellipticity for this estimate)

$P_{t}(\gamma)=\mathbb{P}\left[\max _{0<s<t}|z(s)| \geq \gamma\right] \leq C \exp \left(-c \gamma^{2} / t\right), \quad 0<t<1, \gamma / t>1$

and that there here exists an alternative more direct proof of this fact. This proof relies on a standard Laplace transform argument ( $c f$. [21]) and the estimate $P_{t}(1) \leq c e^{-c / t}(t>0)$ which is equivalent to

$$
\mathbb{P}[T=\inf [s ;|z(s)| \geq 1]<t] \leq c e^{-c / t}, \quad t>0 .
$$

This last estimate is non trivial. The only way I know how to prove it is by considering in local coordinates the semimartingale expression of $t^{-1 / 2} z(s \wedge t \wedge T),(s>0)$ for fixed $t \leq 1$. It is easy to see then that the S-function of that semimartingale satisfies $\|S\|_{\infty} \leq C$. This implies (well known: we time change the martingale part of the semimartingale and make it brownian motion) that the maximal function

$$
M^{*}=t^{-1 / 2} \sup _{0<s<t}|z(s \wedge T)|
$$

satisfies $\left\|\exp \left(\alpha\left(M^{*}\right)^{2}\right)\right\|_{\infty} \leq C$ and our estimate (A.4.1) follows from the fact that on the set $[T<t]$ we have $M^{*}=t^{1 / 2}$.

The proof of the lower Gaussian estimate (unless the drift is of special form, $c f$. [19]) is as far as I can tell considerably more difficult to prove. The pivot of the proof is the estimate

$$
\mathbb{P}_{x}\left[d(z(t), y) \leq 10^{-10}\right] \geq C \exp \left(-\frac{c}{t}\right),
$$

for $0<t<1, x, y \in G, 10^{-10} \leq d(x, y) \leq 10^{10}$. This estimate for a Laplacian with a drift is essentially the Varadhan-Ventcel-Freidlin large deviations estimates for the Heat kernel ( $c f$. [30]). The details are rather formidable to write out. This has been done in [19, II] (esp. Section 4.3). In that reference the drift had a special form but the proof given there works for a general drift. From (A.4.2) the lower Gaussian estimate follows by standard methods (e.g. [19,II], Section 2.4). 


\section{B. The large deviation estimate.}

In this section we shall preserve all the notations of Section 4.4 and we shall prove the estimate (4.4.1). The proof is done in two steps. The first step consists in modifying

$$
\tilde{A}\left(L_{i}\right)=\inf _{0 \leq j \leq n} \exp \left(-d_{i} L_{i}\left(b_{j}\right)\right)
$$

where we set $b_{0}=0$ and in showing that

$$
\mathbb{E}\left(\tilde{A}_{n}\left(L_{1}\right) \cdots \tilde{A}_{n}\left(L_{p}\right)\right)=O\left(\exp \left(-c n^{1 / 3}\right)\right) .
$$

The second step consists in deducing (4.4.1) from (B.2).

To simplify notations I shall also assume throughout that $d_{i}=1$ $(i=1, \ldots, p)$. At any rate in both (4.4.1) and (B.1) we can also absorb the $d_{i}$ with the $L_{i}$ and consider $\tilde{L}_{i}=d_{i} L_{i}$ instead.

Proof of The SteP 1 . By the C-condition we can fix $\ell \geq 2,1 \leq i_{1}<$ $\cdots<i_{\ell} \leq p$ and $\alpha_{s}>0(1 \leq s \leq \ell)$ such that

$$
\alpha_{s} L_{i_{s}} \neq 0,1 \leq s \leq \ell, \quad \sum_{s=1}^{\ell} \alpha_{s} L_{i_{s}}=0 .
$$

It is then clear from the geometry of the situation that there exists $C>0$

$$
\left|L_{i_{1}}(x)\right| \leq C \sum_{s=1}^{\ell} L_{i_{s}}^{+}(x), \quad x \in V
$$

therefore since

$$
\tilde{A}_{n}\left(L_{1}\right) \cdots \tilde{A}_{n}\left(L_{p}\right) \leq \exp \left(-\sup _{1 \leq j \leq s} \sum_{s=1}^{\ell} L_{i_{s}}^{+}\left(b_{j}\right)\right)
$$

we conclude that (B.2) will follow as soon as we can prove that for any $a>0$ we have

$$
\mathbb{E}\left(\exp \left(-a \sup _{1 \leq j \leq n}\left|L_{i_{1}}\left(b_{j}\right)\right|\right)\right)=O\left(e^{-c n^{1 / 3}}\right),
$$

where $c>0$.

Observe now that $U_{k}=L_{i_{1}}\left(X_{k}\right) \in \mathbb{R},(k=1,2, \ldots)$ is a sequence of real random variables and that the density functions $\psi_{k}$ of these 
variables $\mathbb{P}\left[U_{k} \in d x\right]=\psi_{k}(x) d x$ clearly are Gs-functions on $\mathbb{R}$ (by Chapter 3) uniformly in $j=1,2, \ldots$ Our estimate (B.3) is thus a consequence of the following lemma

Lemma 1. Let $U_{1}, U_{2}, \ldots$ be a arbitrary sequence of independent random variables that satisfies the above condition. Let $S_{n}=U_{1}+\cdots+U_{n}$ and $V_{n}=\sup _{1 \leq j \leq n}\left|S_{j}\right|$. Then for every $a>0$ there exists $c>0$ such that

$$
\mathbb{E}\left(e^{-a V_{n}}\right)=O\left(e^{-c n^{1 / 3}}\right)
$$

Lemma 2. Let $U_{1}, U_{2}, \ldots$ be as in Lemma 1. Then there exists $\varepsilon>0$ and an integer $n_{0}$ such that

$$
\mathbb{P}\left[\left|U_{1}+U_{2}+\cdots+U_{n_{0} m^{2}}\right| \leq 2 m\right] \leq 1-\varepsilon, \quad m=1,2, \ldots
$$

Lemma 1 follows from Lemma 2. Indeed from Lemma 2 it is clear that

$$
\mathbb{P}\left[V_{p n_{0} m^{2}} \leq m\right] \leq(1-\varepsilon)^{p}, \quad m, p=1,2, \ldots,
$$

and therefore that

$$
\mathbb{P}\left[V_{n} \leq m\right] \leq C \exp \left(-c \frac{n}{m^{2}}\right), \quad n, m=1,2, \ldots,
$$

and (B.4) follows by integration.

Proof of Lemma 2. Let $\varphi_{k}(\xi)=\hat{\psi}_{k}(\xi), \xi \in \mathbb{R}$, denote the characteristic function of the variable $U_{k}, k=1,2, \ldots$ The uniform lower estimate of the Gs-condition $\psi_{k}(x) \geq C \exp \left(-c x^{2}\right)$ implies that $\psi_{k}(x)=$ $\alpha G(x)+(1-\alpha) \tilde{\psi}_{k}(x)$, where $G(x)$ is a Gaussian distribution and $\tilde{\psi}_{k}$ some other probability distribution and there exist thus $0<\alpha<1$, $c>0$ such that

$$
\left|\varphi_{k}(\xi)\right| \leq \alpha \exp \left(-c \xi^{2}\right)+1-\alpha, \quad k=1,2, \ldots
$$

Now again the uniform Gs-condition on the $\psi_{j}$ 's implies that $\varphi_{k} \in$ $C^{\infty}(\mathbb{R})$ uniformly in $k$ and therefore since

$$
\varphi_{k}(0)=1,\left|\varphi_{k}(\xi)\right|<1, \quad \xi \neq 0,
$$


it follows that there exists $c>0$ such that

$$
\left|\varphi_{k}(\xi)\right| \leq 1-c|\xi|^{2}, \quad|\xi|<c, k=1,2, \ldots
$$

Putting (B.5) and (B.6) together we conclude that there exists $c, \eta>0$ such that

$$
\left|\varphi_{k}(\xi)\right| \leq\left\{\begin{array}{ll}
1-\eta & \text { if }|\xi| \geq 1, \\
e^{-c|\xi|^{2}} & \text { if }|\xi| \leq 1,
\end{array} \quad k=1,2, \ldots\right.
$$

Let now

$$
0 \leq \chi, \hat{\chi} \in C_{0}(\mathbb{R}) \cap L^{1}(\mathbb{R}), \quad \hat{\chi}(\xi)=\int e^{i x \xi} \chi(x) d x .
$$

Then for any $m=1,2, \ldots, r>0$ we have

$$
\begin{aligned}
& \int \psi_{1} * \cdots * \psi_{m^{2}}(x) \chi\left(\frac{x}{r m}\right) d x \\
& =r m \int\left(\varphi_{1} \cdots \varphi_{m^{2}}\right)(\xi) \hat{\chi}(r m \xi) d \xi \\
& \leq r m \int e^{-c m^{2} \xi^{2}} \hat{\chi}(r m \xi) d \xi+(1-\eta)^{m^{2}} \chi(0) \\
& \leq \int e^{-c \xi^{2} / r} \hat{\chi}(\xi) d \xi+(1-\eta)^{m^{2}} \chi(0) \\
& \leq c \sqrt{r} \hat{\chi}(0)+(1-\eta)^{m^{2}} \chi(0) \leq 1-\delta,
\end{aligned}
$$

where the last estimate holds if $r$ is small enough and $m$ large enough. For an appropiate choice of $\chi$ we have $\chi(x) \geq 1,|x| \leq 1$ and thus (B.7) gives

$$
\mathbb{P}\left[\left|U_{1}+\cdots+U_{m^{2}}\right| \leq r m\right] \leq 1-\delta
$$

and Lemma 2 follows.

Proof of Step 2. Let us fix $N \geq 1$ (to be chosen later) and denote $I_{\alpha}=\{\alpha N+1, \alpha N+2, \ldots,(\alpha+1) N\} \subset \mathbb{N}, \alpha=0,1,2, \ldots$ Let $Y_{\alpha}=$ $\inf _{j \in I_{\alpha}}\left|X_{j}\right|$ and let $j_{\alpha} \in I_{\alpha}$ be the first integer $j \in I_{\alpha}$ for which $\left|X_{j}\right|=$ $Y_{\alpha}$. Let us further define

$$
\begin{gathered}
B_{n}\left(L_{i}\right)=\inf _{\alpha ; \alpha N \leq n+N} \exp \left(c Y_{\alpha}^{2}-L_{i}\left(b_{j_{\alpha}}\right)\right), \\
C_{n}\left(L_{i}\right)=\inf _{\alpha ; \alpha N \leq n+N} \exp \left(-L_{i}\left(b_{j_{\alpha}}\right)\right),
\end{gathered}
$$


892 N. Th. Varopoulos

$$
\zeta_{n}=\sup _{\alpha ; \alpha N \leq n+3 N}\left(\sum_{k \in I_{\alpha}}\left|X_{k}\right|\right), \quad \xi_{n}=\sup _{\alpha ; \alpha N \leq n+3 N}\left(Y_{\alpha}\right) .
$$

It is then clear that

$$
\begin{gathered}
A_{n+N}\left(L_{i}\right) \leq B_{n}\left(L_{i}\right) \leq C_{n}\left(L_{i}\right) e^{c \xi_{n}^{2}} \\
C_{n}\left(L_{i}\right) \leq \tilde{A}_{n}\left(L_{i}\right) e^{C \zeta_{n}}
\end{gathered}
$$

provided that $C>0$ is large enough.

By the independence and the Gaussian decay of the variables involved, we see on the other hand that

$$
\begin{gathered}
\mathbb{P}\left[\sum_{k \in I_{\alpha}}\left|X_{k}\right| \geq \lambda\right] \leq C \exp \left(-c \lambda^{2}\right), \quad \alpha=1,2, \ldots, \\
\mathbb{P}\left[\zeta_{n} \geq \lambda\right] \leq C n \exp \left(-c \lambda^{2}\right),
\end{gathered}
$$

where $C, c$ are independent of $n, \alpha=1,2, \ldots$, (but may depend on $N$ ) and that

$$
\begin{gathered}
\mathbb{P}\left[Y_{\alpha} \geq \lambda\right] \leq C_{0} \exp \left(-c_{0} N \lambda^{2}\right), \quad \alpha=1,2, \ldots, \\
\mathbb{P}\left[\xi_{n} \geq \lambda\right] \leq C_{0} n \exp \left(-c_{0} N \lambda^{2}\right),
\end{gathered}
$$

where $C_{0}, c_{0}$ are independent of $n, \alpha, N=1,2, \ldots$ It follows in particular that

$$
\left\|e^{c \zeta_{n}}\right\|_{p}=O\left(n^{1 / p}\right), \quad 1 \leq p \leq+\infty
$$

and that for every given $k>0$ and $1 \leq p<+\infty$ there exists an $N \geq 1$ large enough for which

$$
\left\|e^{k \xi_{n}^{2}}\right\|_{p}=O\left(n^{1 / p}\right)
$$

The proof of the step 2 is then a consequence of (B.2), (B.8), (B.9), (B.10), (B.11) and a simple use of Hölder's inequality. 


\section{The conical function and the Hessian.}

Let $e=(1,0, \ldots, 0) \in \mathbb{R}^{n}$ and let $r=|x|$, let $\varphi_{0}=\theta$ be the latitude with respect to the north pole $e$ and $\left(\varphi_{1}, \ldots, \varphi_{n-2}\right)$ be an appropiate set of local coordinates on $S_{n-2}$ (the $n-2$ sphere), so that $\left(r, \theta, \varphi_{1}, \ldots, \varphi_{n-2}\right)$ are a set of "polar" coordinates on $\mathbb{R}^{n}$.

We shall now fix some small $0<\theta_{0}$ and define a function $F=F_{\nu, k}$ on $\mathbb{R}^{n}$. First of all $F \equiv 0$ if $\langle x, e\rangle \leq|x| \cos \theta_{0}$ i.e. $F \equiv 0$ outside the region $C_{\alpha}$ (cf. Section A.0) with $\alpha=\theta_{0}$. Next we require that $F(x)=r^{\nu} u(\theta)$ for $x \neq 0$ for some large $\nu=1,2, \ldots$ and $0 \leq u(\theta) \in$ $C^{k}$. The function $u(\theta), 0 \leq \theta \leq \pi$ will have the following properties $u(\theta)>0$ for $-\theta_{0}<\theta<\theta_{0} ; u(\theta) \equiv 1$ for $-\varepsilon<\theta<\varepsilon$ (for some small $\varepsilon$ ) and $u(\theta)=\left(|\theta|-\left|\theta_{0}\right|\right)^{k}$ for ||$\theta|-| \theta_{0}||$ small where $k=2,4, \ldots$ is an appropiate even integer. In this section we shall analyze the Hessian $H_{\nu, k}=\operatorname{Hess}(F)=\left(h_{i, j}\right)$.

Quite generally let us denote by $\mathcal{S}$ the set of symmetric real $n \times n$ matrices and by $\mathcal{P} \subset \mathcal{S}$ the cone of non negative matrices. Let $\ell=$ $\left(\ell_{1}, \ldots, \ell_{n}\right) \in \mathbb{R}^{n}$, we shall denote by $\ell \otimes \ell=\left(\ell_{i} \ell_{j}\right) \in \mathcal{P}$. It is clear that any $s \in \mathcal{S}$ can be written

$$
s=\sum_{j=1}^{k} \lambda_{j}^{+} \ell_{j}^{+} \otimes \ell_{j}^{+}-\sum_{i=k+1}^{n} \lambda_{i}^{-} \ell_{i}^{-} \otimes \ell_{i}^{-},
$$

where $\lambda_{j}^{ \pm} \geq 0$ are the characteristic roots and $\ell_{j}^{ \pm}(j=1,2, \ldots)$ are the corresponding orthonormal set of eigenvectors. We shall finally define the scalar product in $\mathcal{S}$

$$
\left\langle S^{(1)}, S^{(2)}\right\rangle=\sum_{i, j} S_{i j}^{(1)} S_{i j}^{(2)}, \quad S^{(k)}=\left(S_{i j}^{(k)}\right) \in \mathcal{S} .
$$

The following two notations will be needed

$$
\begin{gathered}
\mathcal{P}_{a}=\left\{p=\left(p_{i j}\right) \in \mathcal{P}: a \sum\left|\mu_{j}\right|^{2} \geq \sum p_{i j} \mu_{i} \mu_{j} \geq a^{-1} \sum\left|\mu_{j}\right|^{2}\right. \\
\left.\qquad \text { for all }\left(\mu_{1}, \ldots, \mu_{n}\right) \in \mathbb{R}^{n}\right\}, \quad a \geq 1, \\
\mathcal{S}_{a}=\left\{s \in \mathcal{S}:\langle p, s\rangle \geq 0 \text { for all } p \in \mathcal{P}_{a^{1 / 2}}\right\}, \quad a \geq 1 .
\end{gathered}
$$

The connection between the above two definitions is described in the following elementary. 
Lemma. Let $s \in \mathcal{S}$ and $a \geq 1$. Then $s \in \mathcal{S}_{a}$ if and only if we can write $s$ in (C.1) with

$$
\sum \lambda_{j}^{+} \geq a\left(\sum \lambda_{j}^{-}\right)
$$

Proof. Indeed $p \in \mathcal{P}_{a^{1 / 2}}$ if and only if in (C.1) it can be written

$$
p=\sum \mu_{j} \pi_{j} \otimes \pi_{j}
$$

with $a^{-1 / 2} \leq \mu_{j} \leq a^{1 / 2}$ and $\pi_{j} \in \mathbb{R}^{n}$ some orthonormal basis. If we bare in mind that $\langle\ell \otimes \ell, \pi \otimes \pi\rangle=\langle\ell, \pi\rangle^{2}$ for the standard scalar product on $\mathbb{R}^{n}$ we see that with $s$ as in (C.1) and $p$ as in (C.3) we have

$$
\langle s, p\rangle=\sum_{j, \alpha} \lambda_{j}^{+} \mu_{\alpha}\left\langle\ell_{j}^{+}, \pi_{\alpha}\right\rangle^{2}-\sum_{j, \alpha} \lambda_{j}^{-} \mu_{\alpha}\left\langle\ell_{j}^{-}, \pi_{\alpha}\right\rangle^{2} .
$$

We clearly also have

$$
\sum_{\alpha}\left\langle\ell, \pi_{\alpha}\right\rangle^{2}=\|\ell\|_{2}^{2}, \quad \ell \in \mathbb{R}^{n}
$$

From (C.4) and (C.5) it follows that $\langle s, p\rangle \geq 0$ if (C.2) is verified. This gives the first half of the lemma. To see the opposite direction for $s \in \mathcal{S}$ as in (C.1) it suffices to test the condition $\langle s, p\rangle \geq 0$ on the matrix

$$
p=\sum_{j} a^{-1 / 2} \ell_{j}^{+} \otimes \ell_{j}^{+}+\sum_{j} a^{1 / 2} \ell_{j}^{-} \otimes \ell_{j}^{-} \in \mathcal{P}_{a^{1 / 2}} .
$$

The significance for us of the above notions lies in the following

Lemma. Let $a \geq 1$ be given. Then there exists $k_{0}=k_{0}(a)$ such that for all $k \geq k_{0}$ there exists $u(\theta)$, satisfying the conditions of the definition of $F_{\nu, k}$, and $\nu_{0}, \nu_{0}(a, u) \geq 3$ such that

$$
H_{\nu, k}=\operatorname{Hess}\left(r^{\nu} u(\theta)\right) \in \mathcal{S}_{a}, \quad \nu \geq \nu_{0},
$$

at every point of $\mathbb{R}^{n}$.

The first step is to observe that we have

$$
\nabla=\left(\frac{\partial}{\partial x_{1}}, \ldots, \frac{\partial}{\partial x_{n}}\right)=A\left(\frac{\partial}{\partial r}, \frac{1}{r} \frac{\partial}{\partial \varphi_{0}}, \ldots, \frac{1}{r} \frac{\partial}{\partial \varphi_{n-2}}\right)
$$


valid for $r>0$ and $\left|\theta-\theta_{0}\right| \leq\left|\theta_{0}\right| / 2$ and $\left(\varphi_{1}, \ldots, \varphi_{n-2}\right)$ in some appropiate patch of local coordinates of $S_{n-2}$. The matrix $A=\left(a_{i j}(\varphi)\right)$ has $C^{\infty}$ coefficients that only depend on $\left(\varphi_{0}, \ldots, \varphi_{n-2}\right)$ and is independent of $r$. For every $p \in \mathcal{P}$ and the corresponding differential operator $P$ we have therefore

$$
\begin{aligned}
P F= & \left\langle p, H_{\nu, k}\right\rangle \\
= & \sum p_{i j} \frac{\partial^{2} F}{\partial x_{i} \partial x_{j}} \\
= & s \frac{\partial^{2} F}{\partial r^{2}}+2 \frac{1}{r} \sum_{i=0}^{n-2} s_{i} \frac{\partial^{2} F}{\partial r \partial \varphi_{i}}+\frac{1}{r^{2}} \sum_{i, j=0}^{n-2} s_{i, j} \frac{\partial^{2} F}{\partial \varphi_{i} \partial \varphi_{j}}+\alpha \frac{1}{r} \frac{\partial F}{\partial r} \\
& +\frac{1}{r^{2}} \sum_{i=0}^{n-2} \beta \frac{\partial F}{\partial \varphi_{i}},
\end{aligned}
$$

where the coefficients are $C^{\infty}$ and where the matrix $\left(s_{i j} ; i, j=-1,0\right.$, $1, \ldots, n-2) \gg 0$ is positive definite (with $s_{-1,-1}=s, s_{-1, i}=s_{i}$ ). For $F=r^{\nu} u$ it follows that

$$
\begin{aligned}
r^{2-\nu} P F & =\nu(\nu-1) s u+2 \nu s_{0} u^{\prime}+s_{0,0} u^{\prime \prime}+\nu \alpha u+\beta_{0} u^{\prime} \\
& =\nu^{2}(s+O(1 / \nu)) u+2 \nu\left(s_{0}+O(1 / \nu)\right) u^{\prime}+s_{0,0} u^{\prime \prime} .
\end{aligned}
$$

Therefore for our special choice of $u(\theta)$ and $\theta$ close to $\theta_{0}\left(|\theta|<\left|\theta_{0}\right|\right)$ we have

$$
\begin{aligned}
r^{2-\nu} P F= & \nu^{2}\left(\theta-\theta_{0}\right)^{k}(s+O(1 / \nu)) \\
& +2 k \nu\left(\theta-\theta_{0}\right)^{k-1}\left(s_{0}+O(1 / \nu)\right) \\
& +k(k-1)\left(\theta-\theta_{0}\right)^{k-2} s_{0,0} .
\end{aligned}
$$

Given $a \geq 1$ it follows that the discriminant in (C.7)

$$
D=k^{2}\left(s_{0}+O(1 / \nu)\right)^{2}-k(k-1) s_{0,0}(s+O(1 / \nu))
$$

is strictly negative for all

$$
\left|\theta-\theta_{0}\right| \leq \varepsilon_{0}, \quad \nu \geq \nu_{0}, \quad k \geq k_{0}, \quad p \in \mathcal{P}_{a^{1 / 2}}
$$

where $\nu_{0}=\nu_{0}(a), k=k_{0}(a)$ only depend on $a$.

Let us fix some $k \geq k_{0}$ and some $u(\theta)$ that satisfies the conditions of the definition of $F$ (for that $k$ ). Once $u$ has been fixed, it follows 
from (C.6) that there exists $\nu_{1}=\nu_{1}(u) \geq \nu_{0}$ (that depends on $u$ ) such that $P F \geq 0$ for all $\left|\theta-\theta_{0}\right| \geq \varepsilon_{0}$ and $\nu \geq \nu_{1}$. This completes the proof of the lemma.

\section{The random walk and the martingale.}

\section{D.0. Statement of the results.}

Let $S_{j}=X_{1}+\cdots+X_{j} \in \mathbb{R}^{n}$ denote an $n$-dimensional random walk where the variables $X_{j}$ are independent and centered $\left(\mathbb{E}\left(X_{j}\right)=0\right.$, $j=1, \ldots)$, but not necessarily identically distribuited, and where there exists $a \geq 1$ such that each covariance matrix satisfies

$$
\left\{\mathbb{E}\left(X_{j}^{\alpha} X_{j}^{\beta}\right)\right\}_{\alpha, \beta=1}^{n} \in \mathcal{P}_{a}, \quad j \geq 1
$$

(here we use the notations of Section $\mathrm{C}$ and $X_{j}=\left(X_{j}^{1}, \ldots, X_{j}^{n}\right)$ are the coordinates). We shall also assume that for some $2 \leq p \leq+\infty$ we have

$$
\left\|X_{j}\right\|_{p} \leq C, \quad j=1,2, \ldots
$$

We shall generalize the above setup and consider a vector valued martingale

$$
f_{j}=d_{1}+\cdots+d_{j} \in \mathbb{R}^{n}, \quad j=1, \ldots, f_{0}=0 .
$$

The conditions we shall impose on the martingale differences will be a natural generalization of (D.0.1) and (D.0.2)

$$
\mathbb{E}\left(d_{j}^{\alpha} d_{j}^{\beta} / / \mathcal{T}_{j-1}\right) \in \mathcal{P}_{a}, \quad \mathbb{E}\left(\left|d_{j}\right|^{p} / / \mathcal{T}_{j-1}\right) \leq C, \quad j=1, \ldots,
$$

where $\mathcal{T}_{1} \subset \mathcal{T}_{2} \subset \cdots$ are the $\sigma$-fields of the martingale. Let $\left(f_{j} ; j \geq 0\right)$ be a martingale as above we shall then show that if $p>2$ there exists $C, c>0$ such that

$$
\mathbb{P}\left[\sup _{1 \leq j \leq n}\left|f_{j}\right| \leq M\right] \geq c \exp \left(-C \frac{n}{M^{2}}\right), \quad n, M \geq C
$$

We shall also show that (if $p \geq p_{0}$ large enough) for any $0<\alpha<1$ and $\lambda>0$ large enough there exists $\xi>0$ such that

$$
\mathbb{P}\left[f_{j} \in C_{\lambda, \alpha} ; 1 \leq j \leq m\right] \geq m^{-\xi}, \quad m \geq \xi,
$$


where $C_{\lambda, \alpha}$ is as in Section A.0.

Both the above estimates are very easy to prove when $n=$ dimension $=1$. For $n=1,(\mathrm{D} .2)$ is well known. We can take $p=1+\varepsilon>$ 1 arbitrary then, and we do not need the extra covariance condition. To see this we shall consider $u_{j}=F\left(f_{j}\right)$ where $F(x) \equiv 0, x \leq-1$, and $F(x)=x+1, x \geq-1$ which is a submartingale and then apply the "optional stopping time theorem" on $f_{j \wedge T}$ where $T=\inf \left\{j: f_{j} \leq-1\right\}$.

The proof of D.1 for $n=1$ is not very much harder. We set $T=\inf \left\{j:\left|f_{j}\right| \leq M\right\}$ and compose $f_{j}^{*}=f_{j \wedge T}$ with the function $F_{M}(x)=F(x / M)$ where $F \in C^{\infty}, 0 \leq F \leq 1, F(x) \equiv 0$ for $|x| \geq 1$, $F(x) \equiv 1$ for $|x| \leq 1 / 2, F(x)>0$ for $|x|<1$ and $F(x)=C(1-|x|)^{10}$ for $1-|x| \leq 1 / 10$. Using the Taylor series of $F$, it is easy to verify that ( $c f$. Section D.2 for details; in fact this verification is entirely trivial if $\left.\left|d_{k}\right| \leq 1\right)$

$$
\mathbb{E}\left[F_{M}\left(f_{n}^{*}\right) / / \mathcal{T}_{n-1}\right] \geq e^{-c M^{-2}} F_{M}\left(f_{n-1}^{*}\right), \quad n \geq 1 .
$$

If we iterate this for $n, n-1, \ldots$ we see that (D.1) follows at once.

Both (D.1) and (D.2) are false in higher dimensions without the covariance condition in (D.0.1). It is clear why (D.2) brakes down, it suffices in the random walk $S_{j}=X_{1}+\cdots+X_{j}$ to consider "singular" variables $X_{j} \in$ hyperplane perpendicular to the axis of $C_{\alpha}$. To see why (D.1) brake down when $n=2$ we start with $r_{j}=\left(r_{j}, 0\right) \in \mathbb{R}^{2}$, where $r_{j}= \pm 1$ are Rademacher variables, and consider $T_{j} \in S O_{2}$ so that the last vector $\left(\in \mathbb{R}^{2}\right)$ in the following summation

$$
f_{j}=r_{1}+T_{1}\left(r_{2}\right)+T_{2}\left(r_{3}\right)+\cdots+T_{j-1}\left(r_{j}\right)
$$

is orthogonal in $\mathbb{R}^{2}$ to the sum of the first $j-1$ terms. The rotations $T_{j}$ can clearly be made $\mathcal{T}_{j-1}$-measurable. We obtain thus a martingale transform (cf. [27]) that satisfies $\left|f_{j}\right| \equiv \sqrt{j}$.

REMARK. It is clear that the first condition (D.0.1) is equivalent to

$$
a \sum\left|\lambda_{\alpha}\right|^{2} \geq \mathbb{E}\left(\left|\sum_{\alpha=1}^{n} \lambda_{\alpha} d_{j}^{\alpha}\right|^{2} / / \mathcal{T}_{j-1}\right) \geq a^{-1} \sum\left|\lambda_{\alpha}\right|^{2} .
$$

Therefore when the $d_{j}$ 's admit conditional densities

$$
\mathbb{P}\left[d_{j} \in d y / / \mathcal{T}_{j-1}\right]=d \mu_{j, \omega}(y), \quad j=1,2, \ldots,
$$

the condition (D.0.1) is equivalent to

$$
\left(\int y_{\alpha} y_{\beta} d \mu_{j, \omega}(y)\right)_{\alpha, \beta=1}^{n} \in \mathcal{P}_{a}, \quad j=1,2, \ldots
$$




\section{D.1. A general notion of subharmonicity, the Taylor series and the proof of (D.2).}

We shall consider

$$
\mu_{x}^{(\alpha)} \in \mathbb{P}\left(\mathbb{R}^{n}\right), x \in \mathbb{R}^{n}, \alpha \in A=\text { some index set },
$$

a family of centered (i.e. $\int_{\mathbb{R}^{n}} y_{i} d \mu_{x}^{(\alpha)}(y)=0, x \in \mathbb{R}^{n}, \alpha \in A, i=$ $1, \ldots, n)$ probability measures and $F \geq 0$ some upper semicontinuous function on $\mathbb{R}^{n}$. Let further $\mathcal{R} \subset \mathbb{R}^{n}$ be some open domain. We shall then say that $F$ is subharmonic in $\mathcal{R}$ with respect to the above family if

$$
F(x) \leq F * \mu_{x}^{\alpha}(x), \quad x \in \mathcal{R}, \alpha \in A .
$$

For simplicity in what follow we shall drop the index $\alpha \in A$ and consider only the case when $A$ reduces to the one point set.

The interest for us of the above definition lies in the following considerations. We shall consider the family of measures (D.0.3). These measures in our applications will be given by $d \mu_{x}(y)=p(y, x) d y$ where the $p$ 's are as in Section E. We shall furthermore fix $F$ and $\mathcal{R}$ as above that satisfy (D.1.1) and define further

$$
\tau=\inf \left\{n: f_{n} \notin \mathcal{R}\right\}
$$

the first exit time of the process $f_{n}=y(n)$ defined in Section E. What is important for us is then the following

Lemma. Let $F, \mathcal{R}, \tau$ be as above. Then the process $u_{n}=F\left(f_{n \wedge \tau}\right)$ is a submartingale.

In our applications the martingale $f_{n}$ will be one of the two martingales $y(n)$ or $y\left(T_{n}\right)$ of Section $\mathrm{E}$ and then we deduce that for any starting probability of the diffusion $(x(t), t>0)$ in Section A the process $u_{n}=F\left(f_{n \wedge \tau}\right)$ is a submartingale.

The proof of this lemma is straight forward and was given in [13, II, Section 4], it will therefore be omitted.

We shall now explain a general procedure that allows us to analyze the convolution $F *(\delta-\mu)$ for any $\mu \in \mathbb{P}\left(\mathbb{R}^{n}\right)$ with $\int x d \mu(x)=0$. Towards that we shall use the Taylor development at $x \in \mathbb{R}^{n}$ and write

$$
F *(\mu-\delta)(x)=2 \sum_{i, j} \int(1-t) F_{i, j}(x+t y) y_{i} y_{j} d \mu(y) d t
$$


where $F_{i, j}=\partial^{2} F / \partial x_{i} \partial x_{j}$, we assume here that $F$ is sufficiently differentiable and that all the above integrals $\left(y \in \mathbb{R}^{n}, 0<t<1\right)$ converge absolutely.

We shall modify the above expression as follows

$2 \sum_{i, j} \int(1-t) F_{i, j}(x+t y) \frac{y_{i}}{|y|} \frac{y_{j}}{|y|} d \nu(y) d t=2 \sum_{i, j} \int F_{i, j}(x+y) \frac{y_{i}}{|y|} \frac{y_{j}}{|y|} d \lambda(y)$

where $d \nu(y)=|y|^{2} d \mu(y)$ and $d \lambda$ is the image of the measure (1 $t) d t \otimes d \nu$ by the mapping $(t, y) \rightarrow t y$. We shall assume throughout that $\int|y|^{2} d \mu(y)<+\infty$ so that $\lambda$ is a bounded measure. Quite generally for any measure $\lambda \in \mathbb{P}\left(\mathbb{R}^{n}\right)$ and any matrix $\left(h_{i j}\right)=H$ we shall introduce the notation

$$
\lambda \# H(x)=\int h_{i j}(x+y) \frac{y_{i}}{|y|} \frac{y_{j}}{|y|} d \lambda(y) .
$$

With the above notation we have therefore

$$
F *(\mu-\delta)(x)=2 \lambda \# \operatorname{Hess}(F)(x),
$$

where $\operatorname{Hess}(F)=\left(F_{i j}\right)$ denotes the Hessian matrix of $F$.

For the new measure $\lambda$ we can no longer assert that it is centered and its baricenter $\bar{\lambda}=\int x d \lambda$ may not be 0 . Indeed this is not in general true even for the measure $\nu$. Let us make the additional hypothesis that

$E(\mu)=\left(\int x_{i} x_{j} d \mu(x) ; i, j=1, \ldots, n\right) \in \mathcal{P}_{a}, \quad \int|x|^{2+\delta} d \mu(x) \leq C$,

for some $C, \delta>0, a \geq 1$. It is then easy to see that there exist $0<\varepsilon \ll 1$ and $R \gg 1$ such that

(D.1.2) $\mu\left\{x:\left|x-x_{0}\right| \leq R-\varepsilon\right\} \geq \varepsilon \quad$ for all $x_{0} \in \mathbb{R}^{n},\left|x_{0}\right|=R$.

Furthermore $R, \varepsilon$ only depend on $C, \delta, a$. This means that the measures $\mu_{x_{0}, R}=\chi_{\left\{\left|x-x_{0}\right|<R-\varepsilon\right\}} \mu \leq \mu\left(\left|x_{0}\right|=R\right)$ all satisfy $\left\|\mu_{x_{0}, R}\right\| \geq \varepsilon$. It follows also that the measures $\lambda_{x_{0}, R}$ that we can associate to $\mu_{x_{0}, R}$ by the same procedure satisfy $\left\|\lambda_{x_{0}, R}\right\| \geq \varepsilon^{3}$. In other words, the property (D.1.2) is "inherited" by $\lambda$ and can be used as a substitute of $\bar{\lambda}=0$. This point will be used at the end of Section F below.

Let us now give the proof of (D.2) and to make the argument that follows clearer let us assume first that $\mu \in \mathbb{P}\left(\mathbb{R}^{n}\right)$ as above is 
compactly supported and that its moment matrix satisfies $E(\mu)=$ $\left(\int x_{i} x_{j} d \mu(x) ; i, j=1, \ldots, n\right) \in \mathcal{P}_{a}$ for some $a>1$. The condition that we have imposed on the moment matrix is then equivalent to the fact that there exists $\varepsilon>0$ such that for any $(n-1)$-dimensional subspace $H \subset \mathbb{R}^{n}$ we have

$$
\mu\{x: \operatorname{dist}(x, H) \geq \varepsilon\} \geq \varepsilon .
$$

Since $\operatorname{supp} \mu$ is compact it is easy to see that the measure $\lambda$ that corresponds to the above $\mu$ has the same property (D.1.3) and therefore it follows that the corresponding moment matrix

$$
E(\lambda)=\left(\int x_{i} x_{j} d \lambda(x) ; i, j=1, \ldots, n\right) \in \mathcal{P}_{b}
$$

for some $b>1$.

We shall apply the above considerations to the function $F(x)=$ $F_{\nu, k}(x)$ of Section C. By the proposition of Section F and (D.1.4) we deduce that for any $\mu$ as above and $|x|$ appropiately large we have

$$
F *(\delta-\mu)(x) \leq 0,
$$

and our lemma applies. An easy adaptation of the argument (A.2.4), (A.2.5), (A.2.6) completes then the proof of the assertion (D.2) for the case $p=+\infty$ in (D.0.2).

There are several ways of getting rid of the compactness of the support in (D.1.4) since we shall not need optimal results, let us proceed as follows: Suppose as above that $E(\mu) \in \mathcal{P}_{a}$ and that supp $\mu \subset\{|x| \leq$ $R\}, R \gg 1$. It is easy to see (e.g. by scaling) that $E(\lambda) \in \mathcal{P}_{b}$ where $b \sim$ $\mathbb{R}^{2}$ (if $a$ is fixed). Let then $\mu$ be an arbitrary measure that is assumed to admit a high enough moment $E_{N}=\int|x|^{N} d \mu<+\infty(N \gg 1)$ and let us denote by $\mu_{R}=\chi_{\{|x|>R\}} \mu$, the part of $\mu$ at $\infty$, and by $\lambda_{R}$ the measure that corresponds to $\mu_{R}$ we have then $E\left(\lambda_{R}\right)=O\left(R^{-\alpha}\right)$ for an arbitrary large $\alpha$ (provided that $N$ is high enough). We can therefore correct the contribution of $\lambda$ coming from $\mu^{R}=\chi_{\{|x|<R\}} \mu$ by $O\left(R^{-\alpha}\right)$ and obtain that $E(\lambda) \in \mathcal{P}_{b}$ with $b^{-1} \sim R^{-2}+O\left(R^{-\alpha}\right)$. For $R$ large enough we obtain thus again (D.1.4) for some $b$ that only depends on $a, N$ and $E_{N}$. Working out the exact value of $N$ is not so hard and that exact value of $N$ is not so large either.

The proof of (D.2) for general values of $p<+\infty$ in (D.0.2) can then be completed as before except that we now have to use the second 
half of Section F. For our applications however the case $p<+\infty$ is not essential. Indeed if we use the martingale $y\left(T_{n}\right)$ constructed in Section E.2 then the supports of all the measures involved are (uniformly) compact and $p=+\infty$.

\section{D.2. The radial function and the proof of (D.1) (The case $p=+\infty$ in (D.0.2)).}

Let $0 \leq \varphi \in C^{N}\left(\mathbb{R}^{n}\right)$ be radial decreasing (i.e. $\varphi(x)=\varphi(r)$, $r=|x|$ and $N$ is sufficiently large) and such that $\varphi \equiv 1$ if $|x|<1 / 2$ and $\varphi \equiv 0$ if $|x| \geq 1, \varphi>0$ if $|x|<1$. Let us further assume that $\varphi(x)=(1-r)^{\nu}$ for $3 / 4 \leq r \leq 1$ and $\nu=4,6, \ldots$ some appropiately large even integer. The above function is clearly not convex (if $n \geq 2$ it is not even convex in some Nhd of the unit sphere $r=1$ ). Let $\operatorname{Hess}(\varphi)=\left(\partial^{2} \varphi / \partial x_{i} \partial x_{j}\right)$ be the corresponding Hessian matrix. This matrix can easily be diagonalized and an easy calculation shows that for $|x| \sim 1$ we have

$$
\operatorname{Hess} \varphi=\nu(\nu-1)(1-r)^{\nu-2} \rho \otimes \rho-c \nu(1-r)^{\nu-1} \theta_{j} \otimes \theta_{j},
$$

where $\rho(x)$ is the unit vector along the radius $\overline{O x}$ and $\theta_{j}(x)(j=$ $1, \ldots, n-1)$ are an orthonormal complement of $\rho(x)$ (tangent to the sphere $\{y:|y|=|x|\}$.

The crucial fact in the structure of the above Hessian is that for each $r_{0}<1$ if we add some appropiately large multiple of $\varphi$ we obtain a positive matrix

$$
\operatorname{Hess} \varphi+C \varphi I \gg 0, \quad|x|<r_{0}
$$

By scaling therefore $\varphi_{M}(\cdot)=\varphi(\cdot / M)(M>0)$ we obtain

$$
\operatorname{Hess} \varphi_{M}+C M^{-2} \varphi_{M} I \gg 0, \quad|x|<M r_{0} \text {. }
$$

If we use the second order Taylor development of $\varphi_{M}$ we obtain therefore that

$$
\varphi_{M} *(\mu-\delta)(x) \geq-C M^{-2} \int \varphi_{M}(x+y) d \lambda(y), \quad|x|<M r_{0},
$$

where $\mu$ and $\lambda$ are as in Section (D.1) and are compactly supported since $p=+\infty$, and $\mu$ satisfies [(D.1.3) of Section D.1]. Then $\nu$ and $\lambda$ 
also satisfy (D.1.3) and since $\varphi_{M}(r)$ is decreasing it follows that there exist $M_{0}, c_{0}>0$ such that

$$
\varphi_{M} *(\mu-\delta)(x) \geq-c_{0} M^{-2} \varphi_{M}(x), \quad M \geq M_{0},|x|<M r_{0}
$$

The next observation is that for all $a>1$ there exists $\varepsilon$ such that if $1-r_{1}<\varepsilon$ then Hess $\varphi \in \mathcal{S}_{a},|x|>r_{1}$ therefore also Hess $\varphi_{M} \in \mathcal{S}_{a}(M>$ $\left.0,|x|>r_{1} M\right)$. In informal terms this says that, near the boundary, $\varphi$ "looks more and more like a convex function". In fact by an elementary calculation, that is best carried out by drawing a few pictures, we see that (D.2.1) holds (with $c_{0}=0$ ) for $|x| \geq r_{1} M$ provided that $1-r_{1}$ is small enough and $M$ large enough. The final conclusion is therefore that (D.2.1) holds for all $x \in \mathbb{R}^{n}$.

From the estimate (D.2.1) we deduce that there exist $M_{1}, c>0$ such that

$$
\varphi_{M} * \mu(x) \geq e^{-c M^{-2}} \varphi_{M}(x), \quad x \in \mathbb{R}^{n}, M \geq M_{1}
$$

To finish the proof of (D.1) let us set $T=\inf \left\{j: f_{j} \geq M\right\}, f_{j}^{*}=f_{j \wedge T}$ and let us apply (D.2.2) with $\mu=\mu_{j, x}$ as in (D.0.3). We obtain

$$
\mathbb{E}\left[\varphi_{M}\left(f_{j}^{*}\right) / / \mathcal{F}_{j-1}\right] \geq e^{-c M^{-2}} \varphi_{M}\left(f_{j-1}^{*}\right)
$$

which by iteration gives

$$
\mathbb{E}\left[\varphi_{M}\left(f_{n}^{*}\right)\right] \geq e^{-c n / M^{2}}, \quad n \geq 1, M \geq M_{1} .
$$

From (D.2.3) (D.1) follows at once. It has thus been shown that (D.1) holds if $p=+\infty$ in (D.0.2). The above argument can be adapted to deal with $p<+\infty$, the proof will be omitted since this is not essential for us.

\section{E. Discretising the continuous time martingale.}

\section{E.1. The deterministic discretisation $t=1,2, \ldots$.}

We shall preserve all the notations of Section A and recall (Section A.1) that $y(t)=z(t)+\psi(k(t))+C \in \mathbb{R}^{n}$ is a continuous time martingale. It follows in particular that $f_{j}=y(j)(j \geq 0)$ is a discrete time 
martingale (unlike Section D.0 $f_{0}=C$ which is not necessarily 0 ) our purpose is to examine

$$
\mathbb{P}\left[f_{j}-f_{j-1} \in d y / / f_{1}=x_{1}, \ldots, f_{j-1}=x_{j-1}\right]=p_{j}(y ; x) d y,
$$

for $x=\left(x_{1}, \ldots, x_{j-1}\right), y \in \mathbb{R}^{n}$. To be formally correct the above probability density is only defined $x$ almost surely and is a measure $d \mu_{x}(y)$. The above abusive notation will be justified by what follows. What is clear by the martingale property is that

$$
\int y d \mu_{x}(y)=0, \quad j=0,1, \ldots, x \in \mathbb{R}^{n} .
$$

We shall show then that there exist $C, C_{1}>0$ such that we have (uniformly in $x$ )

$$
C_{1}^{-1} \exp \left(-C|y|^{2}\right) \leq p_{j}(y, x) \leq C_{1} \exp \left(-\frac{|y|^{2}}{C}\right), \quad y \in \mathbb{R}^{n}
$$

It is essentially this estimate that justifies our previous abusive notation. It is clear that it suffices to prove the same Gaussian estimates for the "finer" conditional probabilities with respect to the fields $\mathcal{T}_{j}=$ $\mathcal{T}\{z(t) ; t \leq j-1\}$. By the Markov property we must therefore consider the conditional properties

$$
\mathbb{P}\left[f_{j}-f_{j-1} \in d y / /(z(j-1), k(j-1))=x\right]=\tilde{p}_{j}(y ; x) d y,
$$

for $y \in \mathbb{R}^{n}, x=(z, k) \in X=\mathbb{R}^{n} \times K$. These new Gaussian estimates can be deduced from the Gaussian estimates for the diffusion kernel $q_{t}\left(x_{1}, x_{2}\right),\left(t>0, x_{i}=\left(z_{i}, k_{i}\right) \in X, i=1,2\right)$ of the diffusion $\Omega$ ( $c f$. Section A.0)

$$
C_{1}^{-1} \exp \left(-C\left|z_{1}-z_{2}\right|^{2}\right) \leq q_{1}\left(x_{1}, x_{2}\right) \leq C_{1} \exp \left(-\frac{\left|z_{1}-z_{2}\right|^{2}}{C}\right)
$$

To deduce (E.1.1) from (E.1.2) one simply "integrates" along the fibers

$$
F_{y}=\{(z, k) \in X: z+\psi(k)=y\} .
$$

The upper Gaussian estimate (E.1.2) is perfectly standard and follows from the more general $\left(C^{\infty}\right.$-manifold) upper Gaussian estimates for subelliptic operators and the intrinsic distance that they induce ( $c f$. 
[1]). The lower estimates makes essential use of the left invariance of $D$ and the corresponding scaled (for "small balls" but spacially uniform) Harnack estimate. The argument is an easy adaptation of [19]. Alternatively, if the reader is not prepared to either believe or verify for himself the above argument, he could refer to [1] where the above lower estimate is explicitely proved for Lie groups $G$ and left invariant operators. The diffusion $\Omega$ that we will be considering is non other than the diffusion that in Section 2 is induced on $R \times K$ by the corresponding diffusion in our original group $G$. The lower Gaussian estimate (E.1.2) can then easily be picked up by the corresponding estimate in that group. The verification will be left to the reader.

The reader should also observe that the above lower Gaussian estimate is not essential for us here. Indeed the reason that we need these estimates is that we have to show that the above martingale $f_{j}=y(j)$ satisfies the conditions of Section D.0. For this it suffices to have the upper Gaussian estimate (E.1.1) which guarantees the moment condition (D.0.2) and a much weaker lower estimate of the form

$$
\tilde{p}_{j}(y, x) \geq \varepsilon, \quad|y|<\varepsilon,
$$

for some $\varepsilon>0$. This is guaranteed by the uniform Harnack estimate on $X$ for the operator $D$.

\section{E.2. The optional time discretisation.}

There is an alternative way to discretise the time parameter of the martingale $y(t),(t>0)$. Let $T_{0}=0$ and

$$
T_{1}=\inf \{t /|z(t)-z(0)| \geq C\}, \quad T_{j}=\inf \left\{t /\left|z(t)-z\left(T_{j-1}\right)\right| \geq C\right\},
$$

for $j=2,3, \ldots$, and some large $C>0$. We can set then $f_{j}=y\left(T_{j}\right)$, $(j=1,2, \ldots)$ which is now a martingale as in Section D.0 with the additional property that the martingale differences $d_{j}=f_{j}-f_{j-1} \in L^{\infty}$ are uniformly bounded. For this new martingale we shall define again

$$
\mathbb{P}\left[f_{j}-f_{j-1} \in d y / / x\left(T_{j-1}\right)=\left(z\left(T_{j-1}\right), k\left(T_{j-1}\right)\right)=x\right]=d \tilde{\mu}_{x}(y),
$$

for $j=1,2, \ldots, x \in X=\mathbb{R}^{n} \times K$, and

$$
\mathbb{P}\left[f_{j}-f_{j-1} \in d y / / f_{1}=x_{1}, \ldots, f_{j-1}=x_{j-1}\right]=d \mu_{x}(y),
$$


for $j=1,2, \ldots, x_{1}, \cdots \in \mathbb{R}^{n}$, and we shall show that the measures $\mu_{x}$ satisfy the covariance condition of Section D.0 uniformly in $j \geq 1$ and $x \in \mathbb{R}^{n}$. For this it clearly suffices to prove the corresponding covariance condition (D.0.4) for the measures $\tilde{\mu}_{x}$. To see this fact one simply has to understand what a subelliptic diffusion means. The best way to analyze this situation is to work with the "trajectories" of the diffusion $\Omega$ ( $c f$. Section A.0).

Indeed if $x, y \in \Theta \subset X$ where $\Theta$ is some open set of $X$ then

$$
\begin{gathered}
\mathbb{P}_{0}\left[x\left(T_{j-1}+t\right) \in \Theta, 0<t<t_{0} ; \operatorname{dist}\left(x\left(T_{j-1}+t_{0}\right), y\right)<\varepsilon / / x\left(T_{j-1}\right)=x\right] \\
=\mathbb{P}_{x}\left[x(t) \in \Theta, 0<t<t_{0} ; \operatorname{dist}\left(x\left(t_{0}\right), y\right)<\varepsilon\right]>0
\end{gathered}
$$

for any $t_{0}$ and $\varepsilon>0$. This is a basic consequence of the subellipticity of the operator $D$ and follows from the smoothness of the heat diffusion kernel and elementary (if lenghty and tedious) considerations that will be left for reader.

For fixed $x$ and $j$ therefore, by appropiately chosing $t_{0}$ and $y$ we see that measure $\mu_{x}(d y)$ charges positively (and in and uniform fashion with respect to $x$ and $j$ ) a whole family of small discs around $x$. Furthermore there are enough of these discs on every direction as we go away from $x$ to guarantee the covariance condition (D.0.4) for $\tilde{\mu}_{x}$. The details will be left to the reader.

\section{F. The geometry of the Hessian.}

\section{F.1. Dimension $=n=2$.}

To see clearly what is involved we shall first consider the case of $\mathbb{R}^{2}$ (i.e. $n=$ dimension $=2$ ). We shall preserve all the notations of the previous sections and translate the $\theta$-variable (now of course the polar coordinates are $\left.(x, y)=(r, \theta) \in \mathbb{R}^{2}\right)$ by $\theta_{0}=\alpha$ so that the $x$-axis becomes one of the two edges of the wedge $C_{\alpha}$, and $\{x \geq 0, y=0\}=$ $\{r \geq 0, \theta=0\}$.

For these coordinates we have

$$
\frac{\partial}{\partial x}=\cos \theta \frac{\partial}{\partial r}-\sin \theta \frac{1}{r} \frac{\partial}{\partial \theta}, \frac{\partial}{\partial y}=\sin \theta \frac{\partial}{\partial r}+\cos \theta \frac{1}{r} \frac{\partial}{\partial \theta} .
$$

For $\theta \sim 0(\theta>0)$ and $F=F_{\nu, k}$ as in Section $C$ we obtain by a straight 
forward calculation that

$$
\text { Hess } F=r^{\nu-2} \theta^{k-2}\left(\begin{array}{cc}
\nu^{2} \theta^{2} & \nu k \theta+\nu^{2} \theta^{3} \\
\nu k \theta+\nu^{2} \theta^{3} & k^{2}+(2 \nu k+\nu-k) \theta^{2}+\nu^{2} \theta^{4}
\end{array}\right)
$$

$$
=r^{\nu-2} \theta^{k-2} \mathcal{H}
$$

where each coefficient of the matrix $\mathcal{H}$ has to be multiplied in addition by a factor $=1+O\left(\theta^{2}\right)+O(1 / k)+O(k / \nu)$, and in the considerations where $\mathcal{H}$ is used we shall assume throughout that $0<\theta<\varepsilon_{0} \ll 1$, $1 \ll k, 1 \ll \nu / k$.

For a $\theta$ that it is not close to 0 and $F=r^{\nu} u(\theta)$ we also have the following expression of the Hessian

$$
\text { Hess } \begin{aligned}
F & =\nu(\nu-1) r^{\nu-2} u(\theta)\left(\begin{array}{cc}
\cos ^{2} \theta & \cos \theta \sin \theta \\
\cos \theta \sin \theta & \sin ^{2} \theta
\end{array}\right) \\
& =\nu(\nu-1) r^{\nu-2} u(\theta) \tilde{\mathcal{H}},
\end{aligned}
$$

where every coefficient of the matrix $\tilde{\mathcal{H}}$ has to be multiplied by a factor of the form $(1+O(1 / \nu))$ with a $O(\cdot)$ that depends of course on the particular chice of $u$.

Let now quite generally $K=\left(k_{i, j}(x)\right) \in \mathcal{S}, x \in \mathbb{R}^{n}$, denote an arbitrary matrix and let $\mu \geq 0$ denote some non negative measure on $\mathbb{R}^{n}$. We shall use then the same notation as in Section D.1

$$
(\mu \# K)(x)=\sum_{i, j} \int k_{i j}(x+z) \frac{z_{i}}{|z|} \frac{z_{j}}{|z|} d \mu(z),
$$

where we shall assume that all the above integrals converge absolutely. I shall denote

$$
E_{i j}=\int x_{i} x_{j} d \mu(x)
$$

We have then

Proposition. Let $\mu$ be as above and let us assume that $E=\left(E_{i j}\right) \in \mathcal{P}_{b}$ for some $b>1$ and that $\mu$ is a probability measure supported in the unit ball: $\operatorname{supp} \mu \subset\left\{x \in \mathbb{R}^{n}:|x| \leq 1\right\}$. Then there exists a choice of $\nu, k$ and some $r_{0}>0$ such that

$$
\left(\mu \# \operatorname{Hess} F_{\nu, k}\right)(x) \geq 0, \quad x \in \mathbb{R}^{n},|x| \geq r_{0} \text {. }
$$


The proof is elementary but lenghty. Before we give the proof we shall explicitely state the three basic properties of Hess $F$ that make the things work. The proof of this properties will be left to the reader.

a) Using the notation of Section C.1 we shall diagonalize the matrix $\mathcal{H}$ at every $z \in \mathbb{R}^{2}$

$$
\mathcal{H}=\lambda_{1} e_{1} \otimes e_{1}+\lambda_{2} e_{2} \otimes e_{2},
$$

where $\left(e_{1}, e_{2}\right)$ is an orthonormal basis of $\mathbb{R}^{2}$ and where $\lambda_{1} \geq 1 / 2$ provided that $0<\theta<\varepsilon_{0}$ for $\varepsilon_{0}$ small enough and $k, \nu / k$ are large enough. Furthermore by direct computations or by the considerations of Section $\mathrm{C}$ we see that for all $a>1$ there exists $t_{0}, k_{0}$ such that $\lambda_{1} \geq a\left|\lambda_{2}\right|$ for $\nu / k \geq t_{0}, k \geq k_{0}$.

b) Let us assume that $\nu$ and $k$ are fixed, then $\lambda_{i}(z)$ and $e_{i}(z)$ are continuous functions of $\theta$. By the uniform continuity and the fact that $\theta \lesssim y /|z|$ we see therefore that for all $\delta>0$ there exists $r_{0}=r_{0}(\delta ; \nu, k)$ such that

$\left|\lambda_{i}\left(z_{1}\right)-\lambda_{i}\left(z_{2}\right)\right| \leq \delta, \quad\left|e_{i}\left(z_{1}\right)-e_{i}\left(z_{2}\right)\right| \leq \delta, \quad\left|z_{1}-z_{2}\right| \leq 10,\left|z_{i}\right| \geq r_{0}$.

c) Let us again fix $\nu$ and $k$. Near $\theta=0$ we have

$$
\mu \# \text { Hess } F_{\nu, k}=\left.\mu \# r^{\nu-2} \theta^{k-2} \mathcal{H}\right|_{\theta=0}+\text { Error . }
$$

Let $A>0$ be fixed for $z_{1}=\left(x_{1}, y_{1}\right)$ with $\left|y_{1}\right| \leq A$ and $x_{1}$ large enough. We have

$$
\begin{aligned}
\left(\left.\mu \# r^{\nu-2} \theta^{k-2} \mathcal{H}\right|_{\theta=0}\right)\left(z_{1}\right) & =k^{2} \int r^{\nu-2} \theta^{k-2} \frac{y^{2}}{|z|^{2}} d \mu(z) \\
& =x_{1}^{\nu-2-k}\left(c_{0}+O\left(1 / x_{1}\right)\right),
\end{aligned}
$$

where $c_{0}>0$. To prove that $c_{0} \neq 0$ we use already the hypothesis $E \in \mathcal{P}_{b}$. By an easy calculation on the other hand one sees that the "error" in (F.1.4) is $O\left(x_{1}^{\nu-3-k}\right)$ because all but the $k^{2}$ terms of $\mathcal{H}$ involve higher powers of $\theta$.

The conclusion is that for fixed $\nu, k$ and $A$ we can find $B>0$ such that our estimate (F.1.3) holds in the region

$$
R_{A, B}=\{z=(x, y): x \geq B, y \leq A\} .
$$


What is furthermore important about the region $R_{A, B}$ is that (again with $\nu, k$ fixed) for any $\delta>0$ we can find $A$ and $r_{0}$ large enough so that the co-factor of $\mathcal{H}$ in (F.1.1) $\varphi=\varphi(z)=r^{\nu-2} \theta^{k-2}$ satisfies

$$
1-\delta \leq \frac{\varphi\left(z_{1}\right)}{\varphi\left(z_{2}\right)} \leq 1+\delta, \quad\left|z_{1}-z_{2}\right| \leq 1, \quad\left|z_{1}\right| \geq r_{0}, z_{1} \notin R_{A, r_{0}} .
$$

In other words that cofactor varies as slowly as we like outside the regions $R_{A, B}$ for large $|z|$.

We shall now procede with the proof of the proposition. Because of c) above, it remains to prove (F.1.3) in the region outside $R_{A}, r_{0}$. With the notations already introduced we have then

$$
\mu \# \operatorname{Hess} F_{\nu, k}\left(z_{0}\right)=\int \psi\left(z_{0} ; z\right) d \mu\left(z-z_{0}\right),
$$

where

$$
\psi\left(z_{0}, z\right)=\varphi(z)\left(\lambda_{1}(z)\left\langle e_{1}(z), \frac{z-z_{0}}{\left|z-z_{0}\right|}\right\rangle^{2}+\lambda_{2}(z)\left\langle e_{2}(z), \frac{z-z_{0}}{\left|z-z_{0}\right|}\right\rangle^{2}\right)
$$

and where $z_{0}$ lies in the region $0<\theta<\varepsilon_{0}$.

For any $\varepsilon \geq 0$ let us denote

$$
B_{\varepsilon}\left(z_{0}\right)=\left\{z:\left|z-z_{0}\right| \leq 1,\left|\left\langle e_{1}\left(z_{0}\right), z-z_{0}\right\rangle\right| \geq \varepsilon\left|z-z_{0}\right|\right\}
$$

so that $B_{0}$ is the unit ball centered at $z_{0}$. Let us now fix $\varepsilon>0$, we can then find $t_{0}, k_{0}$ so that

$$
\lambda_{1}(z)\left\langle e_{1}\left(z_{0}\right), \frac{z-z_{0}}{\left|z-z_{0}\right|}\right\rangle^{2} \geq 10^{100}\left|\lambda_{2}(z)\right|\left\langle e_{2}\left(z_{0}\right), \frac{z-z_{0}}{\left|z-z_{0}\right|}\right\rangle^{2},
$$

for $\nu / k \geq t_{0}, k \geq k_{0}, z \in B_{\varepsilon}$. Observe that here the argument $z$ has been frozen to $z=z_{0}$ in $e_{i}(\cdot)$. We shall fix $k=k_{0}$ and will not change it anymore. Using b) for every $\nu \geq \nu_{0}\left(k_{0}\right)$ we can find $r_{0}=r_{0}(\nu)$ such that

$$
\lambda_{1}(z)\left\langle e_{1}(z), \frac{z-z_{0}}{\left|z-z_{0}\right|}\right\rangle^{2} \geq 10^{10}\left|\lambda_{2}\left(z^{\prime}\right)\right|\left\langle e_{2}\left(z^{\prime}\right), \frac{z^{\prime}-z_{0}}{\left|z^{\prime}-z_{0}\right|}\right\rangle^{2},
$$

for $\nu \geq \nu_{0},\left|z-z^{\prime}\right| \leq 10,|z| \geq r_{0}(\nu), z \in B_{\varepsilon}$. Using c) and the slow variation of $\varphi$ outside $R_{A}, r_{0}$ we see that there exists $A>0$ such that for all $\nu \geq \nu_{0}$ large enough there exists $r_{1}(\nu)>0$ such that

$$
\begin{aligned}
\varphi(z) \lambda_{1}(z) & \left\langle e_{1}(z), \frac{z-z_{0}}{\left|z-z_{0}\right|}\right\rangle^{2} \\
& \geq 10^{5} \varphi\left(z^{\prime}\right)\left|\lambda_{2}\left(z^{\prime}\right)\right|\left\langle e_{2}\left(z^{\prime}\right), \frac{z^{\prime}-z_{0}}{\left|z^{\prime}-z_{0}\right|}\right\rangle^{2},
\end{aligned}
$$


for $\left|z-z^{\prime}\right| \leq 10, z=(x, y) \in \mathbb{R}^{2},|z| \geq r_{1}(\nu),|y|>A, z \in B_{\varepsilon}$. At this point one should remember that we are working close to the edge of $C_{\alpha}$ i.e. in a range $0<\theta<\varepsilon_{0}$ for some small $\varepsilon_{0}$. The estimate (F.1.5) will now be used in conjunction with the fact that because of our hypothesis $E \in \mathcal{P}_{b}$, for $\varepsilon>0$ small enough, we have $\mu\left(B_{0} \backslash B_{\varepsilon}\right) \leq 10^{-10}$ (cf. Section D.1). If we integrate (F.1.5) against $d \mu\left(z-z_{0}\right) d \mu\left(z^{\prime}-z_{0}\right)$ we obtain that for all $\nu \geq \nu_{0}$ there exists $r_{0}=r_{0}(\nu)$ such that

$$
\mu \# \text { Hess } F_{\nu, k} \geq 10^{-2} \varphi\left(z_{1}\right) \lambda_{1}\left(z_{1}\right)\left\langle e_{1}\left(z_{1}\right), \frac{z_{1}-z_{0}}{\left|z_{1}-z_{0}\right|}\right\rangle^{2},
$$

for $\nu \geq \nu_{0}, z_{i}=\left(x_{i}, y_{i}\right) \in \mathbb{R}^{2}, i=0,1,\left|z_{0}-z_{1}\right| \leq 1, z_{1} \in B_{0}$, $\left|z_{i}\right| \geq r_{0}(\nu), y_{i} \geq 2 A+10$. In particular (F.1.6) holds for $z_{1}=z_{0}$ which together with c) shows that for $k=k_{0}$ and every $\nu \geq \nu_{0}$ there exists $r_{0}=r_{0}(\nu)$ such that

$$
\mu \# \operatorname{Hess} F_{\nu, k_{0}}(z) \geq 0, \quad \nu \geq \nu_{0}, 0<\theta<\varepsilon_{0},|z| \geq r_{0}(\nu) .
$$

To finish the proof of the proposition, since $k=k_{0}$ has been fixed, we shall complete the definition of $u(\theta)$ in $F_{\nu, k}=r^{\nu} u(\theta)$ and use the formula (F.1.2) for the Hessian. Using that formula and the same method (this method now applies much easier. Indeed we do not have the edge, where the co-factor $\theta^{k-2}$ vanishes, to worry about!) we finally see again that there exists $\nu_{1} \geq 0$ such that for all $\nu \geq \nu_{1}$ there exists $r_{0}=r_{0}(\nu)$ such that

$$
\mu \# \operatorname{Hess} F_{\nu, k_{0}}(z) \geq 0, \quad \nu \geq \nu_{1}, \quad \varepsilon_{0} \leq|\theta| \leq 2 \theta_{0}-\varepsilon_{0},|z| \geq r_{1}(\nu) .
$$

If we combine this (F.1.7) we see that we have a proof of the proposition.

The rest of this section will be devoted to the proof of the proposition when the support of $\mu$ is not compact under some additional conditions. This is interesting on its own right but is not essential for the rest of the paper. We start by extracting as much as possible from our previous argument.

Let $\xi_{i}(z), i=1,2$ be the two eigenvalues of Hess $F_{\nu, k}$ with $\left|\xi_{1}\right| \geq\left|\xi_{2}\right|$ (when $0<\theta<\varepsilon_{0}$ with our previous notations we have of course $\xi_{i}=$ $\left.r^{\nu-2} \theta^{k-2} \lambda_{i}, i=1,2\right)$. By analizing our previous argument we see that if $\operatorname{supp} \mu \subset[|x| \leq \beta]$ for some fixed $\beta$, then we can find $k_{0}, \nu_{0}, c_{0}>0$ such that for all $\nu \geq \nu_{0}$ there exists $r_{0}=r_{0}(\nu)$ such that

$$
\begin{aligned}
\mu \# F_{\nu, k_{0}}(z) & \geq c_{0} \int \xi_{1}(z+x) d \mu(x) \\
& =c_{0}\left\|\operatorname{Hess} F_{\nu, k_{0}}\right\| * \mu(z), \quad|z| \geq r_{0}(\nu) .
\end{aligned}
$$


What is important is to analyze the dependence of $c_{0}$ on the parameters of the construction. Following the construction through and preserving the same notations we see that if $\beta$ and $b$ are kept fixed then we can set $c_{0} \geq C_{1}(b, \beta) \varepsilon^{2}$. This $\varepsilon>0$ (which is assumed small) is the $\varepsilon$ that was used in the definition of $B_{\varepsilon}\left(z_{0}\right)$.

The important aspect of the estimate (F.1.8) is that it is "scale invariant". First of all it is clear that nothing changes if we replace $F_{\nu, k}$ by $C F_{\nu, k}$ some constant multiple of $F_{\nu, k}$. Because of the homogeneity of $F$ it follows that we can replace $\mu$ by any $\mu_{\rho}$ where $\mu_{\rho}$ is the image of the measure $\mu$ by the dilatation $\rho: x \mapsto \rho x$ in $\mathbb{R}^{2}$. Clearly the dilatation $\rho$ replaces $\beta$ by $\rho \beta$ and $b$ by $\max \left\{\rho^{2}, \rho^{-2}\right\} b$. It follows in particular that we cannot shrink a large $\beta$ to 1 without at the same time having $b$ go to $\infty$.

The dependence of $c_{0}$ on $b$, for fixed say $\beta=1$, must therefore be examined. That dependence is of course picked up by the condition $\mu\left(B_{0} \backslash B_{\varepsilon}\right) \leq 10^{-10}$. This gives $\varepsilon \sim b^{-1 / 2}$ and by the above dilatation argument we conclude that for fixed $b>1, c_{0} \sim 1 / \beta$. More explicitely if $b$ is fixed, we can choose $k_{0}, \nu_{0}, c$ such that for all $\nu \geq \nu_{0}$ and all $\beta>1$ there exists $r_{0}=r_{0}(\nu, \beta)$ such that

$$
\begin{aligned}
\mu \# \operatorname{Hess} F_{\nu, k_{0}}(z) & \geq \frac{c}{\beta} \int \xi_{1}(z+x) d \mu(x) \\
& =\frac{c}{\beta} \int\left\|\operatorname{Hess} F_{\nu, k_{0}}(z+x)\right\| d \mu(x),
\end{aligned}
$$

for $\mu \in \mathbb{P}[|x| \leq \beta], E(\mu) \in \mathcal{P}_{b},|z| \geq r_{0}(\nu, \beta)$. The only thing that really counts in (F.1.9) is that the dependence of the co-factor of the integrals is polynomial in $\beta$. A co-factor of the form $c / \beta^{10}$ would have been just as good for our purposes.

With the help of (F.1.9) we shall generalise our proposition to measure that are not compactly supported. To do this we have to go back to Section D.1 and to start from some $\mu \in \mathbb{P}\left(\mathbb{R}^{n}\right)$ such that

$$
\int|x|^{N} d \mu(x)<+\infty
$$

and such that

$$
\bar{\mu}=\int x d \mu(x)=0, \quad\left(\int x_{i} x_{j} d \mu(x) ; i, j=1, \ldots, n\right) \in \mathcal{P}_{c},
$$

for some large $N$ large enough and some $c>1$. We shall next consider the measure $\lambda$ that corresponds to $\mu$ as in Section D.1. It is for that 
measure $\lambda$ that we shall need to generalize our proposition and prove that

$$
\left(\lambda \# \operatorname{Hess} F_{\nu, k_{0}}\right)(x) \geq 0, \quad x \in \mathbb{R}^{n},|x| \geq r_{0} .
$$

This new measure $\lambda$ also satisfies

$$
\begin{gathered}
\int|x|^{N} d \lambda(x)<+\infty, \\
E=\left(\int x_{i} x_{j} d \lambda(x) ; i, j=1, \ldots, n\right) \in \mathcal{P}_{b},
\end{gathered}
$$

for some $N$ as large as we like and some $b>1$ (but does not necessarily satisfy $\left.\bar{\lambda}=\int x d \lambda(x)=0\right)$.

The next step is to examine $\|$ Hess $F_{\nu, k} \|$ as obtained from the two formulas (F.1.1), (F.1.2). An easy calculation gives

$C_{2}\left(k^{2} r^{\nu-2} \theta^{k-2}+\nu^{2} r^{\nu-2} \theta^{k}\right) \leq\left\|\operatorname{Hess} F_{\nu, k}\right\| \leq C_{1}\left(k^{2} r^{\nu-2} \theta^{k-2}+\nu^{2} r^{\nu-2} \theta^{k}\right)$

valid in the $1 / 2-C_{\alpha}$ cone $\left(0<\theta<\theta_{0}\right)$ that is closest to the edge $\theta=0$. It follows that in that region if we use cartesian coordinates we have

$$
\begin{aligned}
\left\|\operatorname{Hess} F_{\nu, k}\right\| \sim & k^{2}\left(x^{\nu-k} y^{k-2}+x^{-k+2} y^{\nu+k-4}\right) \\
& +\nu^{2}\left(x^{\nu-k-2} y^{k}+x^{-k} y^{\nu+k-2}\right) .
\end{aligned}
$$

If we combine the two $1 / 2$ subregions of $C_{\alpha}$ we see that if we denote by $\xi=\xi(z)=\operatorname{dist}\left(z, \partial C_{\alpha}\right)$ we obtain the estimate

$$
\begin{aligned}
\left\|\operatorname{Hess} F_{\nu, k}\right\| \sim & k^{2}\left(x^{\nu-k} \xi^{k-2}+x^{-k+2} \xi^{\nu+k-4}\right) \\
& +\nu^{2}\left(x^{\nu-k-2} \xi^{k}+x^{-k} \xi^{\nu+k-2}\right),
\end{aligned}
$$

valid in the whole $C_{\alpha}$. Let us consider the functions $\psi_{A, B}(z)=x^{A} \xi^{B}$ $\left(z \in C_{\alpha}\right)$ and $\psi(z) \equiv 0\left(z \notin C_{\alpha}\right)$ where $(A, B)$ takes the four possible values that appear in the right hand side of (F.1.12). To prove (F.1.10) it will suffice to show that any of the above four functions $\psi_{A, B}=\psi_{\nu, k}$ has the following property: There exist $C_{1}, C_{2}>0$ that do not depend on $\nu$ (but may depend on $k$ ) such that for all $\nu \geq 1$ there exists $u_{0}(\nu)>0$ such that

$$
\int_{|z|>\beta} \psi_{\nu, k}(u+z) d \lambda(z) \leq\left(\frac{C_{2}}{\beta}\right)^{\nu / 2} \int_{|z| \leq C_{1}} \psi_{\nu, k}(u+z) d \lambda(z)
$$


for $\nu \geq 1,|u| \geq u_{0}(\nu), \beta \geq 1, u \in C_{\alpha}$. Indeed once we have (F.1.13) we shall truncate $\lambda$ at $[|z| \leq \beta]$. If we use (F.1.9) and the same correcting argument as at the end of Section D.1 we see that (F.1.10) follows.

Let us fix $R, \varepsilon>0$, let us assume that $\lambda$ satisfies (D.1.2) and let us denote

$$
m_{\nu, k}(u)=\sup _{x ;|x-u|=R}\left\{\inf _{z}\left[\psi_{\nu, k}(z):|x-z| \leq R-\varepsilon\right]\right\} .
$$

It is clear from (D.1.2) that for an appropiate $C_{1}>0$ we have then

$$
m_{\nu, k}(u) \leq C_{1} \int_{|z| \leq C_{1}} \psi_{\nu, k}(u+z) d \lambda(z) .
$$

(F.1.13) will therefore follow as soon as we can show that

$$
\int_{|z|>\beta} \psi_{\nu, k}(u+z) d \lambda(z) \leq\left(\frac{C_{2}}{\beta}\right)^{\nu / 2} m_{\nu, k}(u)
$$

with $\nu, u$ and $\beta$ as in (F.1.13). The only thing, of course, that really has to be verified in the above estimate is that the constant $C_{2}$ is uniform in $\nu$. By the structure of the above functions $\psi$ it is clear also that we can fix $A \gg R+\varepsilon$ and distinguish the following two cases.

Case 1. distance $\left(u, \partial C_{\alpha}\right)>A$. One then simply has to verify that

$$
\int_{|z|>\beta} \psi_{\nu, k}(u+z) d \lambda(z) \leq\left(\frac{C_{2}}{\beta}\right)^{\nu / 2} \psi_{\nu, k}(u) .
$$

Case 2. distance $\left(u, \partial C_{\alpha}\right) \leq A$.

Observe finally that we are essentially dealing with two types of functions

$$
\psi=r^{n} \xi^{a}, r^{-a} \xi^{n},
$$

where the notations and $a, n \geq 0$ are as in (F.1.12) and that the estimates obtained must be uniform in $n$. Clearly also because of the symmetry about the axis of $C_{\alpha}$ of the above functions we may suppose that $u$ lies in the half of $C_{\alpha}$ that is closest to $\theta=0$. We can then substitute in the integrand the following two functions $\tilde{\psi}$ (that up to a multiplicative constant, dominate $\psi$ )

$$
\tilde{\psi}(z)=y^{a} x^{n}, \tilde{\psi}=x^{-a} y^{n}, \quad z=(x, y) \in C_{\alpha}=\left\{0<\theta<2 \theta_{0}\right\}
$$


and $\tilde{\psi}$ is assumed to be $\equiv 0$ outside $C_{\alpha}$.

For these new functions, and an appropiate choice of $R, \varepsilon$ and $A$ as above, the verification that we have to make in Case 2 reduces to

$$
\int_{|z| \geq \beta} \tilde{\psi}_{\nu, k}(u+z) d \lambda(z) \leq\left(\frac{C_{2}}{\beta}\right)^{\nu / 2} \tilde{\psi}_{\nu, k}((x, A)),
$$

for $u=(x, y),|u| \gg 1,0<y<A$. Finally if $0<\operatorname{Arg} u<\theta_{0}$, i.e. if $u$ lies in the half of $C_{\alpha}$ closest to the $x$-axis, it is easy to see that it suffices to make the above verifications with a modified $\tilde{\psi}$ given by

$$
\tilde{\psi}(z)= \begin{cases}y^{a} x^{n}, x^{-a} y^{n}, & z=(x, y), y>0, x>1 \\ 0, & \text { otherwise }\end{cases}
$$

Four inequalities have to be verified (uniformly in $n$ ) and I can see no other way than to just compute. Or rather let the reader compute for himself. At this point life can be made considerably simpler if we impose the following stronger condition on $\lambda$

$$
d \lambda(z) \leq C_{N}(1+|x|)^{-N}(1+|y|)^{-N} d z \quad \text { for all } N \geq 1 .
$$

This condition if applied to (F.1.14) "splits" with respect to the two variables $x$ and $y$ and the calculations simplify since they now reduce to the calculation of 1 dimensional integrals. Given that for all our applications the above stronger condition on $\lambda$ actually holds the verification under this stronger condition is "good enough". The details will be left to the interested reader.

\section{F.2. An alternative approach and higher dimensions.}

For the dimension $n=2$ the method that I developed in Section F.1 is unduly complicated. Indeed in the case $n=2$ it is much easier (and also throws additional light to the problem) to procede differently.

I shall briefly outline here this alternative method. We shall only examine what happens close to the boundary $\partial C_{\alpha}$, because for $\theta$ away from $\pm \theta_{0}$ everything is much easier. We shall therefore use the formula (F.1.1). If we denote by $\operatorname{Hess} F=\left(a_{i j}\right)_{i, j=1,2}$ the coefficients of that Hessian it is very easy to verify that for any $\varepsilon_{0}>0$ there exist $k_{0}, \nu_{0}, \theta_{0}$ (all depending on $\varepsilon_{0}$ ) such that

$$
\left(1+\varepsilon_{0}\right) a_{11} a_{22} \geq a_{12}^{2}, \quad \nu \geq \nu_{0}, k \geq k_{0}, 0 \leq \theta \leq \theta_{0} .
$$


The key to this alternative method is to show that under appropiate conditions on the measure $\mu(z)$, we can "make up" for the factor $\left(1+\varepsilon_{0}\right)$ and guarantee that the matrix $B=\left(b_{i j}\right)_{i, j=1,2}$

$$
\begin{aligned}
& b_{11}=\int a_{11}(z) \cos ^{2} \theta d \mu(z), \\
& b_{12}=\int a_{12}(z) \cos \theta \sin \theta d \mu(z), \\
& b_{22}=\int a_{22}(z) \sin ^{2} \theta d \mu(z),
\end{aligned}
$$

satisfies $b_{11} b_{22} \geq b_{12}^{2}$. The matrix $B$ is therefore positive definite and our proposition follows.

The details of the above method are easy to carry out. At any rate they are much easier than what was done in Section F.1. The reason why I presented the proof for $n=2$ in Section F.1 as I did was because the method of Section F.1 generalizes in a more or less obvious way (although the computations are somewhat tedious to carry out) to higher dimensions. I shall not write the proof down for $n \geq 3$ here. Indeed in a future publication the whole problem will be reexamined from a more general point of view.

\section{F.3. A final remark.}

The proofs given in this section of the appendix are very technical, to say the least. All this work seems to be incompressible if we wish to consider convolution operators with an arbitrary Gs-measure $\mu \in \mathbb{P}(G)$ as in Theorem B. If however we only wish to develop the necessary tools for the lower estimate of $\mathrm{A}_{2}$ ) then a completely different approach (that is more sophisticated and deep but technically much easier) can be used.

This approach will be developed at great lenght elsewhere I shall give however here the basic principles. It relies on the following two facts:

1) There exists $u \geq 0$ some non zero function on $X$ that is continuous, vanishes outside $C \times K$, and satisfies $D u=0$ in $C \times K$.

The existence of such a positive "harmonic" function relies on non trivial ideas from potential theory (A. Ancona [32] and L. Carleson [33] are the key references) which we have to adapt in our context. 
2) A function $u$ that satisfies the above conditions is automatically unbounded and of polynomial growth.

The proof of 2) is "lighter" than that of 1) but does rely on a scaled Harnack principle which, for large balls, can only be obtained by the Moser iterative process ( $c f$. [34]). At any rate all the details will eventually be presented in a separate paper.

\section{References.}

[1] Varopoulos, N. Th., Saloff-Coste, L., Coulhon, Th., Analysis and geometry on groups. Cambridge Univ. Press, 1992.

[2] Hörmander, L., Hypoelliptic second order equations. Acta Math. 119 (1967), 147-171.

[3] Varopoulos, N. Th., Thèorie de Hardy-Littlewood sur les groupes de Lie. C. R. Acad. Sci. Paris, Serie I 316 (1993), 999-1003.

[4] Bony, J. M., Principe du maximum, inégalité de Harnack et unicité du problème de Cauchy pour les opérateurs elliptiques dégénérés. Ann. Inst. Fourier 19 (1969), 277-304.

[5] Jacobson, N., Lie algebras. Interscience, 1962.

[6] Varadarajan, V. S., Lie groups, Lie algebras and their representations. Prentice-Hall, 1984.

[7] Chevalley, C., Théorie des groupes de Lie, tomes II \& III. Hermann, 1955 .

[8] Jacobson, N., Basic algebra, vol. I \& II. W. H. Freeman \& Comp. 1989.

[9] Helgason, S., Differential geometry, Lie groups and symmetric spaces, vol. 80. Academic Press, 1978. Groups and geometric analysis, vol. 113. Academic Press, 1984.

[10] Onischik, A. L., Vinberg, E. B., Lie groups and algebraic groups. Springer-Verlag, 1988.

[11] Guivarc'h, Y., Croissance polynomiale et périodes des fonctions harmoniques. Bull. Soc. Math. France 101 (1973), 333-379.

[12] Alexopoulos, G., An application of homogenization theory to harmonic analysis: Harnack inequalities and Riesz transforms on Lie groups of polynomial growth. Can. J. Math. 44 (1992), 691-727.

[13] Varopoulos, N. Th., Diffusion on Lie groups (I) and (II). Can. J. Math. 46 (1994), 438-448 and 1073-1093.

[14] Knapp, A., Representation theory of semisimple groups. Princeton Univ. Press, 1986. 
[15] Varopoulos, N. Th., Hardy-Littlewood theory on unimodular groups. Ann. Inst. H. Poincaré 31 (1995), 669-688.

[16] Reiter, H., Classical harmonic analysis and locally compact groups. Oxford Math. Monograph, 1968.

[17] Moser, J., On the volume elements on a manifold. Trans. Amer. Math. Soc. 120 (1965), 286-294.

[18] Gromov, M., Asymptotic invariance of infinite groups. I.H.E.S.-M-92-8.

[19] Varopoulos, N. Th., Small time Gaussian estimate of Heat diffusion kernels (I) and (II). Bull Sc. Math. 113 (1989), 253-277. J. Funct. Anal. 93 (1990), 1-33.

[20] Hebisch, W., Estimates on the semigroups generated by left invariant operators on Lie groups. J. für die reine und angew. Math. 423 (1992), $1-45$.

[21] Vauthier, J., Bull. Sci. Math., 2e série 103 (1979), 129-177.

[22] Ibragimov, I. A., Linnik, Yu. V., Independent and stationary sequences of random variables. Wolters-Noordhoff, 1971.

[23] Hörmander, L., The analysis of linear partial differential operators, vol. III. Springer-Verlag, 1985.

[24] Bers, L., John, F., Schechter, M., Partial differential equations. Lectures in appl. Math., vol. III. Interscience, AMS, 1974.

[25] Bourbaki, N., Eléments de mathématiques, Livre VI, Intégration. Hermann, 1963.

[26] Warner, G., Harmonic analysis on semisimple Lie groups, vol. I. Springer-Verlag, 1972.

[27] Burkholder, D. L., Distribution function inequalities for martingales. Ann. Prob. 1 (1973), 19-42.

[28] Mc Kean, H. P., Jr., Stochastic integrals. Academic Press, 1969.

[29] Stroock, D. W., Varadhan, S. R. S., Multidimensional diffusion processes. Springer-Verlag, 1979.

[30] Varadhan, S. R. S., Larges deviations and applications. SIAM, 1984.

[31] Schaefer, H. H., Banach lattices and positive operators. Springer-Verlag, 1975.

[32] Ancona, A., Principe de Harnack à la frontière et théorème de Fatou pour un operateur elliptique dans un domaine lipchitzien. Ann. Inst. Fourier 28-4 (1978), 162-213.

[33] Carleson, L., On the existence of boundary values for harmonic functions of several variables. Ark für Math. 4, (1962).

[34] Varopoulos, N. Th., Fonctions harmoniques sur les groupes de Lie. C. R. Acad. Sci. Paris 304(I) (1987), 519-521.

[35] Wallach, N. R., Real reductive groups, vol. I. Academic Press, 1988. 
[36] Feller, W., An introduction to probability theory and its applications, vol. I \& II. Wiley, 1968.

[37] Varopoulos, N. Th., Wiener-Hopf theory and non-unimodular groups. J. Funct. Anal. 120(2) (1994), 467-483.

[38] Varopoulos, N. Th., Potential theory of non-unimodular groups: "Harmonic analisys and discrete potential theory". Plenum Press, 1992.

[39] Bensoussan, A., Lions, J. L., Papanicolaou, G., Asymptotic analysis for periodic structures. North-Holland, 1978.

[40] Varopoulos, N. Th., Mustapha, S., Forth coming book. Cambridge University Press.

[41] Bougerol, Ph., Théorème central limit local sur certain groupe de Lie. Ann. Sci. Ec. Norm. Sup. $4^{\mathrm{e}}$ sér. 14 (1981), 403-432.

[42] Steenrod, N., The topology of fiber bundles. Princeton University Press, 1951.

[43] Varopoulos, N. Th., Distance distortion on Lie Groups. Institute MittagLeffler Report 31, 1995/96.

Recibido: 15 de junio de 1.995

Revisado: 25 de julio de 1.995

Nick Th. Varopoulos I.U.F. and

Departement de Mathématiques

Université de Paris VI

75005 Paris, FRANCE 